\title{
Neue Methoden zur Fluorierung von Verbindungen früher Übergangsmetalle
}

\author{
Dissertation \\ zur Erlangung des Doktorgrades \\ der Mathematisch-Naturwissenschaftlichen Fakultäten \\ der Georg-August-Universität zu Göttingen
}

\author{
vorgelegt von \\ Diplom-Chemiker \\ Mark Schormann \\ aus Bremen
}

Göttingen 2000 
D 7

Referent: $\quad$ Prof. Dr. Dr. h. c. mult. H. W. Roesky

Korreferent: $\quad$ Prof. Dr. L. Fitjer

Tag der mündlichen Prüfung: 30.10.2000 


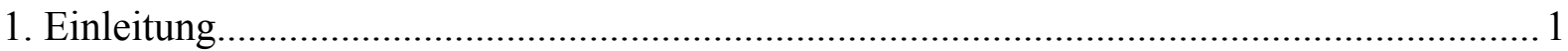

1.1. Ausgewählte Beispiele angewandter Fluorchemie …................................................. 1

1.2. Eigenschaften organometallischer Fluorokomplexe ................................................ 1

1.3. Synthesen organometallischer Fluorokomplexe ......................................................... 2

1.4. Fluorokomplexe als Katalysatoren für die Olefinpolymerisation................................ 4

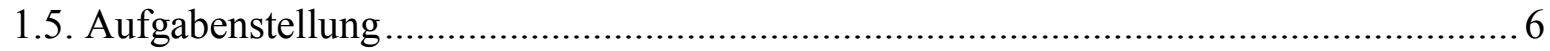

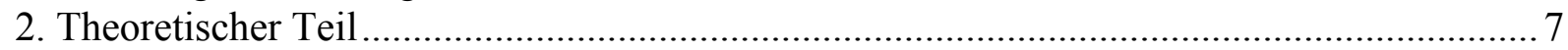

2.1. Synthese von Bis(trimethylsilyl)methyl-Komplexen von Niob, Tantal und Titan ......... 7

2.1.1. Der Bis(trimethylsilyl)methyl-Rest als vielseitiger Ligand in der

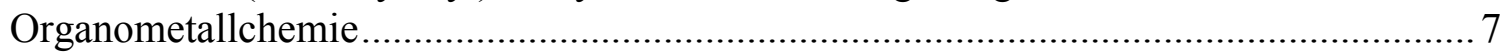

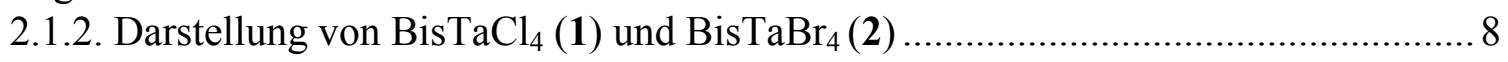

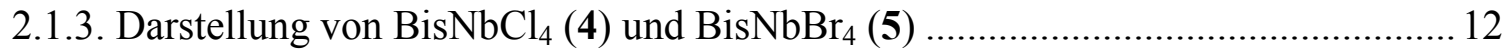

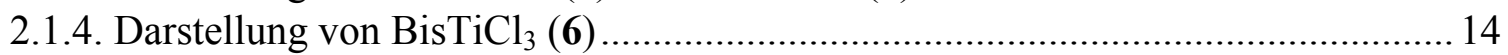

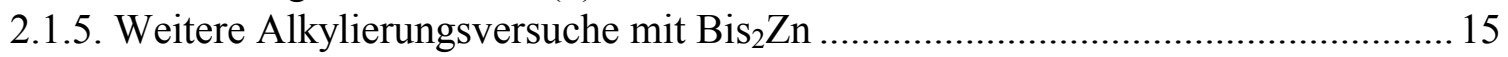

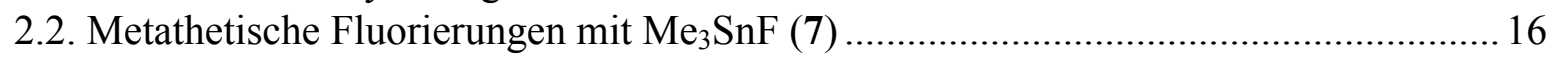

2.2.1. Diskussion der Einkristallröntgenstrukturanalyse von $\mathrm{Ns}_{3} \mathrm{SnCl}(\mathbf{8}) \ldots \ldots \ldots \ldots \ldots \ldots \ldots . . . . . . . . .17$

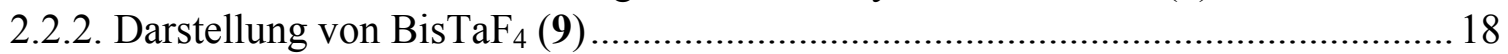

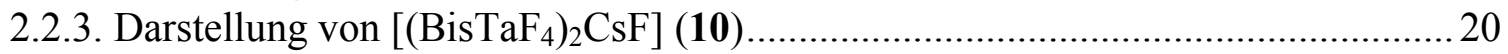

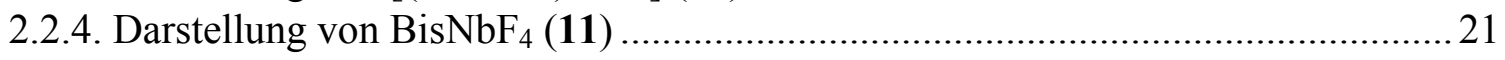

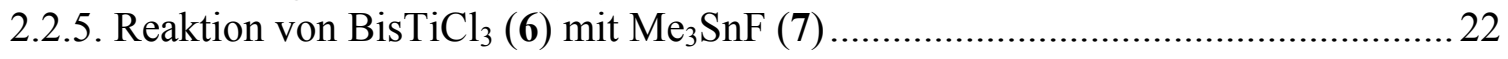

2.2.6. Versuch der Fluorierung von $\mathrm{Silox}_{3} \mathrm{TaCl}_{2}\left(\mathrm{Silox}=(t-\mathrm{BuO})_{3} \mathrm{SiO}-\right)$ mit $\mathrm{Me}_{3} \mathrm{SnF}(7)$

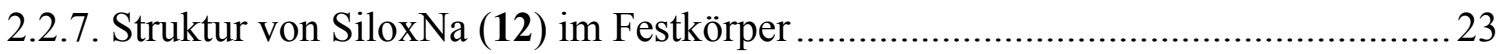

2.2.8. Vergleich der NMR-Daten der neuen Bis-substituierten Verbindungen................ 25

2.3. Einsatz der neuen Fluorierungsmittel $\mathrm{Ph}_{2} \mathrm{PbF}_{2}$ (13) und $\mathrm{Ph}_{3} \mathrm{BiF}_{2}(\mathbf{1 4})$............................ 26

2.3.1. Vergleich der Eigenschaften von $\mathrm{Ph}_{2} \mathrm{PbF}_{2}$ (13) und $\mathrm{Ph}_{3} \mathrm{BiF}_{2}$ (14) mit $\mathrm{Me}_{3} \mathrm{SnF}$ (9)26

2.3.2. Darstellung von $\mathrm{Cp}^{*} \mathrm{TiF}_{3}, \mathrm{Cp}_{2} \mathrm{ZrF}_{2}$ undCp $\mathrm{P}_{2} \mathrm{HfF}_{2}$ unter Verwendung von 13 und 1428

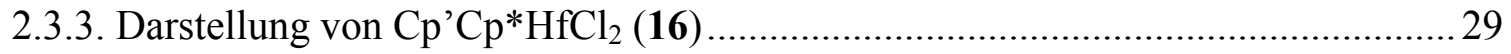

2.3.4. Versuch der Monofluorierung von $\mathrm{Cp}^{*} \mathrm{Cp}^{\prime} \mathrm{MCl}_{2}(\mathrm{M}=\mathrm{Zr}$, Hf) mit 13 ..................29

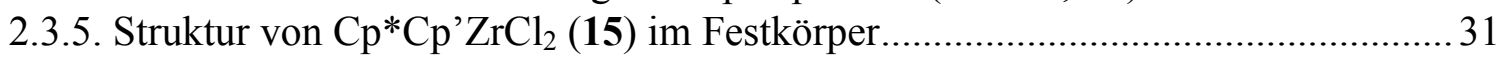

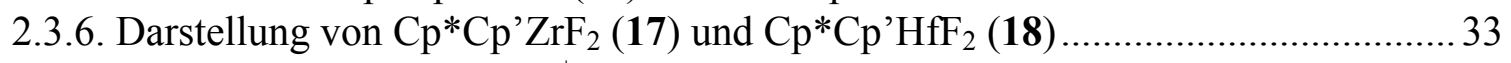

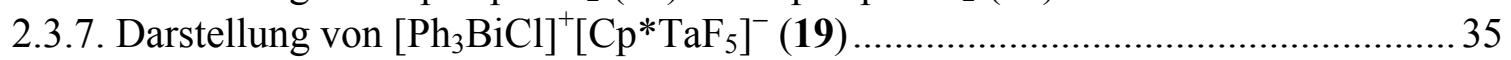

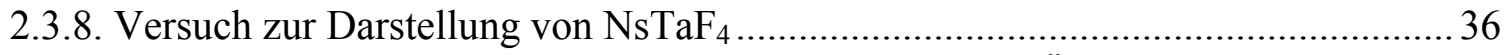

2.3.9. Versuche zur Darstellung von Fluorokomplexen später Übergangsmetalle............ 36

2.3.10. Abschließender Vergleich der Fluorierungsmittel $\mathrm{Me}_{3} \mathrm{SnF}$ (7), $\mathrm{Ph}_{2} \mathrm{PbF}_{2}$ (13) und

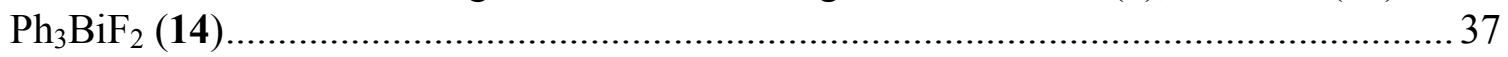

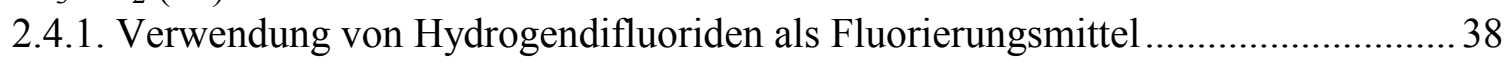

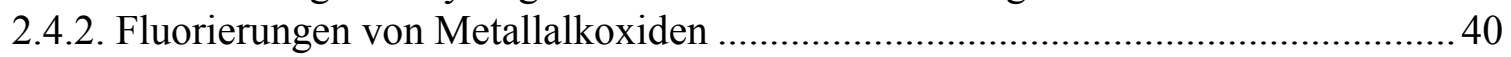

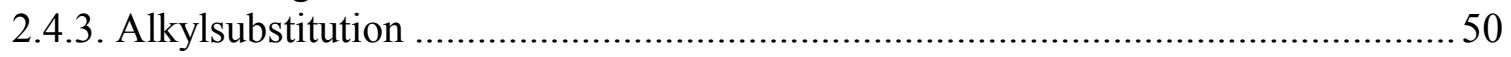

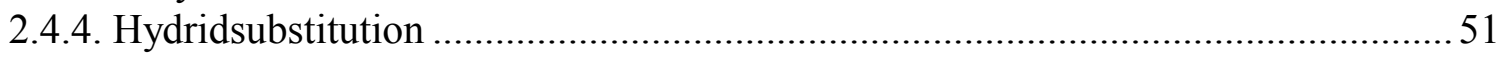

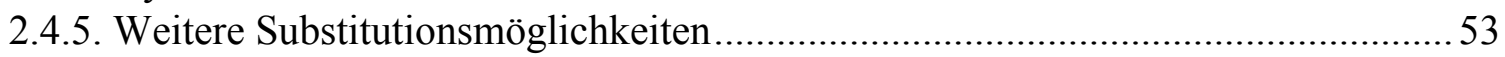

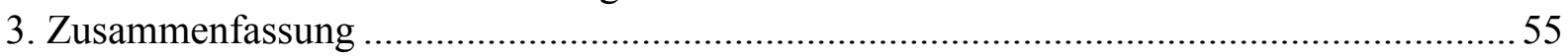

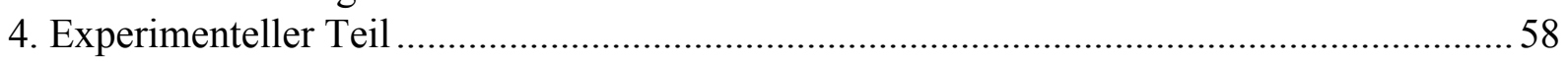

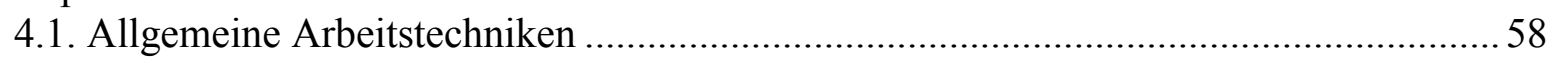

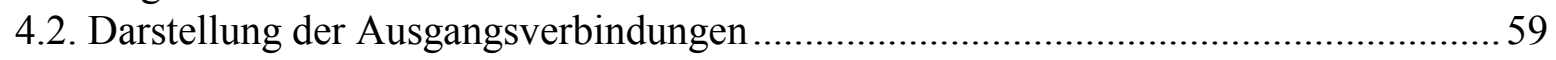

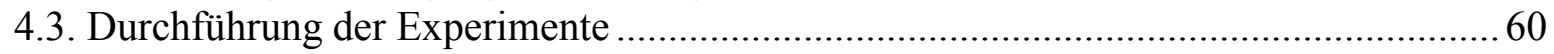

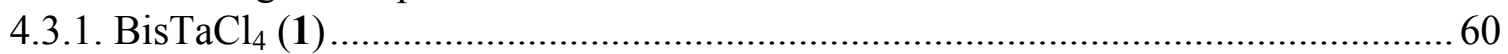




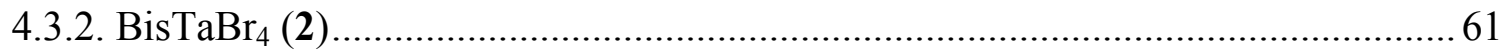

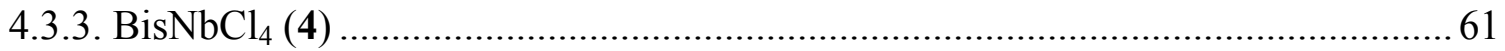

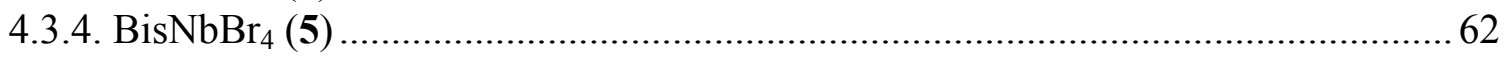

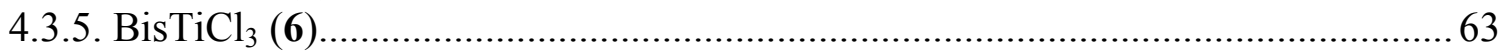

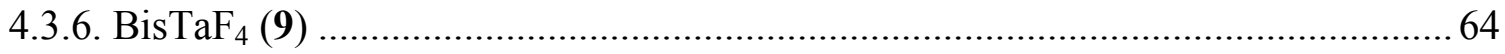

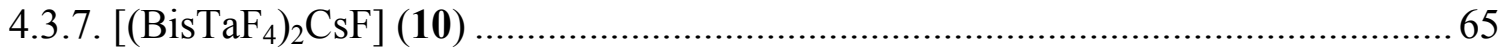

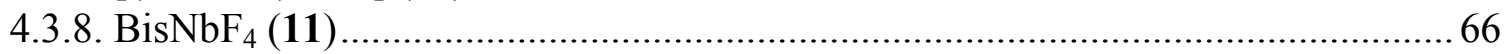

4.3.9. Allgemeine Vorschrift zur Fluorierung mit $\mathrm{Ph}_{2} \mathrm{PbF}_{2}$ (13) und $\mathrm{Ph}_{3} \mathrm{BiF}_{2}($ 14) ......... 67

4.3.10. Darstellung von $\mathrm{Cp}^{*} \mathrm{TiF}_{3}, \mathrm{Cp}_{2} \mathrm{ZrF}_{2}$ undCp $\mathrm{CfFF}_{2}$ jeweils mit $\mathrm{Ph}_{2} \mathrm{PbF}_{2}$ (13) und

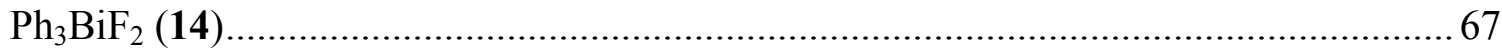

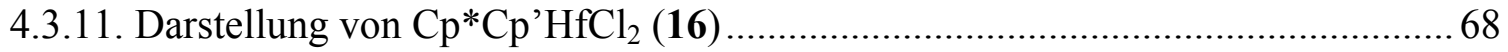

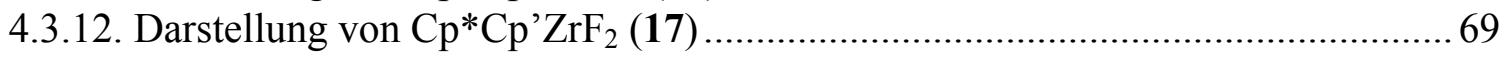

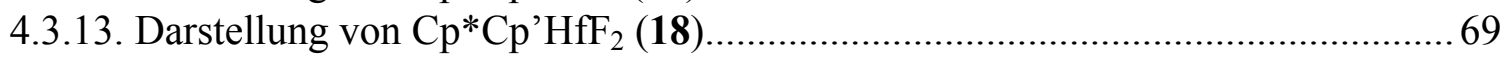

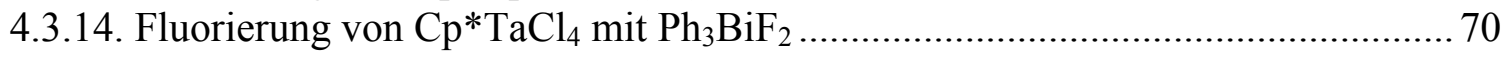

4.3.15. Darstellung von $\mu_{3}$-Fluoro- $\mu_{3}$-oxo-tris( $\mu$-isopropoxi)-hexakis(isopropoxi)-tri-

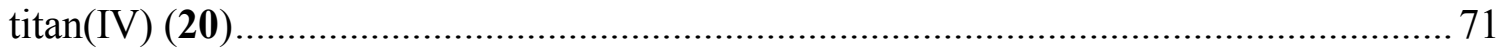

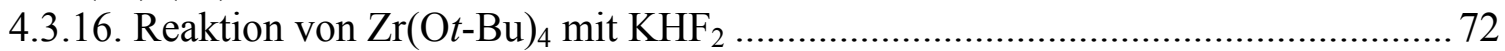

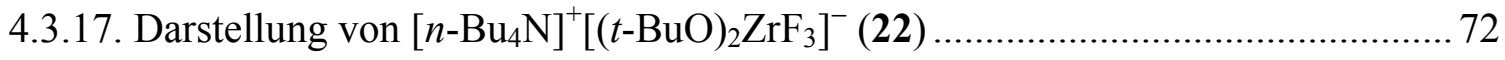

4.3.18. Darstellung von $\operatorname{Bis}\left(\mu_{3}\right.$-oxo)-hexakis $(\mu$-ethoxi)-octakis $(\mu$-oxo)tetradekakis(ethoxi)-octa-tantal(V) (23) ................................................................... 73

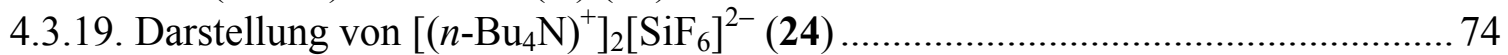

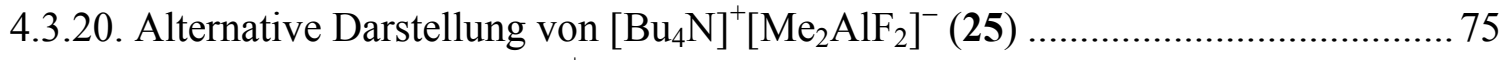

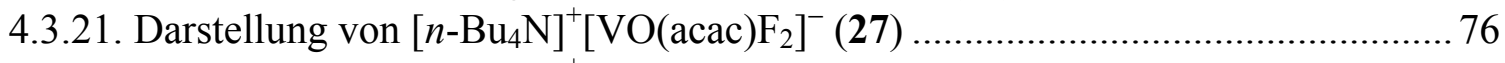

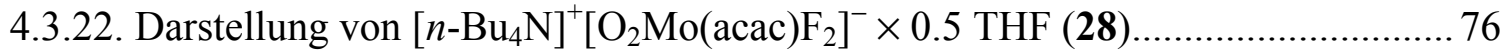

4.4. Behandlung und Entsorgung der Abfälle................................................................ 77

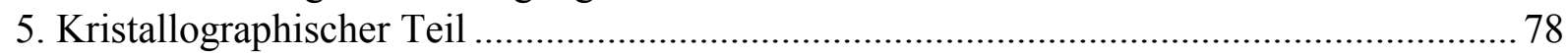

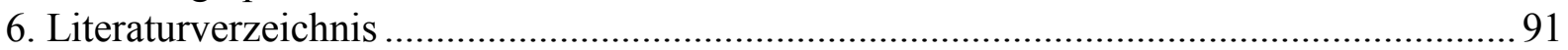


Abb.

acac

Ber.

Bis

$n$-Bu

$t$-Bu

$\mathrm{Cp}$

$\mathrm{Cp}^{*}$

Cp'

Cp',

EI

endst.

Et

exc.

FAB

Gef.

$\mathrm{h}$

$\mathrm{Hz}$

IR

$J$

$\mathrm{kJ}$

M

MAO

$\mathrm{Me}$

$\min$.

$\mathrm{MHz}$

$\mathrm{m} / \mathrm{z}$

NBA

NBS

NCS

NMR
Abbildung

Acetylacetonat

Berechnet

Bis(trimethylsilyl)methyl

n-Butyl

tert-Butyl

Cyclopentadienyl

Pentamethylcyclopentadienyl

Trimethylsilylcyclopentadienyl

Bis(trimethylsilyl)cyclopentadienyl

Elektronenstoßionisation

endständig

Ethyl

Überschuß

Fast Atom Bombardment

Gefunden

Stunde(n)

Hertz

Infrarot

Kopplungskonstante

Kilojoule

Molekülion

Methylaluminoxan

Methyl

Minute

Megahertz

Masse-Ladungs-Verhältnis

3-Nitrobenzylalkohol

N-Bromsuccinimid

N-Chlorsuccinimid

kernmagnetische Resonanz 
$\mathrm{Np}$

Ns

$\mathrm{O} t-\mathrm{Bu}$

OEt

$\mathrm{O} i$-Pr

ppm

PS

$\mathrm{R}$

Silox

TBADF

TBAF

THF

TMS

Triflat

verb.

$\delta$

$\mu$

$\widetilde{v}$
Neopentyl

Trimethylsilylmethyl

tert-Butoxi(d)

Ethoxi(d)

iso-Propoxi(d)

parts per million

Polystyrol

organischer Rest

$t-\mathrm{Bu}_{3} \mathrm{SiO}^{-}$

Tetra- $n$-butylammoniumhydrogendifluorid

Tetra-n-butylammoniumfluorid

Tetrahydrofuran

Tetramethylsilan

Trifluormethylsulfonat

verbrückend

chemische Verschiebung

verbrückend

Wellenzahl 


\section{Einleitung}

\subsection{Ausgewählte Beispiele angewandter Fluorchemie}

Die erste Fluorverbindung, die in der Literatur Erwähnung findet, ist der Flußspat. Im Jahre 1529 berichtet der Alchimist Agricola über die Verwendung von Flußspat $\left(\mathrm{CaF}_{2}\right)$ als Flußmittel beim Schmelzen von Erzen. Daher hat das Element Fluor (= Fluß) auch seinen Namen bekommen. Bis zum heutigen Tage sind natürlich viele Anwendungen hinzugekommen. Chlorfluorkohlenwasserstoffe (CFK oder FCKW) wurden lange Zeit als Kühl- und Treibmittel eingesetzt. Aufgrund ihrer ozonschädigenden Eigenschaft wird jedoch ihre Anwendung gemieden [1]. Zur Anreicherung von spaltfähigem Material für die Herstellung von Kernbrennstäben benötigt man Uranhexafluorid, das man aus $\mathrm{UO}_{2}, \mathrm{HF}$ und $\mathrm{F}_{2}$ gewinnen kann [2]. Fluorelastomere, von denen Teflon am häufigsten Verwendung findet, zeichnen sich sowohl durch ihre chemische Inertheit als auch durch eine hohe Temperaturbeständigkeit aus [3]. Flüssige perfluorierte Kohlenwasserstoffe sind in der Lage, große Mengen Sauerstoff zu speichern. In Versuchen an lebenden Ratten konnte gezeigt werden, daß die Tiere diese Flüssigkeiten atmen können. Daraufhin wurde große Anstrengung unternommen, aus diesen Systemen Blutersatzstoffe zu entwickeln [4].

\subsection{Eigenschaften organometallischer Fluorokomplexe}

Die metallorganischen Fluoride unterscheiden sich in ihren Eigenschaften bezüglich Oligomerisierungsgrad, Löslichkeit und Flüchtigkeit stark von den anderen Halogeniden. Aus diesem Grund besteht ein großes Interesse an der Synthese von Verbindungen dieser Substanzklasse [5-7]. Es ist interessant, organometallische Fluoride in katalytischen Prozessen oder zur Synthese einzusetzen. In diesem Zusammenhang wurden Olefinpolymerisationen [8], enantioselektive Hydrosilylierungen prochiraler Amine [9], Reduktionen von perfluorierten Kohlenwasserstoffen [10], Transaminierungen von organometallischen Fluoriden der 4. Gruppe [11], Synthese von Verbindungen mit Hauptgruppen- Übergangsmetallbindungen $\quad[12,13], \quad$ intramolekulare $\quad \mathrm{C}$-F- 
Bindungsaktivierung $\quad[14,15], \quad$ C-H-Bindungsaktivierung $\quad[16]$, Trifluormethanidübertragungsgleichgewichte [17], hochreaktive Lewissäuren mit Anwendungen in der asymmetrischen Katalyse [18,19] und Wirt-Gast- Chemie [12] untersucht.

Die Synthese der Ausgangsverbindungen ist oft mit erheblichem präparativem Aufwand verbunden, weil für die Darstellung der organometallischen Fluorokomplexe keine einheitliche Synthesemethode existiert und diese erst individuell entwickelt werden muß.

Die strukturellen Unterschiede zwischen Chloro- und Fluorokomplexen lassen sich mit der hohen Elektronendichte und dem damit verbundenen starken $\pi$-Donorvermögen erklären [20]. Daher neigen Fluorokomplexe früher Übergangsmetalle zur Oligomerisierung. Dieses wird anhand der Komplexe $\left(\mathrm{Cp}^{*} \mathrm{TiF}_{3}\right)_{2}$ [21], $\left(\mathrm{Cp} * \mathrm{ZrF}_{3}\right)_{4}$ [21] und $\left(\mathrm{Cp}^{*} \mathrm{TaF}_{4}\right)_{2}$ [22, 23] deutlich.

Die hohe Energie der Metall-Fluor-Bindung erschwert die Aktivierung für weitere Reaktionen, wodurch sich auch die höhere thermische Belastbarkeit von Fluoro- gegenüber Chlorokomplexen erklären läßt.

\subsection{Synthesen organometallischer Fluorokomplexe}

Eine Vielzahl von Chloriden und Bromiden organometallischer Übergangsmetallkomplexe sind bereits dargestellt und in der homogenen Katalyse eingesetzt worden [24]. Allerdings wurden nur wenige Fluoroverbindungen synthetisiert und deren Reaktivität und katalytische Aktivität untersucht, weil diese Verbindungsklasse schlechter zugänglich ist. Es ist fast immer unmöglich, die reinen Metallfluoride zu derivatisieren, weil sie eine polymere Struktur aufweisen. Aus diesem Grund müssen die Fluoratome in einem späteren Schritt eingebaut werden. Die Wahl des Fluorierungsmittels ist dabei von entscheidender Bedeutung [25].

Die Fluorierung kann durch Metathese der entsprechenden Chlorokomplexe mit Hilfe von ionischen Fluoridquellen wie NaF oder AgF geschehen. Aufgrund von Komplexierungen des entstehenden Organometallfluorids durch freies Fluorid läßt sich das gewünschte Produkt nicht rein oder nur in geringer Ausbeute isolieren [26-28]. Als Abfangprodukt wurde der Wirt-Gast Komplex $\left[\mathrm{PPh}_{4}\right]^{+}\left[\mathrm{Cp}_{4} \mathrm{~F}_{14} \mathrm{NaTi}_{4}\right]^{-}$strukturell aufgeklärt [29]. Die Methode der Wahl zur Synthese von Fluorokomplexen der 4. - 6. Gruppe ist der in der Arbeitsgruppe Roesky 
entwickelte Chlorid-Fluorid-Austausch durch $\mathrm{Me}_{3} \mathrm{SnF}$ [21]. Die Verbindung ist ein luft-, wasser- und temperaturbeständiger, farbloser Feststoff, der in großer Menge hergestellt werden kann. Das bei der Reaktion entstehende $\mathrm{Me}_{3} \mathrm{SnCl}$ kann unter Vakuum abkondensiert und mit Hilfe von $\mathrm{NaF}$ kann $\mathrm{Me}_{3} \mathrm{SnF}$ zurückerhalten werden, so daß man formal nur $\mathrm{NaF}$ für die Fluorierung einsetzt.

$$
\begin{aligned}
& \mathrm{Cp}^{*}{ }_{\mathrm{m}} \mathrm{MCl}_{\mathrm{n}}+\mathrm{n} \mathrm{Me} \mathrm{Me}_{3} \mathrm{SnF} \longrightarrow \mathrm{Cp}^{*}{ }_{\mathrm{m}} \mathrm{MF}_{\mathrm{n}}+\mathrm{n} \mathrm{Me}_{3} \mathrm{SnCl} \\
& \mathrm{Me}_{3} \mathrm{SnCl}+\mathrm{NaF} \longrightarrow \mathrm{Me}_{3} \mathrm{SnF}+\mathrm{NaCl} \\
& \hline \mathrm{Cp}^{*}{ }_{\mathrm{m}} \mathrm{MCl}_{\mathrm{n}}+\mathrm{n} \mathrm{NaF} \longrightarrow \mathrm{Cp}^{*}{ }_{\mathrm{m}} \mathrm{MF}_{\mathrm{n}}+\mathrm{n} \mathrm{NaCl}
\end{aligned}
$$

Sauerstoff $[21,30,31]$ und Stickstoff [11] enthaltende Liganden werden von $\mathrm{Me}_{3} \mathrm{SnF}$ toleriert. Weitere Synthesewege werden durch Metathese von Metallhydriden [32] und Alkylderivaten [33, 34] eröffnet.
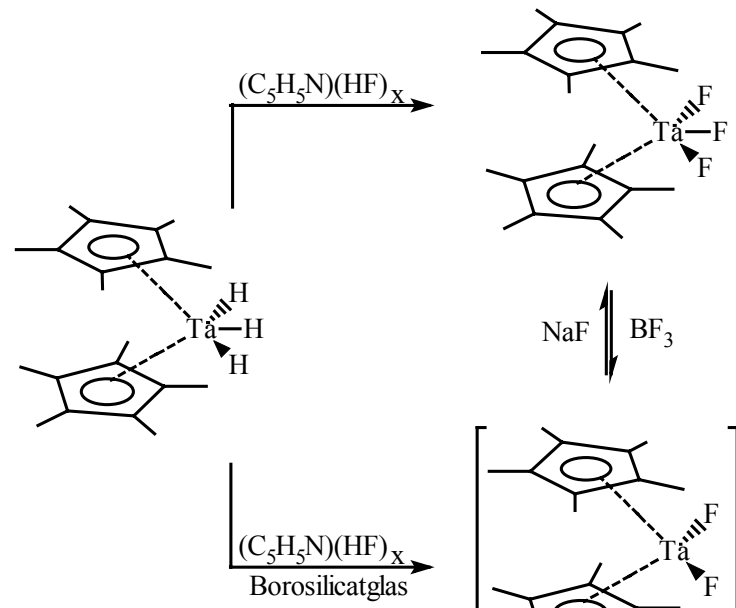

Des weiteren finden HF-Derivate als Fluorierungsmittel Verwendung. Dabei macht man sich das Prinzip zunutze, daß die vom Metallzentrum abzuspaltende Gruppe unter Protonierung aus dem Gleichgewicht verdrängt wird. Da eine Reaktionsführung mit reinem HF leicht zur Abspaltung des organischen Liganden führt, wird die Reaktivität des HF durch Koordination an eine Stickstoffbase (Collidin [33] oder Pyridin [35]) herabgesetzt. Auf diesem Weg konnte $\mathrm{Cp}^{*} \mathrm{ZrF}_{3}$ aus $\mathrm{Cp}^{*} \mathrm{Zr}\left(\mathrm{CH}_{2} \mathrm{Ph}\right)_{3}$ und Collidin(HF $)_{2}$ unter Freisetzung von Toluol synthetisiert werden [33]. Weitere für diesen Syntheseweg geeignete Ausgangsverbindungen sind organometallische Hydride. Der $18-\mathrm{e}^{-}-$ Komplex $\mathrm{Cp}_{2}{ }_{2} \mathrm{TaH}_{3}$ konnte mittels Pyridin(HF) in den Fluorokomplex $\mathrm{Cp}_{2}{ }_{2} \mathrm{TaF}_{3}$ überführt werden [36]. Die Bildung von $[\mathrm{Coll}-\mathrm{H}]^{+}\left[\mathrm{Cp}^{*}{ }_{2} \mathrm{Zr}_{2} \mathrm{~F}_{7}\right]^{-}$und $\left[\mathrm{Et}_{3} \mathrm{~N}-\mathrm{H}\right]^{+}\left[\mathrm{Cp}^{*} \mathrm{TaF}_{5}\right]^{-}$zeigt, daß eine Koordination vom freien Amin am Produkt nicht immer vermieden werden kann. Ein weiterer unerwünschter Effekt ist der Angriff des Fluorierungsmittels auf das Borosilikatglas. 
Dabei entsteht intermediär Tetrafluoroborsäure, die die Bildung von $\left[\mathrm{Cp}_{2}^{*} \mathrm{TaF}_{2}\right]^{+}\left[\mathrm{BF}_{4}\right]^{-}$ ermöglicht.

\subsection{Fluorokomplexe als Katalysatoren für die Olefinpolymerisation}

Einhergehend mit der Entdeckung neuer Fluorierungsmittel und der Entwicklung alternativer Synthesestrategien wurde die Voraussetzung für den Einsatz fluorhaltiger Komplexe als Katalysatoren in der Olefinpolymerisation geschaffen. Von Roesky und Kaminsky et al. wurden entsprechende Versuche zur Polymerisation von Ethen und Styrol mit Organotitan- und Organozirconium-Fluoriden in Kombination mit MAO (Methylaluminoxan) als Katalysator durchgeführt [8]. Auf diesem Weg konnte aus Styrol ein syndiotaktisches Polymer erhalten werden, ohne einen chiralen Katalysator einzusetzen. Ein Vergleich der Aktivitäten der Fluoro- mit den Chlorokomplexen (Tabelle 1) zeigt, daß die Fluoride generell eine höhere Aktivität aufweisen und bereits bei kleinerem Katalysator-CokatalysatorVerhältnis ihre maximale Aktivität erreichen. Dabei weist das Polymerisat einen höheren Schmelzpunkt auf, wenn Fluoride als Katalysatoren eingesetzt wurden. Die Substitution des Cp-Rings hat einen starken Einfluß auf die Aktivität des Katalysators. Die höchste Aktivität zeigt $\left(\mathrm{C}_{5} \mathrm{H}_{4} \mathrm{Me}\right) \mathrm{TiF}_{3}$ mit $14000 \mathrm{~kg}$ PS $\times(\text { Konz.(Kat) } \mathrm{h})^{-1}$.

Tabelle 1: Vergleich von Aktivität und Schmelzpunkt (Polymerisat) ausgewählter

Katalysatorkomplexe für die Darstellung von isotaktischem Polystyrol (PS)

\begin{tabular}{lcc}
\hline \multicolumn{1}{c}{ Katalysator } & Aktivität & Schmelzpunkt \\
\hline $\mathrm{CpTiCl}_{3}$ & 1100 & 258 \\
$\mathrm{CpTiF}_{3}$ & 3000 & 265 \\
$\mathrm{Cp} * \mathrm{TiCl}_{3}$ & 15 & 275 \\
$\mathrm{Cp}^{*} \mathrm{TiF}_{3}$ & 690 & 275 \\
$\mathrm{Cp}^{*} \mathrm{ZrCl}_{3}$ & 0.08 & 249 \\
$\mathrm{Cp}^{*} \mathrm{ZrF}_{3}$ & 0.9 & 248 \\
$\left(\mathrm{C}_{5} \mathrm{H}_{4} \mathrm{Me}\right)_{2} \mathrm{TiF}_{2}$ & 1.8 & 264 \\
$\left(\mathrm{C}_{5} \mathrm{H}_{4} \mathrm{Me}\right) \mathrm{TiF}_{3}$ & 14000 & 269 \\
\hline
\end{tabular}

Aktivität [kg PS (Konz.(Kat.) h) ${ }^{-1}$ ], Schmelzpunkt des syndiotaktischen PS [ $\left.{ }^{\circ} \mathrm{C}\right]$, Styrol Konzentration $=4.3 \mathrm{~mol} / \mathrm{l}$, Konz.(Kat.) $=6.25 \times 10^{-5} \mathrm{~mol} / \mathrm{l}, \mathrm{Al}: \mathrm{M}=300, \mathrm{~T}=50{ }^{\circ} \mathrm{C}$.

Besonderes Interesse gilt dem Zusammenwirken zwischen dem Kokatalysator (MAO) und dem Katalysator. Bislang ist es noch nicht gelungen, die Struktur des MAO aufzuklären [37 - 40]. Man vermutet aber, daß MAO das Metallzentrum alkyliert, ein Kation generiert und 
dieses mittels Koordination stabilisiert. Als katalytisch aktive Spezies wird ein kationischer Komplex vorgeschlagen: $\left[\mathrm{Cp}_{2} \mathrm{TiMe}\right]^{+}[\mathrm{MAO}-\mathrm{Me}]^{-}[41,42]$.

Um die katalytische Aktivität der metallorganischen Fluoride der 4. Gruppe in der Olefinpolymerisation genauer zu untersuchen, wurde selektiv eine Fluorfunktion durch eine Alkylgruppe mit Hilfe von Aluminiumtrimethyl substituiert [43]. Zu diesem Zweck wurde $\mathrm{Cp}^{*} \mathrm{ZrF}_{3}$ mit $\mathrm{AlMe}_{3}$ im molaren Verhältnis 1:1 zur Reaktion gebracht. Es erfolgte eine einfache Methylierung am Metallzentrum und das dabei entstandene Dimethylaluminiumfluorid koordinierte im Produkt als verbrückender Ligand zwischen zwei Metallzentren [43, 44]. Nach Umsetzung mit einem Überschuß $\mathrm{AlMe}_{3}$ resultierte ein Cluster, in dem alle Fluoratome substituiert wurden.

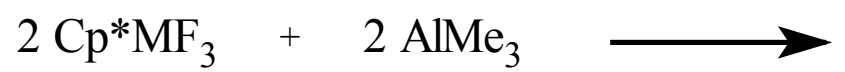

$(\mathrm{M}=\mathrm{Zr}, \mathrm{Hf})$

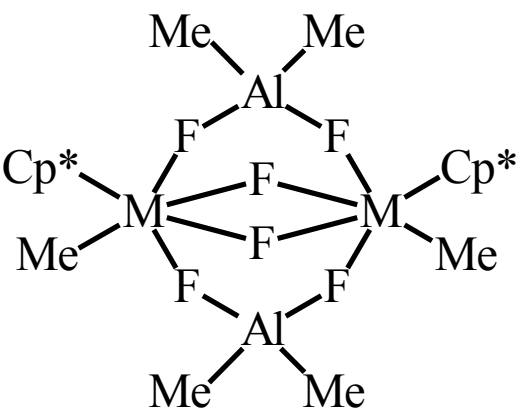

$3 \mathrm{Cp}^{*} \mathrm{MF}_{3}+15 \mathrm{AlMe}_{3} \longrightarrow\left[(\mathrm{Cp} * \mathrm{M})_{3} \mathrm{Al}_{6} \mathrm{Me}_{8}\left(\mu_{3}-\mathrm{CH}\right)_{5}\left(\mu-\mathrm{CH}_{2}\right)_{2}\right]$

+ $9 \mathrm{AlMe}_{2} \mathrm{~F}+12 \mathrm{CH}_{4}$ 


\subsection{Aufgabenstellung}

Ziel dieser Arbeit war es, unter Verwendung von $\mathrm{Me}_{3} \mathrm{SnF}$ als Fluorierungsmittel organometallische Fluorokomplexe zu synthetisieren, die eine Metall-Kohlenstoff- $\sigma$-Bindung aufweisen.

Im weiteren Verlauf sollte das Synthesepotential von $\mathrm{Ph}_{2} \mathrm{PbF}_{2}$ und $\mathrm{Ph}_{3} \mathrm{BiF}_{2}$ als Fluorierungsmittel untersucht werden. Hierbei stand die Frage im Vordergrund, ob auf diesem Weg Verbindungen, die nicht mit $\mathrm{Me}_{3} \mathrm{SnF}$ zugänglich sind, erhalten werden können.

In einem dritten Teil sollte die Bedeutung von Hydrogendifluoriden bei der Fluorierung von Alkoxid-, Alkyl-, Hydrid-, Acetat-, und Acetylacetonatverbindungen untersucht werden. 


\section{Theoretischer Teil}

\subsection{Synthese von Bis(trimethylsilyl)methyl-Komplexen von Niob, Tantal und Titan}

\subsubsection{Der Bis(trimethylsilyl)methyl-Rest als vielseitiger Ligand in der Organometallchemie}

Sowohl in der Chemie der Hauptgruppen als auch bei den Übergangsmetallen werden die elektronisch und sterisch stabilisierenden Eigenschaften des Bis-Liganden (Bis = $\left.\left(\mathrm{Me}_{3} \mathrm{Si}\right)_{2} \mathrm{CH}-\right)$ genutzt. So konnte eine Reihe von Hauptgruppenmetallverbindungen in niedrigen Oxidationsstufen dargestellt werden. $\mathrm{Zu}$ nennen sind hier exemplarisch $\left(\mathrm{Bis}_{2} \mathrm{Al}\right)_{2}$ [45], $(\mathrm{BisGe})_{6}[46],(\mathrm{BisSb})_{3}$ [47] und $(\mathrm{BisBi})_{4}$ [48], deren Kristallstrukturen bestimmt werden konnten. Weiter gelang die Stabilisierung von Verbindungen mit Doppelbindungen zwischen Hauptgruppenelementen der 15. Gruppe z.B. in $\left(t-\mathrm{Bu}_{3} \mathrm{C}_{6} \mathrm{H}_{2}\right) \mathrm{P}=\mathrm{AsBis}$ [49] und $\left(t-\mathrm{Bu}_{3} \mathrm{C}_{6} \mathrm{H}_{2}\right) \mathrm{As}=\mathrm{AsBis}$ [50]. Anhand der Stabilisierung von Komplexen der Seltenen Erden mit der Koordinationszahl drei wird der hohe sterische Anspruch des Bis-Liganden deutlich. In diesem Zusammenhang gelang Westerhausen et al. die Strukturaufklärung von $\mathrm{Bis}_{3} \mathrm{Y}$ [51].

Viele mit Bis-Liganden substituierte Übergangsmetallkomplexe lassen sich über eine Salzeliminierung aus BisLi und den entsprechenden Metallhalogeniden bzw. -alkoxiden darstellen. Auf diesem Weg lassen sich Zirconocenchloride [52] und Chromtrichlorid [53] substituieren. Bei Niob- und Tantalhalogeniden führt diese Synthesestrategie nicht zum Erfolg und daher waren entsprechende Verbindungen bisher nicht bekannt. Es erschien in diesem Zusammenhang sinnvoll zu überprüfen, ob derartige Verbindungen mit Hilfe des milderen Alkylierungsmittels $\mathrm{Bis}_{2} \mathrm{Zn}$ [54]zugänglich sind. Außerdem sollte das Synthesepotential des Zinkorganyls gegenüber weiteren Metallhalogeniden untersucht werden.

Der Bis-Ligand verleiht seinen Komplexen eine gute Löslichkeit in organischen Lösungsmitteln. Positive Einflüsse auf die Kristallisation sowie eine leichte Interpretierbarkeit der NMR-Spektren begünstigen die Charakterisierung. 


\subsubsection{Darstellung von BisTaCl 4 (1) und $\mathrm{BisTaBr}_{4}(2)$}

Für einen Bis-substituierten Tantalkomplex findet man nur ein Beispiel in der Literatur [55]. Ein Cp-substituiertes Ta(IV)-chlorid wurde mit BisLi alkyliert. Die Alkylierung von $\mathrm{TaCl}_{5}$ mit BisLi führt zu einem Produktgemisch, in dem BisH nachgewiesen werden kann. Im Gegensatz dazu lassen sich $\mathrm{BisTaCl}_{4}$ (1) und $\mathrm{BisTaBr}_{4}$ (2) aus dem Metallhalogenid und $\mathrm{Bis}_{2} \mathrm{Zn}$ darstellen. Setzt man $\mathrm{TaCl}_{5}$ und $\mathrm{Bis}_{2} \mathrm{Zn}$ im molaren Verhältnis $1: 1 \mathrm{um}$, läßt sich kein höher alkyliertes Produkt isolieren und man erhält ebenfalls 1 neben unverändertem $\mathrm{Bis}_{2} \mathrm{Zn}$. Außerdem wurde versucht, 1 mit $\mathrm{Me}_{2} \mathrm{Zn}$ zu methylieren. Dabei konnte kein einheitliches Produkt isoliert werden.

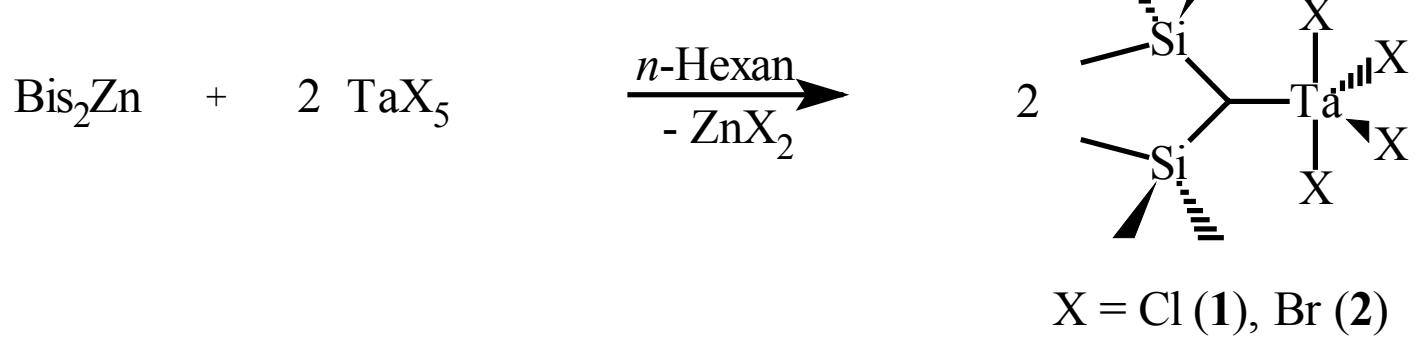

Die Verbindungen $\mathbf{1}$ und $\mathbf{2}$ bilden orangefarbige luft- und wasserempfindliche Kristalle, die in allen gängigen Lösungsmitteln sehr gut löslich sind. Verbindung 2 unterliegt der Zersetzung bei Raumtemperatur innerhalb von Stunden, was sich in einer Schwarzfärbung äußert. Die Chlorverbindung bleibt im Gegensatz dazu unverändert. Die Zusammensetzung und Konstitution von 1 und $\mathbf{2}$ wurde durch Elementaranalyse, NMR- und IR-Spektroskopie und Massenspektrometrie ermittelt. Die chemischen Verschiebungen der Protonen sind in Tabelle 8 aufgeführt.

Durch Umkristallisieren aus $n$-Hexan bei $-30{ }^{\circ} \mathrm{C}$ konnten für eine Einkristallröntgenstrukturanalyse geeignete Kristalle von $\mathbf{1}$ erhalten werden. Verbindung 1 kristallisiert in der triklinen Raumgruppe $P \overline{1}$ mit zwei Molekülen pro asymmetrischer Einheit.

Abbildung 1 zeigt die Struktur von 1 im Kristall. Charakteristische Bindungslängen und -winkel sind in Tabelle 2 aufgeführt. 


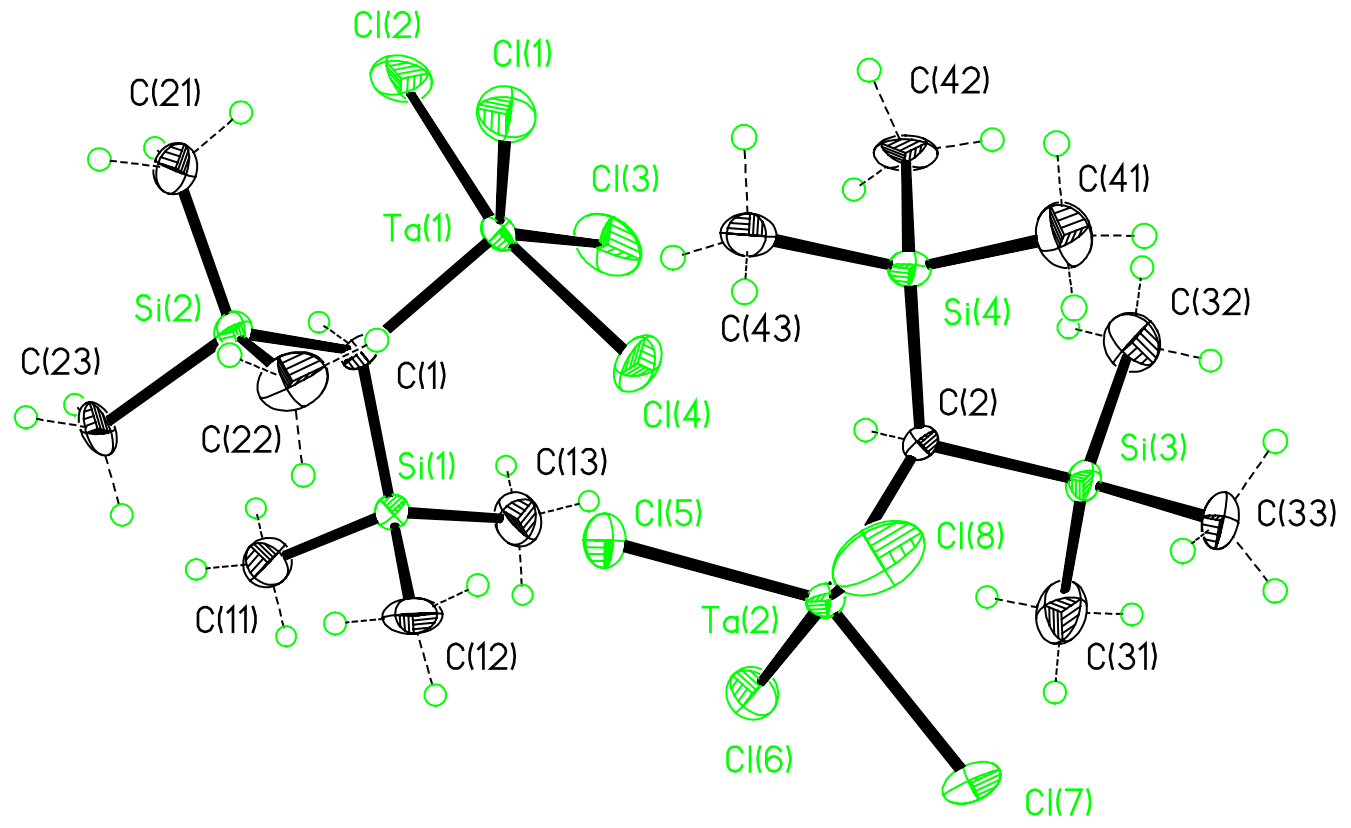

Abbildung 1: Struktur von 1 im Festkörper

Tabelle 2: Ausgewählte Bindungslängen (pm) und -winkel $\left(^{\circ}\right)$ von Verbindung 1 (Mittelwerte)

$\begin{array}{lc}\mathrm{Ta}(1)-\mathrm{C}(1), \mathrm{Ta}(2)-\mathrm{C}(2) & 204.6(2) \\ \mathrm{Ta}(1)-\mathrm{Cl}(1), \mathrm{Ta}(2)-\mathrm{Cl}(7) & 229.9(7) \\ \mathrm{Ta}(1)-\mathrm{Cl}(2), \mathrm{Ta}(2)-\mathrm{Cl}(6) & 231.6(2) \\ \mathrm{Ta}(1)-\mathrm{Cl}(3), \mathrm{Ta}(2)-\mathrm{Cl}(5) & 229.3(7) \\ \mathrm{Ta}(1)-\mathrm{Cl}(4), \mathrm{Ta}(2)-\mathrm{Cl}(8) & 231.1(2) \\ \mathrm{Cl}(2)-\mathrm{Ta}(1)-\mathrm{Cl}(4), \mathrm{Cl}(6)-\mathrm{Ta}(2)-\mathrm{Cl}(8) & 165.01(5) \\ \mathrm{Cl}(1)-\mathrm{Ta}(1)-\mathrm{Cl}(3), \mathrm{Cl}(5)-\mathrm{Ta}(2)-\mathrm{Cl}(7) & 138.20(4) \\ \mathrm{C}(1)-\mathrm{Ta}(1)-\mathrm{Cl}(1), \mathrm{C}(2)-\mathrm{Ta}(2)-\mathrm{Cl}(7) & 111.6(7) \\ \mathrm{C}(1)-\mathrm{Ta}(1)-\mathrm{Cl}(2), \mathrm{C}(2)-\mathrm{Ta}(2)-\mathrm{Cl}(6) & 97.9(7) \\ \mathrm{C}(1)-\mathrm{Ta}(1)-\mathrm{Cl}(3), \mathrm{C}(2)-\mathrm{Ta}(2)-\mathrm{Cl}(5) & 110.1(7) \\ \mathrm{C}(1)-\mathrm{Ta}(1)-\mathrm{Cl}(4), \mathrm{C}(2)-\mathrm{Ta}(2)-\mathrm{Cl}(8) & 97.0(2)\end{array}$


Das Tantal-Metallzentrum liegt im Mittelpunkt einer verzerrten trigonalen Bipyramide. Bei der Diskussion der Bindungslängen- und winkel soll nur ein Molekül betrachtet werden. Erwartungsgemäß findet man den Liganden aufgrund seines größeren Raumbedarfs in der Äquatorialebene. Die Chloratome $\mathrm{Cl}(1)$ und $\mathrm{Cl}(3)$ nehmen äquatoriale und 2 und 4 axiale Positionen ein. Die Tantal-Chlor-Bindungsabstände der äquatorialen Chloratome sind gegenüber den axialen um 2 pm verkürzt. Auffälligerweise ist der Winkel $\mathrm{Cl}(1)-\mathrm{Ta}(1)-\mathrm{Cl}(3)$ um $27^{\circ}$ größer als der Winkel $\mathrm{C}(1)-\mathrm{Ta}(1)-\mathrm{Cl}(1)$, was bedeutet, daß die Chloratome in der Äquatorialebene mehr Raum einnehmen als der Ligand. Der Bis-Ligand bewirkt außerdem eine Verzerrung der trigonalen Bipyramide, die durch eine Verkleinerung des $\mathrm{Cl}(2)-\mathrm{Ta}(1)-\mathrm{Cl}(4)$ Winkels um $15^{\circ}$ auf $165^{\circ}$ zum Ausdruck kommt. Die Torsionswinkel $\mathrm{Si}(2)-\mathrm{C}(1)-\mathrm{Ta}(1)-\mathrm{Cl}(1)$ und $\mathrm{Si}(1)-\mathrm{C}(1)-\mathrm{Ta}(1)-\mathrm{Cl}(3)$ betragen $11.19^{\circ}$ bzw. $48.78^{\circ}$. Somit liegt $\operatorname{Si}(2)$ fast in der Äquatorialebene und $\mathrm{Si}(1)$ recht genau zwischen Äquatorial- und Axialebene.

\subsubsection{Röntgenographische Charakterisierung von $\left(\mathrm{BisTaCl}_{3}\right)_{2}(\mu \mathrm{O}) \mathrm{BisTaCl}_{4}(3)$}

Während eines Kristallisationsversuchs von Verbindung 1 aus $n$-Hexan war innerhalb von einigen Wochen Luftfeuchtigkeit in das Reaktionsgefäß eingedrungen. Die röntgenographische Untersuchung der dabei angefallenen Kristalle zeigte, daß sich ein Cokristallisat gebildet hatte, das aus zwei Molekülen $\mathrm{BisTaCl}_{4}$ und einem Molekül $\left(\mathrm{BisTaCl}_{3}\right)_{2} \mu \mathrm{O}$ in der ermittelten Elementarzelle besteht. Auf eine vollständige spektroskopische und analytische Charakterisierung von $\mathbf{3}$ sowie auf weitere Hydrolyseversuche von 1 wurde verzichtet, weil dies nicht Schwerpunkt dieser Arbeit sein sollte. 


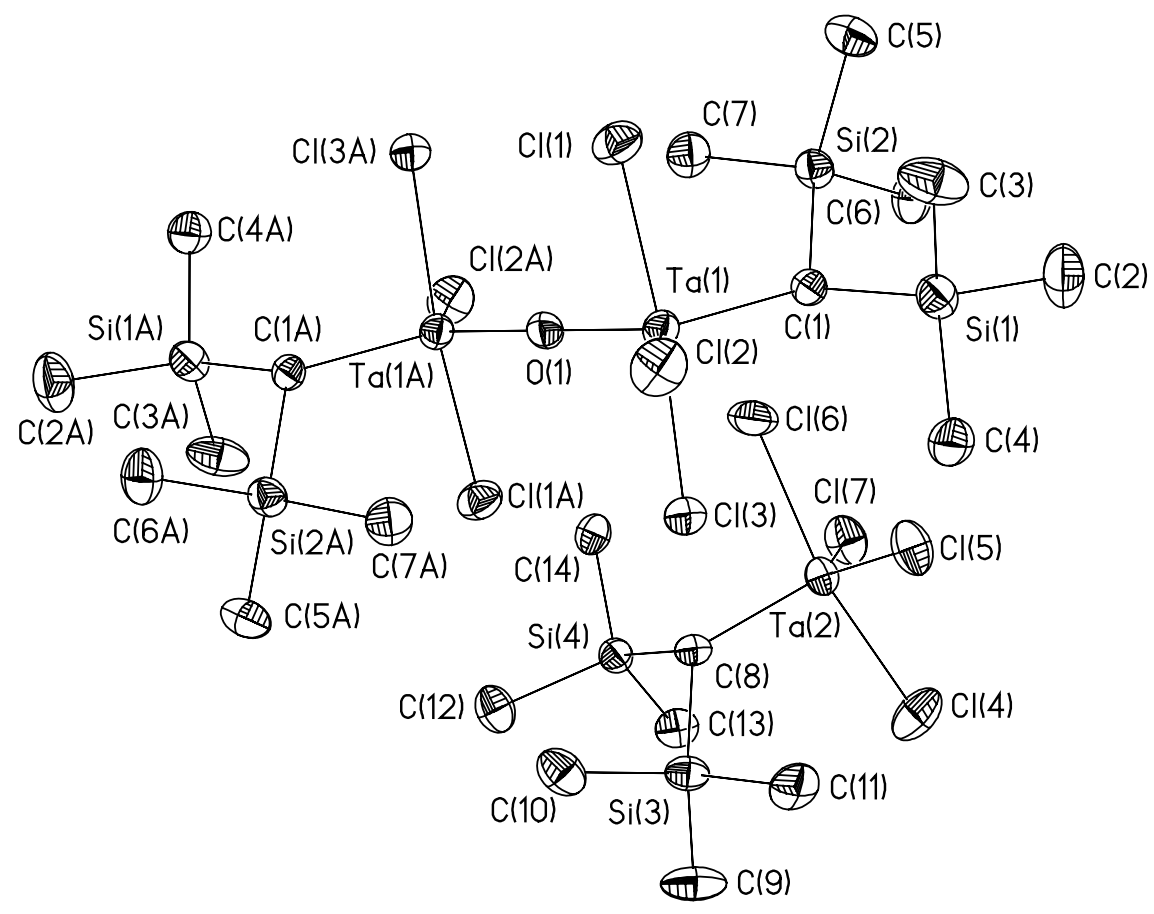

Abbildung 2: Struktur von 3 im Festkörper

Tabelle 3: Bindungsabstände $(\mathrm{pm})$ und -winkel $\left(^{\circ}\right)$ von 3

$\begin{array}{llll}\mathrm{Ta}(1)-\mathrm{O}(1) & 188.13(6) & \mathrm{Ta}(2)-\mathrm{C}(8) & 202.1(5) \\ \mathrm{Ta}(1)-\mathrm{C}(1) & 205.9(5) & \mathrm{Ta}(2)-\mathrm{Cl}(4) & 230.60(17) \\ \mathrm{Ta}(1)-\mathrm{Cl}(1) & 233.53(16) & \mathrm{Ta}(2)-\mathrm{Cl}(5) & 229.38(15) \\ \mathrm{Ta}(1)-\mathrm{Cl}(2) & 231.00(15) & \mathrm{Ta}(2)-\mathrm{Cl}(6) & 233.90(17) \\ \mathrm{Ta}(1)-\mathrm{Cl}(3) & 233.62(16) & \mathrm{Ta}(2)-\mathrm{Cl}(7) & 230.48(15) \\ \mathrm{O}(1)-\mathrm{Ta}(1)-\mathrm{C}(1) & 115.55(13) & \mathrm{O}(1)-\mathrm{Ta}(1)-\mathrm{Cl}(3) & 89.24(4) \\ \mathrm{O}(1)-\mathrm{Ta}(1)-\mathrm{Cl}(2) & 128.17(4) & \mathrm{Cl}(2)-\mathrm{Ta}(1)-\mathrm{Cl}(1) & 86.77(6) \\ \mathrm{C}(1)-\mathrm{Ta}(1)-\mathrm{Cl}(2) & 116.26(14) & \mathrm{Cl}(1)-\mathrm{Ta}(1)-\mathrm{Cl}(3) & 172.97(5) \\ \mathrm{Cl}(2)-\mathrm{Ta}(1)-\mathrm{Cl}(3) & 88.80(6) & \mathrm{C}(8)-\mathrm{Ta}(2)-\mathrm{Cl}(5) & 112.01(14) \\ \mathrm{Cl}(7)-\mathrm{Ta}(2)-\mathrm{Cl}(6) & 87.42(6) & \mathrm{Si}(3)-\mathrm{C}(8)-\mathrm{Ta}(2) & 120.5(2) \\ \mathrm{Cl}(4)-\mathrm{Ta}(2)-\mathrm{Cl}(6) & 168.44(6) & \mathrm{Cl}(7)-\mathrm{Ta}(2)-\mathrm{Cl}(4) & 86.39(6) \\ \mathrm{Cl}(5)-\mathrm{Ta}(2)-\mathrm{Cl}(7) & 134.94(5) & & \end{array}$


Verbindung 3 kristallisiert in der triklinen Raumgruppe $P \overline{1}$. Die Metallzentren sind verzerrt trigonal bipyramidal koordiniert. Die Verzerrung zeigt sich in den Bindungswinkeln $\mathrm{Cl}(4)-\mathrm{Ta}(2)-\mathrm{Cl}(6), 168.44^{\circ}$ und $\mathrm{Cl}(1)-\mathrm{Ta}(1)-\mathrm{Cl}(3), 172.97^{\circ}$. Durch die Einführung des Sauerstoffs weichen die Bindungsabstände der Ta-C und Ta-Cl Bindungen nur geringfügig von denen der sauerstofffreien Spezies ab. Die Torsionswinkel der sauerstoffverbrückten Verbindung betragen für $\mathrm{Si}(2)-\mathrm{C}(1)-\mathrm{Ta}(1)-\mathrm{O}(1) 45.27^{\circ}$ und für $\mathrm{Si}(1)-\mathrm{C}(1)-\mathrm{Ta}(1)-\mathrm{Cl}(2) 5.79^{\circ}$. Die $\mathrm{SiMe}_{3}$-Gruppen nehmen die gleiche Lage zu der trigonal-bipyramidalen Umgebung des Metallzentrums ein wie in Verbindung 1.

\subsubsection{Darstellung von $\mathrm{BisNbCl}_{4}(4)$ und $\mathrm{BisNbBr}_{4}(5)$}

Bis-Liganden tragende Niobkomplexe sind bislang unbekannt. Dieses könnte mit einer geringeren Stabilität der Niob- gegenüber den Tantalalkylen begründet werden. In diesem Zusammenhang erschien es sinnvoll zu überprüfen, in wie weit sich $\mathrm{NbCl}_{5}$ und $\mathrm{NbBr}_{5}$ mit $\mathrm{Bis}_{2} \mathrm{Zn}$ alkylieren lassen. Hierbei gelang die einfache Substitution eines Halogenids gegen einen Bis- Liganden.
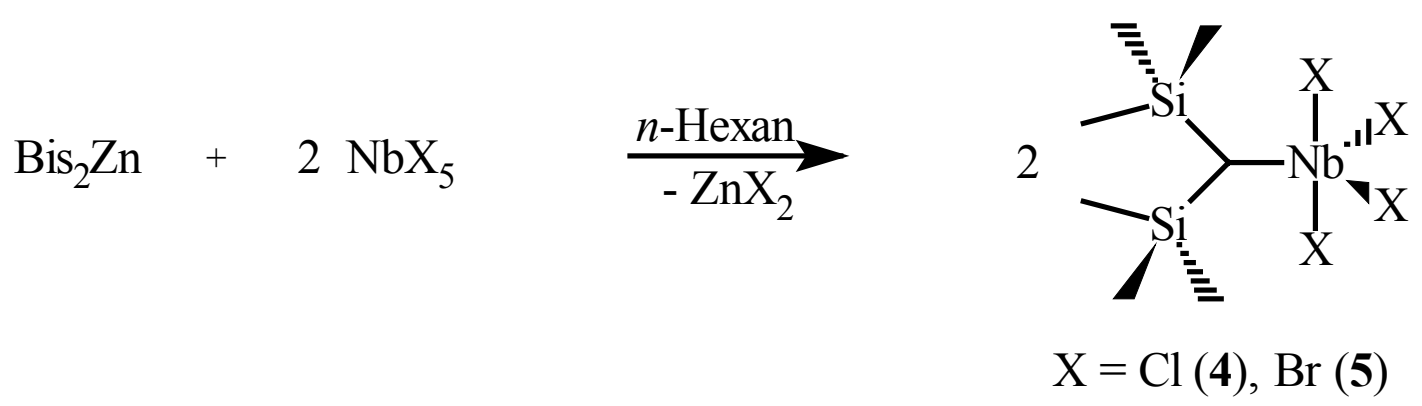

Die Verbindungen 4 und 5 wurden als orangefarbige, luft- und wasserempfindliche Feststoffe isoliert, die bei Raumtemperatur der Zersetzung unterliegen. Beide Verbindungen wurden durch Elementaranalysen, IR- und NMR-Spektroskopie, sowie Massenspektrometrie charakterisiert.

Nach Umkristallisation aus $n$-Hexan bei $-30{ }^{\circ} \mathrm{C}$ wurden für eine Einkristallröntgenstrukturanalyse geeignete Kristalle von $\mathbf{4}$ isoliert. Abbildung 3 zeigt die Struktur von 4 im Kristall. Charakteristische Bindungslängen und -winkel sind in Tabelle 4 aufgeführt. 


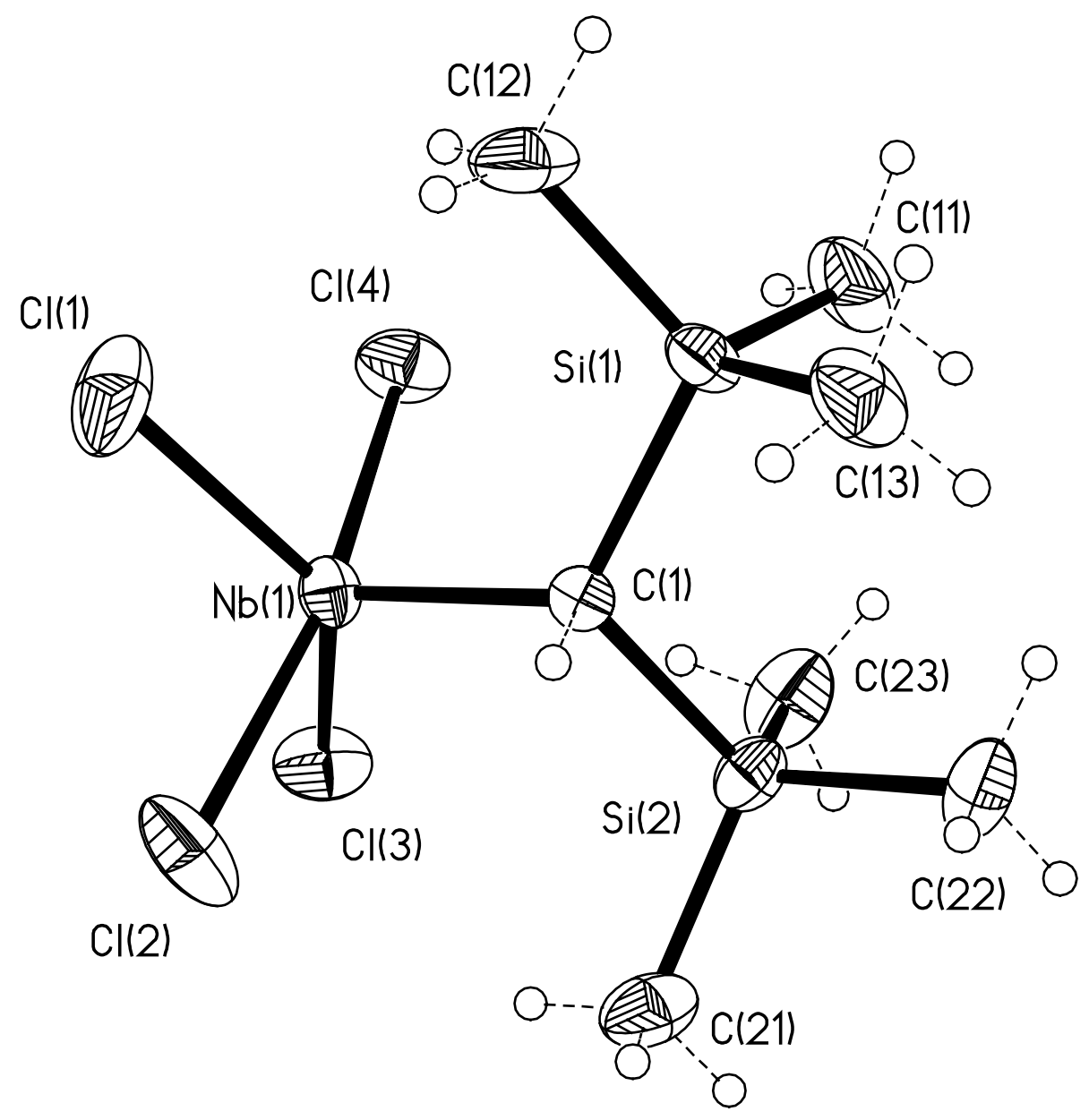

Abbildung 3: Struktur von $\mathrm{BisNbCl}_{4}$ im Festkörper

Tabelle 4: Charakteristische Bindungslängen (pm) und -winkel $\left(^{\circ}\right)$ von $\mathrm{BisNbCl}_{4}$ (4)

$\begin{array}{ll}\mathrm{Nb}(1)-\mathrm{C}(1) & 201.9(4) \\ \mathrm{Nb}(1)-\mathrm{Cl}(2) & 232.65(13) \\ \mathrm{Nb}(1)-\mathrm{Cl}(4) & 233.04(13) \\ \mathrm{Cl}(2)-\mathrm{Nb}(1)-\mathrm{Cl}(4) & 165.95(5) \\ \mathrm{C}(1)-\mathrm{Nb}(1)-\mathrm{Cl}(1) & 112.70(10) \\ \mathrm{C}(1)-\mathrm{Nb}(1)-\mathrm{Cl}(2) & 97.58(10)\end{array}$

$\mathrm{Nb}(1)-\mathrm{Cl}(1)$

$\mathrm{Nb}(1)-\mathrm{Cl}(3)$

229.10(13)

233.54(12)

$\mathrm{Cl}(1)-\mathrm{Nb}(1)-\mathrm{Cl}(3) \quad 133.66(5)$

$\mathrm{C}(1)-\mathrm{Nb}(1)-\mathrm{Cl}(3) \quad 113.64(10)$

$\mathrm{C}(1)-\mathrm{Nb}(1)-\mathrm{Cl}(2) \quad 97.58(10)$ 
Verbindung 4 kristallisiert monomer in der monoklinen Raumgruppe $P 2_{1} / c$. Aus dem großen Platzbedarf des Bis-Liganden resultiert eine verzerrt trigonal-bipyramidale Struktur um das Metallatom. Bei einem Vergleich der Bindungslängen fällt auf, daß sich die $\mathrm{Nb}(1)-\mathrm{Cl}(3)$ - und $\mathrm{Nb}(1)-\mathrm{Cl}(1)$-Bindungsabstände um $4 \mathrm{pm}$ unterscheiden, obwohl beide Chloratome äquatoriale Positionen einnehmen. Vielmehr ist die $\mathrm{Nb}(1)-\mathrm{Cl}(3)$-Bindungslänge mit der zu den äquatorialen Chloratomen $\mathrm{Cl}(2)$ und $\mathrm{Cl}(4)$ vergleichbar. Die Bindungwinkel der Verbindungen 1 und $\mathbf{4}$ stimmen gut überein.

\subsubsection{Darstellung von $\mathrm{BisTiCl}_{3}(6)$}

Durch Umsetzen von $\mathrm{Bis}_{2} \mathrm{Zn}$ mit $\mathrm{TiCl}_{4}$ in $n$-Hexan im molaren Verhältnis $1: 2.5$ wurde das Monoalkylierungsprodukt $\mathrm{BisTiCl}_{3}$ in guter Ausbeute hergestellt. Es wurde ein Überschuß an $\mathrm{TiCl}_{4}$ eingesetzt, um eine höhere Alkylierung zu verhindern und um das Zinkorganyl vollständig zu verbrauchen.

$$
\mathrm{Bis}_{2} \mathrm{Zn}+2 \mathrm{TiCl}_{4}
$$

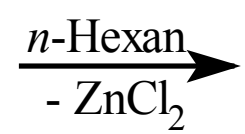

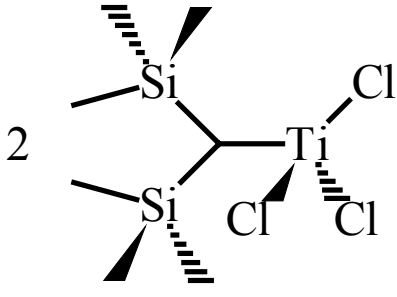

6

Bei einer Reaktionsführung mit einem molaren Verhältnis 1:1 entsteht kein disubstituiertes Produkt. Ein Teil des Zinkorganyls kann zurückgewonnen werden.

Der Peak im Massenspektrum bei m/z 299 läßt sich dem Fragment $\left(\mathrm{M}^{+}-\mathrm{Me}\right)$ zuordnen und bestätigt damit, daß eine Monosubstitution stattgefunden hat. Die ${ }^{1} \mathrm{H}-$ und ${ }^{29} \mathrm{Si}$ NMR-Spektren zeigen die erwarteten Signale im korrekten Intensitätsverhältnis. Die Elementaranalyse liefert zu hohe Werte für Kohlenstoff und Wasserstoff und zu niedrige für Chlor. Trotz zweimaliger Destillation konnten keine schlüssigen Werte erhalten werden.

Aufgrund des geringen Temperaturunterschiedes zwischen Schmelzpunkt und Kristallisationstemperatur bildeten sich keine für eine Einkristallröntgenstrukturanalyse geeigneten Kristalle. 


\subsubsection{Weitere Alkylierungsversuche mit $\mathrm{Bis}_{2} \mathrm{Zn}$}

Es ist allgemein schwierig, Reaktionen mit reinen Metallfluoriden durchzuführen, weil sie eine polymere Struktur aufweisen. Im Gegensatz dazu bildet Tantalpentafluorid ein tetrameres Aggregat [56], weshalb hier ein Alkylierungsversuch vielversprechend erschien. Die Reaktion wurde in $n$-Hexan zunächst bei Raumtemperatur und später in der Siedehitze durchgeführt. Die Ausgangsverbindungen blieben unverändert zurück.

Außerdem wurde versucht, $\mathrm{ZrCl}_{4}, \mathrm{HfCl}_{4}, \mathrm{WCl}_{6}, \mathrm{ReCl}_{5}, \mathrm{SmCl}_{3}, \mathrm{LaCl}_{3}$ and $\mathrm{SiCl}_{4}$ mit $\mathrm{Bis}_{2} \mathrm{Zn}$ in $n$-Hexan zu alkylieren. Selbst nach mehrstündigem Erhitzen unter Rückfluß konnte keine Reaktion beobachtet werden und die Edukte wurden unverändert zurückgewonnen. 


\subsection{Metathetische Fluorierungen mit $\mathrm{Me}_{3} \mathrm{SnF}$ (7)}

Im Rahmen der Katalysatorentwicklung für die Olefinpolymerisation wurden viele Fluorokomplexe der 4. Gruppe dargestellt. Deutlich weniger Arbeiten beschäftigen sich mit entsprechenden Komplexen der 5. Gruppe. Die Chlorid-Fluorid-Metathese wurde zuerst für die Darstellung der $\pi$-Komplexe $\mathrm{Cp}^{*} \mathrm{NbF}_{4}$ und $\mathrm{Cp}^{*} \mathrm{TaF}_{4}[22,23]$ mit Hilfe von $\mathrm{AsF}_{3}$ realisiert. Später wurde die Synthese durch Verwendung von $\mathrm{Me}_{3} \mathrm{SnF}$ als Fluorierungsmittel für die Fluorokomplexe $\mathrm{Cp}^{*} \mathrm{VF}_{2}[57]$ und $\mathrm{Cp}^{*} \mathrm{TaF}_{4}[58]$ verbessert.

Als erster Vertreter eines vergleichbaren $\sigma$-Komplexes wurde $\left(\mathrm{Me}_{3} \mathrm{CCH}_{2}\right)_{3} \mathrm{TaF}_{2}$ von Schrock et al. in einem Versuch zur Herstellung von $\left[\left(\mathrm{Me}_{3} \mathrm{CCH}_{2}\right)_{4} \mathrm{Ta}\right]^{+}\left[\mathrm{BF}_{4}\right]^{-}$aus dem Carbenkomplex $\left(\mathrm{Me}_{3} \mathrm{CCH}_{2}\right)_{3} \mathrm{Ta}=\mathrm{CHCMe}_{3}$ und $\mathrm{HBF}_{4}$ in geringer Ausbeute dargestellt [59]. Im Arbeitskreis Roesky wurden $\mathrm{Ph}_{3} \mathrm{TaF}_{2}, p-\mathrm{Tol}_{3} \mathrm{TaF}_{2}$ und $\left(\mathrm{Me}_{3} \mathrm{SiCH}_{2}\right)_{3} \mathrm{TaF}_{2}$ aus den entsprechenden Chloriden in Metathesereaktionen mit $\mathrm{Me}_{3} \mathrm{SnF}$ synthetisiert [60].

Wie anfangs erwähnt, ist $\mathrm{Me}_{3} \mathrm{SnF}$ in der Lage, Methylgruppen zu substituieren. Das erste Beispiel für eine Fluorierung mit 7 war die Methylsubstitution in $\mathrm{GaMe}_{3}$ unter Erhalt von $\mathrm{Me}_{2} \mathrm{GaF}$ [61]. In diesem Zusammenhang erschien es interessant zu überprüfen, inwieweit andere Alkyl- oder Arylgruppen ersetzt werden. Bei der Herstellung von $\mathrm{Ph}_{3} \mathrm{TaF}_{2}$ und $p-\mathrm{Tol}_{3} \mathrm{TaCl}_{3}$ konnte keine Substitution des Arylrests beobachtet werden. Im Gegensatz dazu konnte $\mathrm{Ns}_{3} \mathrm{TaF}_{2}$ nie rein dargestellt werden, da als Beiprodukt überraschenderweise $\mathrm{Ns}_{3} \mathrm{SnCl}$ (8) kristallographisch nachgewiesen wurde, das sich destillativ nicht abtrennen ließ [60]. Dieser Befund zeigt, daß am Zinn-Metallzentrum nicht nur Fluorid gegen Chlorid, sondern auch Methyl gegen Neosilyl ausgetauscht wurde. Die auf diesem Weg isolierte Menge an $\mathrm{Ns}_{3} \mathrm{SnCl}$ war im Vergleich zum Hauptprodukt gering, so daß sich diese Reaktionsführung sicherlich nicht zur Herstellung von 8 eignet. $\mathrm{Ns}_{3} \mathrm{SnCl}$ wurde bereits im Jahre 1971 aus $\mathrm{Ns}_{4} \mathrm{Sn}$ und $\mathrm{SnCl}_{4}$ in guter Ausbeute dargestellt [62]. Eine Einkristallröntgenstrukturanalyse von 8 wurde bislang noch nicht in der Literatur beschrieben.

$\begin{array}{lll}\text { Hauptreaktion: } & \mathrm{Ns}_{3} \mathrm{TaCl}_{2}+2 \mathrm{Me}_{3} \mathrm{SnF} \longrightarrow \mathrm{Ns}_{3} \mathrm{TaF}_{2}+2 \mathrm{Me}_{3} \mathrm{SnCl} \\ \text { Nebenreaktion: } & \mathrm{Ns}_{3} \mathrm{TaCl}_{2}+2 \mathrm{Me}_{3} \mathrm{SnF} \longrightarrow \mathrm{Ns}_{3} \mathrm{SnCl}+\quad ?\end{array}$




\subsubsection{Diskussion der Einkristallröntgenstrukturanalyse von $\mathrm{Ns}_{3} \mathrm{SnCl}(8)$}

Aus einer Reaktionsmischung, die sowohl $\mathrm{Ns}_{3} \mathrm{TaF}_{2}$ als auch $\mathrm{Ns}_{3} \mathrm{SnCl}$ enthielt, konnten bei $-30{ }^{\circ} \mathrm{C}$ aus $n$-Hexan nach einen Zeitraum von drei Monaten farblose, nadelförmige Kristalle gewonnen werden, die für eine Einkristallröntgenstrukturanalyse geeignet waren. Verbindung 8 kristallisiert in der hexagonalen Raumgruppe $P 6_{3} / m$. Die Zinnmetallzentren sind trigonalbipyramidal koordiniert und über Ecken zu polymeren Ketten verknüpft. Dabei liegen die Neosilylgruppen aufgrund ihres größeren Platzbedarfs in der Äquatorialebene und die Chloratome nehmen axiale Positionen ein. Erstaunlich ist die Ausbildung einer idealen trigonalen Bipyramide mit C-Sn-C Winkeln von $120.0^{\circ}$ und Cl-Sn-C Winkeln von 90.0 $0^{\circ}$ Die Chloratome wirken verbrückend und sind exakt mittig zwischen den Metallzentren positioniert. In Verbindung 8 betragen sämtliche Sn-Cl Bindungsabstände 275.31(4) pm. Die Sn-Cl-Sn und Cl-Sn-Cl Bindungswinkel von $180^{\circ}$ zeigen, daß die trigonal bipyramidalen Einheiten exakt linear verbunden sind. Im Gegensatz dazu liegen $\mathrm{Bis}_{3} \mathrm{SnCl}$ [63] und $\mathrm{Ph}_{3} \mathrm{SnCl}$ [64] im Festkörper monomer vor und sind tetraedrisch koordiniert. $\mathrm{Me}_{3} \mathrm{SnCl}$ bildet ebenfalls ein Polymer aus, allerdings findet man unterschiedliche Sn-Cl-Bindungsabstände von 243 und 326 pm und die Ausbildung einer Zickzackkette [65].

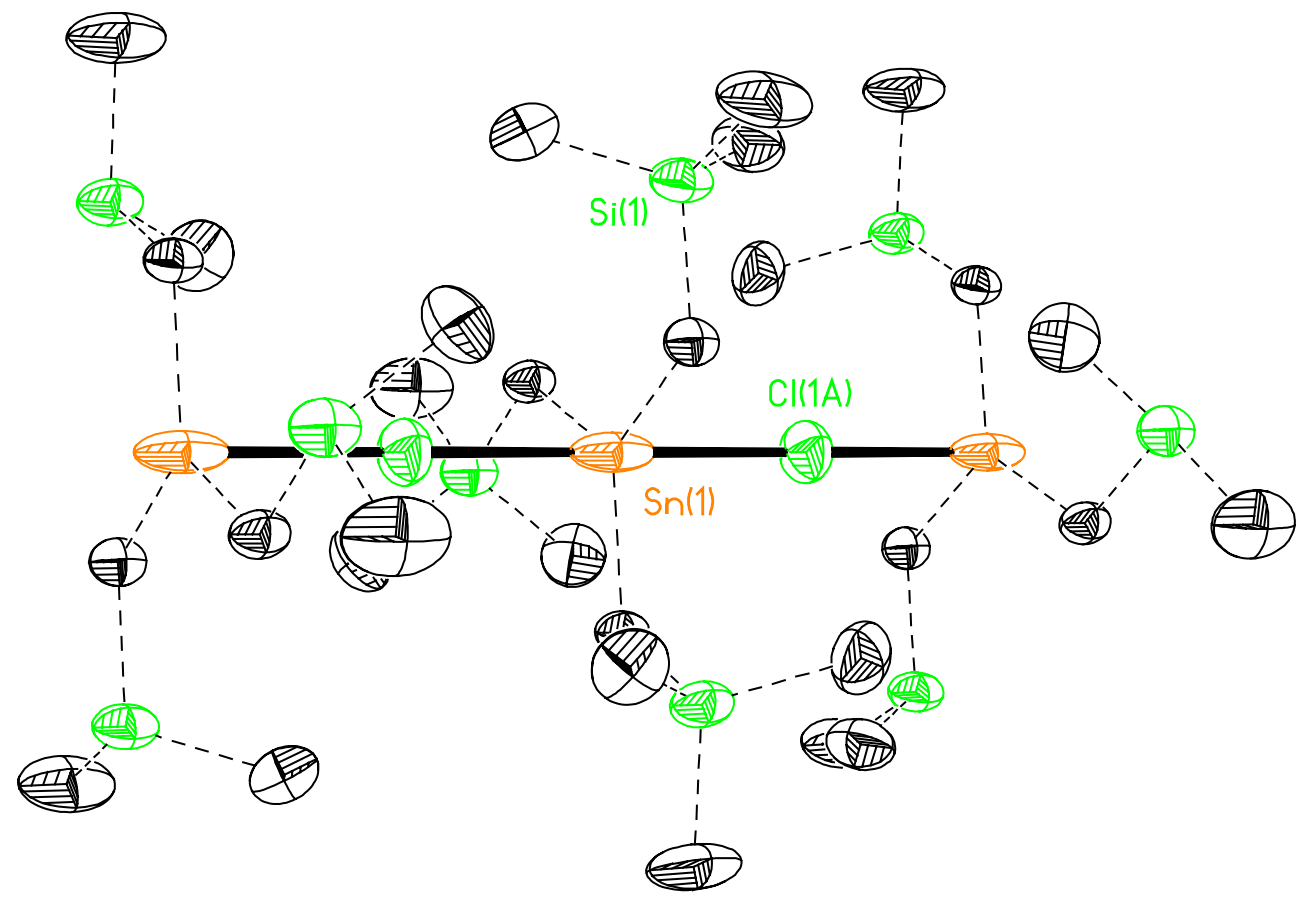

Abbildung 4: Ausschnitt der polymeren Struktur von 8 im Festkörper 
Tabelle 5: Charakteristische Bindungslängen (pm) und -winkel $\left({ }^{\circ}\right)$ von $\mathrm{Ns}_{3} \mathrm{SnCl}(\mathbf{8})$

$\begin{array}{lllr}\operatorname{Sn}(1)-C(1) & 211.8(6) & \mathrm{C}(1)-\operatorname{Sn}(1)-\mathrm{C}(1 \#) & 120.0 \\ \operatorname{Sn}(1)-\mathrm{Cl}(1) & 275.31(4) & \mathrm{C}(1)-\operatorname{Sn}(1)-\mathrm{Cl}(1) & 90.0 \\ \operatorname{Sn}(1 \#)-\mathrm{Cl}(1) & 275.31(4) & \mathrm{Sn}(1)-\mathrm{Cl}(1)-\operatorname{Sn}(1 \#) & 180.0\end{array}$

\subsubsection{Darstellung von $\mathrm{BisTaF}_{4}(9)$}

Die Reaktionen von Alkyltantalchloriden mit Trimethylzinnfluorid werden von der Anzahl und dem sterischen Anspruch der organischen Liganden beeinflußt. Die Synthese der Trialkyltantaldifluoride der allgemeinen Formel $\left(\mathrm{RCH}_{2}\right)_{3} \mathrm{TaF}_{2}\left(\mathrm{R}=\mathrm{Ph}, p\right.$-Tol, $\left.\mathrm{Me}_{3} \mathrm{Si}\right)$ gelingt problemlos. Entsprechende zwei oder eine organische Gruppe tragende Tantalfluoride lassen sich auf diesem Weg nicht darstellen. Bei der Reaktion von $\mathrm{NsTaCl}_{4}$ mit 7 wird eine Substitution der Neosilylgruppe beobachtet [60]. Im Gegensatz dazu wird bei der Fluorierung von $\mathrm{BisTaCl}_{4}$ mit 7 die organische Gruppe nicht ausgetauscht und man erhält $\mathrm{BisTaF}_{4}(9)$. Das mag sowohl an dem größeren sterischen Anspruch von Bis gegenüber Ns liegen als auch an einer Stabilisierung der Metall-Kohlenstoff Bindung (Kap. 2.1.).

Verbindung 9 wurde spektroskopisch und röntgenstrukturell charakterisiert. Das lösungsmittelfreie Produkt ergibt eine korrekte Elementaranalyse. Im ${ }^{1} \mathrm{H}-\mathrm{NMR}$-Spektrum findet man zwei Singuletts bei $\delta 0.28$ und $3.06 \mathrm{ppm}$, die den Protonen des Bis-Liganden zugeordnet werden können. Das ${ }^{19}$ F-NMR-Spektrum zeigt ein breites Singulett bei $\delta 81.19$ ppm. Im Gegensatz zu Ns $\mathrm{TaF}_{2}$ kann hier keine H-F-Kopplung beobachtet werden [60].

Von Verbindung 9 konnte eine Einkristallröntgenstrukturanalyse angefertigt werden. Dafür geeignete Kristalle erhielt man aus $n$-Hexan bei $-30{ }^{\circ} \mathrm{C}$. $\mathrm{BisTaF}_{4}$ kristallisiert in der Raumgruppe $P 2_{1} / c$. Alle Tantal-Metallzentren befinden sich in einer oktaedrischen Umgebung. Die einzelnen Moleküle sind über Fluoridbrücken verknüpft. Die TaF ${ }^{\cdots}$ TaBindungslänge in 9 ist mit 242 pm erstaunlich lang und sogar um 20 pm länger als in $\mathrm{Cp}^{*} \mathrm{TaF}_{4} \times 2 \mathrm{AsF}_{3}$ [22]. Alle Bindungslängen zu endständigen Fluoratomen besitzen mit 187 pm einen ähnlichen Wert wie in vergleichbaren Verbindungen [22]. Der $\mathrm{Cl}(1)-\mathrm{Ta}(1)-\mathrm{F}(5)$ Bindungswinkel von $171^{\circ}$ deutet auf eine leichte Verzerrung des Oktaeders hin. Der $\mathrm{Ta}(1)-\mathrm{F}(5)-\mathrm{Ta}(2)$ Bindungswinkel von $141^{\circ}$ zeigt an, daß die Oktaeder zu einer Zickzackkette verknüpft sind. Die anorganischen Zentren sind von lipophilen Bis-Liganden umgeben, 
woraus man auf eine gute Löslichkeit in organischen Lösungsmitteln schließen kann. In Abbildung 5 ist die Festkörperstruktur von $9 \mathrm{zu}$ sehen und wichtige Bindungslängen und Winkel sind in Tabelle 6 aufgeführt.

Tabelle 6: Ausgewählte Bindungslängen (pm) und -winkel $\left({ }^{\circ}\right)$ von 9

$\begin{array}{lllr}\mathrm{Ta}(1)-\mathrm{F}(1) & 197.1(11) & \mathrm{Ta}(2)-\mathrm{F}(5)-\mathrm{Ta}(1) & 141.0(5) \\ \mathrm{Ta}(1)-\mathrm{F}(2) & 186.0(11) & \mathrm{F}(2)-\mathrm{Ta}(1)-\mathrm{F}(3) & 91.1(5) \\ \mathrm{Ta}(1)-\mathrm{F}(3) & 186.4(11) & \mathrm{F}(2)-\mathrm{Ta}(1)-\mathrm{F}(4) & 151.1(5) \\ \mathrm{Ta}(1)-\mathrm{F}(4) & 186.7(11) & \mathrm{C}(1)-\mathrm{Ta}(1)-\mathrm{F}(5) & 171.4(6) \\ \mathrm{Ta}(1)-\mathrm{F}(5) & 241.8(11) & \mathrm{F}(3)-\mathrm{Ta}(1)-\mathrm{F}(1) & 172.2(5) \\ \mathrm{Ta}(1)-\mathrm{C}(1) & 205 .(2) & & \end{array}$

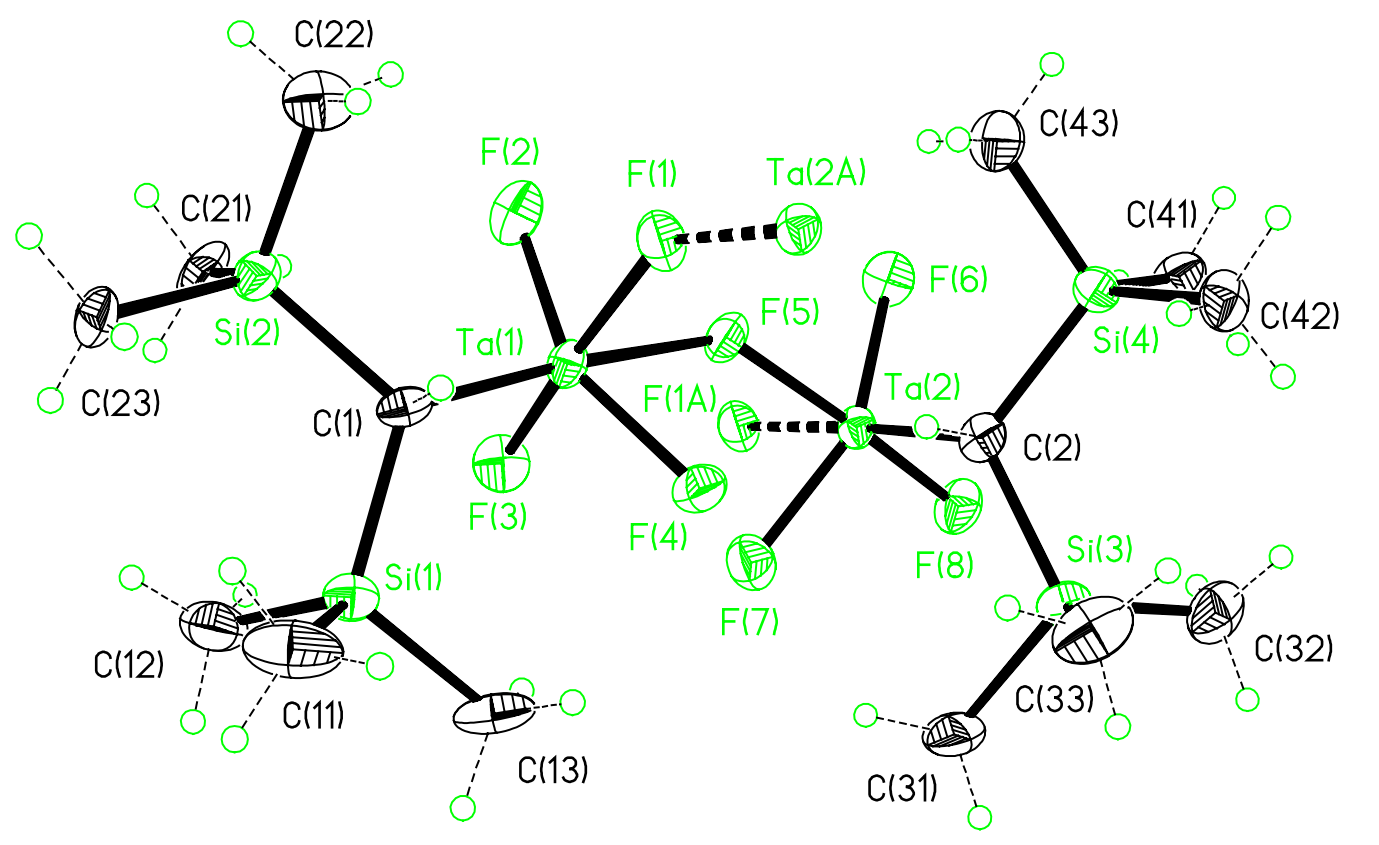

Abbildung 5: Festkörperstruktur von $\mathrm{BisTaF}_{4}(9)$

Da die Struktur von 9 Fluoratome in einer chemisch unterschiedlichen Umgebung zeigt, sollte überprüft werden, inwieweit diese durch eine ${ }^{19}$ F-NMR-Tieftemperaturmessung 
genauer charakterisiert werden können. Das Signal bei $\delta 81.19 \mathrm{ppm}$ wird bei $-95{ }^{\circ} \mathrm{C}$ in $\mathrm{CDCl}_{3}$ in drei Signale bei $\delta$-29, 103 und 123 ppm aufgespalten, die in einem Intensitätsverhältnis von $1: 2: 1$ vorliegen. Die Koaleszenztemperatur beträgt $65^{\circ} \mathrm{C}$. Das stark hochfeldverschobene Signal bei $\delta-29$ ppm stammt von dem verbrückend wirkenden Fluoratom F(1). Die Fluoratome $F(2)$ und $F(4)$, die jeweils einem endständigen und zwei verbrückenden Fluoratomen benachbart sind, ergeben das Signal bei $\delta 103$ ppm. $\mathrm{F}(3)$ unterscheidet sich von $F(2)$ und $F(4)$ dadurch, daß es zwei endständigen und einem verbrückenden Fluoratom benachbart ist. Damit wird das Auftreten eines weiteren Signals bei $\delta 123 \mathrm{ppm}$ für $\mathrm{F}(3)$ plausibel. Bei einer Temperatur von $-95^{\circ} \mathrm{C}$ läßt sich eine Kopplung zwischen $\mathrm{F}(1)$ und $\mathrm{F}(3)$ beobachten $\left({ }^{2} J_{\mathrm{F}-\mathrm{F}}=185 \mathrm{~Hz}\right)$.

\subsubsection{Darstellung von $\left[\left(\mathrm{BisTaF}_{4}\right)_{2} \mathrm{CsF}\right](10)$}

Die Chemie der organometallischen Fluoride weist Komplexe des Wirt-Gast-Typs auf [66]. Bringt man beispielsweise $\mathrm{Cp}^{*} \mathrm{TiF}_{3}$ mit $\mathrm{NaF}$ zur Reaktion, so erhält man $[\mathrm{Na}]^{+}\left[\left(\mathrm{Cp}^{*} \mathrm{TiF}_{3}\right)_{4} \mathrm{NaF}_{2}\right]^{-}[29]$, einen Komplex mit einem internen und einem externen Natriumion. Ein Austausch des externen Natriumions durch $\mathrm{PPh}_{4}{ }^{+}$erfolgt leicht und die Struktur des Austauschproduktes wurde aufgeklärt. Um Fluoride mit einer hohen Gitterenergie wie beispielsweise $\mathrm{CaF}_{2}$ einzusetzen, stellt man sie in situ her. Dieses geschieht durch Fluorierung von $\mathrm{CaCl}_{2}$ mit $\mathrm{Me}_{3} \mathrm{SnF}$ und in Gegenwart von EtCp*TiF 3 erhält man den Komplex [(EtCp* $\left.\left.\mathrm{TiF}_{3}\right)_{4} \mathrm{CaF}_{2}\right]$, in dem das Calcium von 8 Fluoratomen umgeben ist [67]. Es stellte sich nun die Frage, ob ähnliche Komplexe mit Tantal isolierbar sind. Zu diesem Zweck wurde $\mathrm{BisTaF}_{4}$ mit CsF zur Reaktion gebracht.

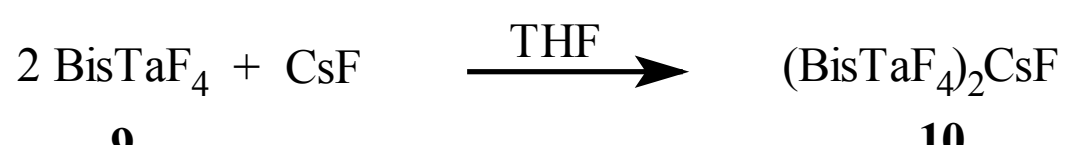
9

10

Im ${ }^{19}$ F-NMR-Spektrum von 10 findet man ein Singulett bei $\delta 45.71 \mathrm{ppm}$, das gegenüber dem Signal von 9 um 35 ppm hochfeldverschoben ist. Ein ${ }^{133}$ Cs-NMR-Spektrum zeigt ein Singulett bei $\delta$-55.6 ppm gegen CsF als Standard. Die NMR-Spektren zeigen, daß 
eine neue Verbindung entstanden ist, die sowohl $\mathrm{CsF}$ als auch $\mathrm{BisTaF}_{4}$ enthält. Eine genauere Aussage über die Zusammensetzung läßt sich anhand des Massenspektrums machen. Dort findet man in geringer Intensität den Molkülpeak für $\left[\left(\mathrm{BisTaF}_{4}\right)_{2} \mathrm{CsF}\right]$ mit einer korrekten Isotopenverteilung, was für die Stabilität dieses Clusters spricht. Die Aufklärung der Struktur im Festkörper scheiterte, weil keine dafür geeigneten Kristalle aus verschiedenen Toluol/THFMischungen erhalten werden konnten.

\subsubsection{Darstellung von $\mathrm{BisNbF}_{4}$ (11)}

Von Niobfluoriden, mit Niob-Kohlenstoff- $\sigma$-Bindung sind bislang nur Komplexe bekannt, die durch Cp-Liganden elektronisch stabilisiert werden. Ein Beispiel ist die Niobverbindung $\mathrm{Cp}_{2} \mathrm{Nb}(\mathrm{F})\left(\mathrm{CF}_{3} \mathrm{C}=\mathrm{C}(\mathrm{H}) \mathrm{CF}_{3}\right)$, die in geringer Ausbeute aus der Reaktion von $\mathrm{Cp}_{2} \mathrm{NbH}_{3}$ mit Hexafluorobutin resultierte [68].

$\mathrm{Ns}_{3} \mathrm{NbF}_{2}$ läßt sich im Vergleich zu Ns $\mathrm{TaF}_{2}$ nicht über eine Fluorierung von $\mathrm{Ns}_{3} \mathrm{NbCl}_{2}$ mit $\mathrm{Me}_{3} \mathrm{SnF}$ darstellen. Bei dieser Reaktion wurde ein schwarzes Öl isoliert, dessen Charakterisierung fehlschlug. Setzt man $\mathrm{BisNbCl}_{4}$ mit vier Äquivalenten 7 bei $-70{ }^{\circ} \mathrm{C}$ in $\mathrm{CH}_{2} \mathrm{Cl}_{2}$ um, so erhält man nach Vakuumsublimation $\mathrm{BisNbF}_{4}(\mathbf{1 1})$ in hochreiner Form.

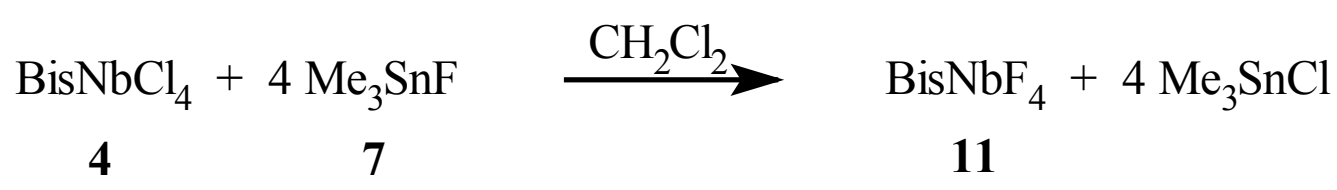

Verbindung 11 ist ein gegen Wasser und Sauerstoff empfindlicher gelber Feststoff. Die Temperaturbeständigkeit ist gegenüber dem entsprechenden Chlorid deutlich höher, so daß 11 eine eintägige Lagerung bei Raumtemperatur ohne äußere Veränderung übersteht. Im Massenspektrum findet man den Peak des Fragments $\left(\mathrm{M}^{+}-\mathrm{Me}\right)$ in der korrekten Isotopenverteilung. Bei der Elementaranalyse stimmen berechnete und ermittelte Werte gut überein. Die ${ }^{1} \mathrm{H}$ - und ${ }^{19}$ F-NMR-Spektren bestätigen die Bildung von 11. Die Aufklärung der Struktur im Festkörper mißlang, weil keine für eine Einkristallstrukturanalyse geeigneten Kristalle gewonnen werden konnten, obwohl verschiedene Kristallisationsversuche aus $n$-Hexan, Toluol und den Lösugsmittelgemischen $n$-Hexan/Toluol $=3 / 1$ und $n-H e x a n / E t_{2} \mathrm{O}$ $=10 / 1$ bei 0 und $-30{ }^{\circ} \mathrm{C}$ durchgeführt wurden. 


\subsubsection{Reaktion von $\mathrm{BisTiCl}_{3}$ (6) mit $\mathrm{Me}_{3} \mathrm{SnF}(7)$}

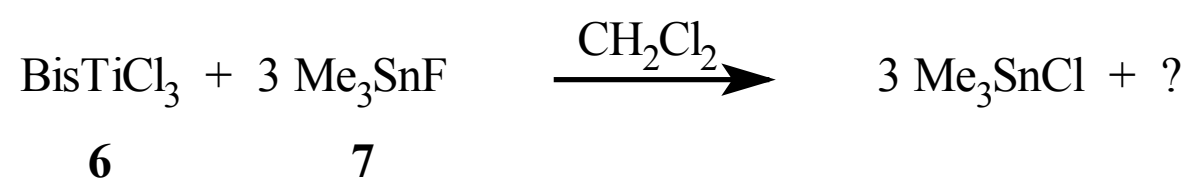

Titanfluoride mit einer $\sigma$-gebundenen Alkylgruppe sind selten. Bislang wurden die Komplexe $\mathrm{Cp}_{2} \mathrm{Ti}(\mathrm{Me}) \mathrm{F}$ und $\mathrm{Cp}_{2} \mathrm{Ti}\left(\mathrm{C}_{6} \mathrm{~F}_{5}\right) \mathrm{F}$ beschrieben [69]. $\mathrm{BisTiCl}_{3}$ wurde mit drei Äquivalenten $\mathrm{Me}_{3} \mathrm{SnF}$ zur Reaktion gebracht. Während die Reaktionsmischung langsam von $0{ }^{\circ} \mathrm{C}$ auf Raumtemperatur gebracht wurde, reagierte das $\mathrm{Me}_{3} \mathrm{SnF}$ ab, was man am Auflösen des Bodensatzes beobachten konnte. Es entstand eine homogene, orangefarbene Lösung. Nachdem alle flüchtigen Bestandteile im Vakuum entfernt worden waren, um das bei der Reaktion entstandene Beiprodukt $\mathrm{Me}_{3} \mathrm{SnCl}$ abzutrennen, erwies sich der Rückstand als in allen gängigen Lösungsmitteln unlöslich. Ein Sublimationsversuch mußte ebenfalls erfolglos abgebrochen werden, nachdem sich vermutlich bei der Reaktion $\mathrm{TiF}_{4}$ gebildet hatte. Auf eine weitere Charakterisierung wurde daher verzichtet.

\subsubsection{Versuch der Fluorierung von $\mathrm{Silox}_{3} \mathrm{TaCl}_{2}\left(\mathrm{Silox}=(t-\mathrm{BuO})_{3} \mathrm{SiO}-\right)$ mit $\mathrm{Me}_{3} \mathrm{SnF}(7)$}

Im Arbeitskreis Wolczanski wurden siloxligandentragende Tantalhydridkomplexe hergestellt und deren Verhalten in Fischer-Tropsch-verwandten Reaktionen untersucht $[70,71]$. Im folgenden soll überprüft werden, ob es möglich ist, in dem Tantal-ChloridSilanolat-System Chlorid gegen Fluorid mit Hilfe von $\mathrm{Me}_{3} \mathrm{SnF}$ auszutauschen. An der Verbindung Silox $\mathrm{TaCl}_{3}$ wurden Fluorierungsversuche mit $\mathrm{AsF}_{3}$ und $\mathrm{NaF}$ unternommen [72]. Dabei konnte $\left(\mathrm{AsCl}_{2}\right)_{2}\left(\mathrm{Silox}_{2} \mathrm{TaF}_{5}\right)$ isoliert werden. Die Reaktion mit NaF ergab ein Produktgemisch, das $\mathrm{Na}_{2} \mathrm{Silox}_{2} \mathrm{TaF}_{5}$ enthielt. Silox $\mathrm{TaCl}_{2}$ wurde mit zwei Äquivalenten $\mathrm{Me}_{3} \mathrm{SnF}$ in Toluol zur Reaktion gebracht. Nachdem bei Raumtemperatur keine Reaktion beobachtet werden konnte, wurde $2 \mathrm{~h}$ unter Rückfluß erhitzt. Da die Umsetzung immer noch unvollständig war, wurde ein weiteres Äquivalent $\mathrm{Me}_{3} \mathrm{SnF}$ zugesetzt und nochmals $6 \mathrm{~h}$ refluxiert. Nach Filtration und Entfernen des Lösungsmittels wurde ein weißer Feststoff isoliert, der mit $279^{\circ} \mathrm{C}$ einen ähnlichen Schmelzpunkt wie das Chlorid $\left(270{ }^{\circ} \mathrm{C}\right)$ aufweist. Allerdings konnte das Beiprodukt $\mathrm{Me}_{3} \mathrm{SnCl}$ anhand seines charakteristischen Geruchs im 
abkondensierten Lösungsmittel nachgewiesen werden. Im ${ }^{1} \mathrm{H}-\mathrm{NMR}-$ Spektrum findet man zwei Singuletts bei $\delta 1.08$ und 1.23 ppm, die vermutlich von unterschiedlichen, Siloxgruppen tragenden Verbindungen stammen. Das Vehältnis der Integrale beträgt $1: 4$. Das ${ }^{19}$ F-NMRSpektrum weist ein Singulett bei $\delta 51.06$ ppm auf, dessen Verschiebung für die Ausbildung einer Ta- $\mathrm{F}_{(\text {endst.) }}$ Bindung spricht. Im Massenspektrum hingegen findet man nur Peaks des Edukts $\left\{\mathrm{m} / \mathrm{z}(\%): 881\left(5, \mathrm{M}^{+}-\mathrm{Me}\right), 839\left(100, \mathrm{M}^{+}-t \mathrm{Bu}\right)\right\}$. Weil trotz mehrfacher Kristallisationsversuche aus $n$-Hexan kein reines Produkt erhalten werden konnte, wurde diese Reaktion nicht weiter untersucht.

\subsubsection{Struktur von SiloxNa (12) im Festkörper}

Strukturbestimmungen an Alkalimetall-Silanolaten wurden vorwiegend an LithiumDerivaten ausgeführt. Unter ihnen dominieren tetramere Struktureinheiten mit $\mathrm{Li}_{4} \mathrm{O}_{4}$ Heterocuban-Gerüst [73-76], daneben sind auch trimere [77], dimere [78,79] und ein monomeres Beispiel beschrieben [80]. Seltener findet man die $\mathrm{K}_{4} \mathrm{O}_{4}$-Struktureinheit [79, 81, 82]. $\mathrm{Na}_{4} \mathrm{O}_{4}$-Heterocubangerüste weisen die Verbindungen $\left[\mathrm{NaOSiMe}_{3}\left\{\mathrm{OP}\left(\mathrm{NMe}_{2}\right)_{3}\right\}\right]_{4} \times \mathrm{OP}\left(\mathrm{NMe}_{2}\right)_{3}[83]$ und $\mathrm{Na}_{4}\left(\mathrm{OSiPh}_{3}\right)_{4}\left(\mathrm{H}_{2} \mathrm{O}\right)_{3}$ [84] auf. Ein weiterer Vertreter dieser Verbindungsklasse ist das Natriumsalz des Siloxliganden. Von Verbindung 12 konnten Kristalle für eine röntgenstrukturelle Untersuchung aus $n$-Hexan bei $-30{ }^{\circ} \mathrm{C}$ gewonnen werden. 


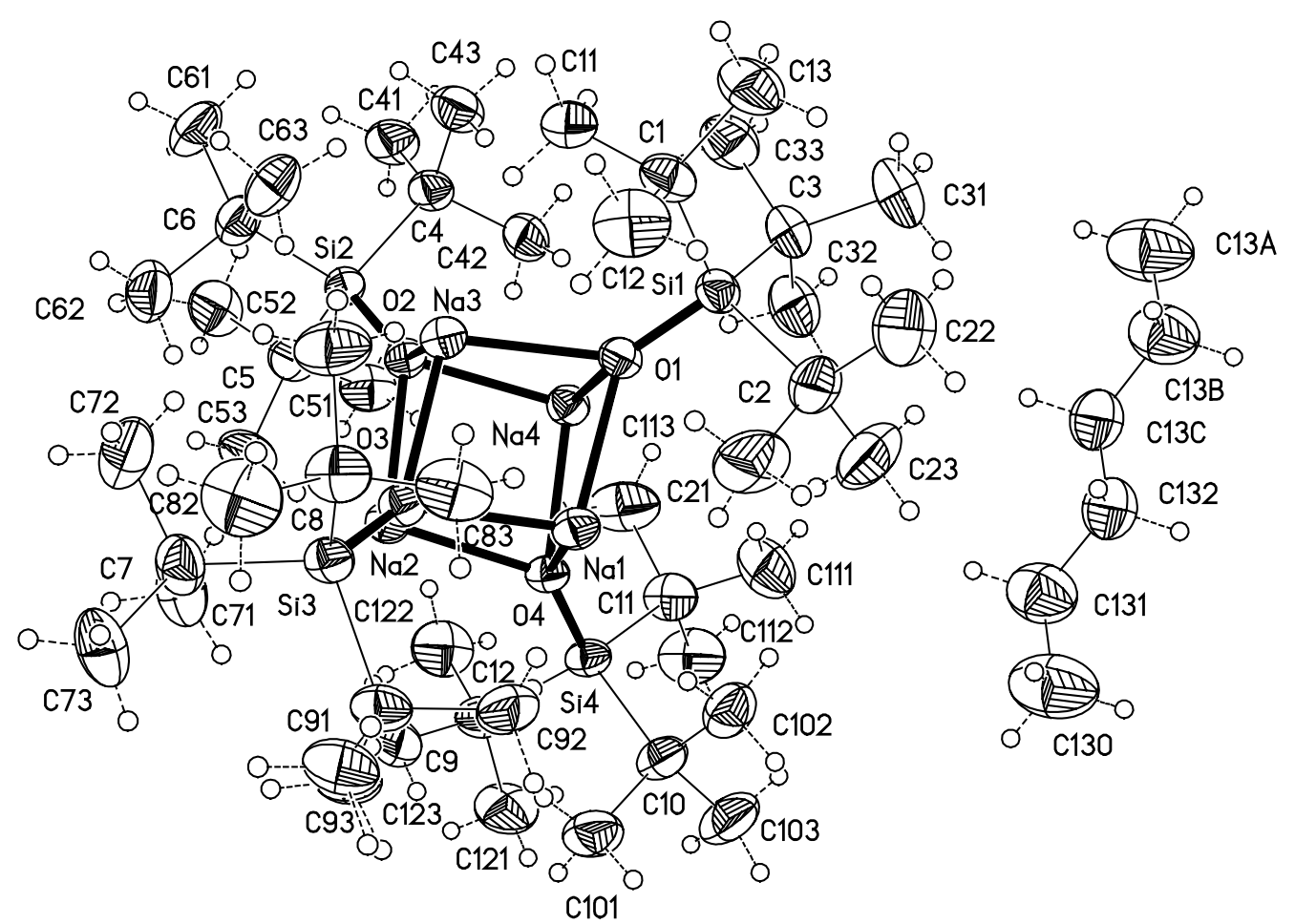

Abbildung 6: Struktur von NaSilox 12 im Festkörper

Tabelle 7: Charakteristische Bindungslängen (pm) und -Winkel $\left({ }^{\circ}\right)$ von 12

$\begin{array}{ll}\mathrm{Na}(1)-\mathrm{O}(1) & 231.31(2) \\ \mathrm{Na}(1)-\mathrm{O}(3) & 230.2(2) \\ \mathrm{Na}(1)-\mathrm{O}(4) & 229.2(2) \\ \mathrm{Si}(1)-\mathrm{O}(1) & 161.6(2) \\ \mathrm{Si}(1)-\mathrm{C}(1) & 194.4(3)\end{array}$

$\begin{array}{ll}\mathrm{O}(4)-\mathrm{Na}(1)-\mathrm{O}(3) & 97.01(6) \\ \mathrm{O}(3)-\mathrm{Na}(1)-\mathrm{O}(1) & 98.58(6) \\ \mathrm{O}(4)-\mathrm{Na}(1)-\mathrm{O}(1) & 99.52(6) \\ \mathrm{Na}(4)-\mathrm{O}(1)-\mathrm{Na}(3) & 81.57(6) \\ \mathrm{Na}(3)-\mathrm{O}(1)-\mathrm{Na}(1) & 80.72(6) \\ \mathrm{Na}(4)-\mathrm{O}(1)-\mathrm{Na}(1) & 80.20(6)\end{array}$

Ein Vergleich der Bindungslängen der dreifach koordinierten Natriumatome zu Sauerstoff zeigt eine Verlängerung in 12 gegenüber $\mathrm{Na}_{4}\left(\mathrm{OSiPh}_{3}\right)_{4}\left(\mathrm{H}_{2} \mathrm{O}\right)_{3}$ um 4 pm. Für die Bindungsverlängerung ist wahrscheinlich ein größerer sterischer Anspruch des Siloxliganden gegenüber der $\mathrm{OSiPh}_{3}$-Gruppe verantwortlich. Der hohe Platzbedarf der Siloxgruppe wird durch die vergleichsweise starke Verzerrung der Würfelstruktur in $\mathbf{1 2}$ deutlich. Findet man in 
$\mathrm{Na}_{4}\left(\mathrm{OSiPh}_{3}\right)_{4}\left(\mathrm{H}_{2} \mathrm{O}\right)_{3} \mathrm{O}-\mathrm{Na}-\mathrm{O}-$ und $\mathrm{Na}-\mathrm{O}-\mathrm{Na}-\mathrm{W}$ inkel von $91.3^{\circ}$ bis $94.1^{\circ}$ bzw. $85.0^{\circ}$ bis $87.2^{\circ}$, so liegen entsprechende Winkel in $\mathbf{1 2}$ bei $98.6^{\circ}$ bis $99.5^{\circ}$ bzw. $80.2^{\circ}$ bis $81.6^{\circ}$.

\subsubsection{Vergleich der NMR-Daten der neuen Bis-substituierten Verbindungen}

Tabelle 8: Übersicht der ${ }^{1} \mathrm{H},{ }^{13} \mathrm{C},{ }^{19} \mathrm{~F}$ und ${ }^{29} \mathrm{Si}-\mathrm{NMR}-\mathrm{Daten}$ der neuen Bis-substituierten Verbindungen

$\begin{array}{lcccc}\text { Verbindung } & \delta^{1} \mathrm{H}-\mathrm{NMR} & \delta^{13} \mathrm{C}-\mathrm{NMR} & \delta^{19} \mathrm{~F}-\mathrm{NMR} & \delta^{29} \mathrm{Si}-\mathrm{NMR} \\ \mathrm{BisTaF}_{4} & 0.28 / 3.09 & 2.5 / 110.7 & 81.5 & 8.1 \\ \mathrm{BisTaCl}_{4} & 0.45 / 4.65 & 4.2 / 146.3 & & 6.0 \\ \mathrm{BisTaBr}_{4} & 0.52 / 4.45 & & & 2.5 \\ \mathrm{BisNbF}_{4} & 0.31 / 4.65 & & 126.0 & 10.1 \\ \mathrm{BisNbCl}_{4} & 0.49 / 6.20 & 3.8 / 170.7 & & 9.4 \\ \mathrm{BisNbBr}_{4} & 0.53 / 5.99 & & & 5.5 \\ \mathrm{BisTiCl}_{3} & 0.38 / 6.29 & & & 3.4\end{array}$

Bei den ${ }^{1} \mathrm{H}-\mathrm{NMR}-$ Spektren erwartet man eine Tieffeldverschiebung für Protonen, die an elektronenziehende Substituenten tragende Kohlenstoffatome gebunden sind [85]. Die chemische Verschiebung der Protonen des CH-Fragments nimmt in der Reihe $\mathrm{MF}<<\mathrm{MBr}<\mathrm{MCl}$ zu. Aufgrund der starken Tendenz des Fluors, Rückbindungen auszubilden, ist es nicht verwunderlich, daß die Signale der Metallfluoride hochfeldverschoben sind. Das Bromid liefert mehr Ladungsdichte an das Metallzentrum als das Chlorid, was man auf eine bessere Polarisierbarkeit des Bromids zurückführen kann. Ein Vergleich der drei Chloride zeigt für die chemischen Verschiebungen der Protonen des C-H-Fragments eine Reihenfolge der Metalle $\mathrm{Ti}>\mathrm{Nb}>>\mathrm{Ta}$. Die ${ }^{19} \mathrm{~F}$ - und ${ }^{13} \mathrm{C}$-NMR-Spektren bestätigen diese Tendenz. 


\subsection{Einsatz der neuen Fluorierungsmittel $\mathrm{Ph}_{2} \mathrm{PbF}_{2}(13)$ und $\mathrm{Ph}_{3} \mathrm{BiF}_{2}(14)$}

\subsubsection{Vergleich der Eigenschaften von $\mathrm{Ph}_{2} \mathrm{PbF}_{2}(13)$ und $\mathrm{Ph}_{3} \mathrm{BiF}_{2}$ (14) mit $\mathrm{Me}_{3} \mathrm{SnF}$ (9)}

Wie einleitend bereits erwähnt, hat das $\mathrm{Me}_{3} \mathrm{SnF}$ neben den Vorteilen bei der Versuchsführung auch einige Nachteile. Späte Übergangsmetalle lassen sich nicht fluorieren und sterisch wenig anspruchsvolle Alkylliganden werden substituiert. Aufgrund der gleichen Hauptgruppenzugehörigkeit können für Zinn- und Bleiverbindungen ähnliche chemische Eigenschaften angenommen werden. Zwischen Zinn und Bismut besteht eine Schrägbeziehung, die auf Ähnlichkeiten im chemischen Verhalten schließen läßt. Nach dem Pearson-Konzept $[86,87]$ der harten und weichen Säuren und Basen werden Blei(IV) und Bismut(V) als mittelhart angesehen, während Fluorid eindeutig zu den harten Basen zählt. Das Pearson-Konzept geht davon aus, daß gleich geartete Säuren und Basen harmonieren. Aus diesem Grund sollten sowohl Bismut als auch Blei ein stärkeres Bestreben zeigen, das Fluorid durch Chlorid zu ersetzen als das Zinn.

$\mathrm{Ph}_{3} \mathrm{BiCl}_{2}$ ist eine gegenüber Hitze, Wasser und Sauerstoff robuste Verbindung, die kein Bestreben zeigt, weitere Halogene gegen Phenylgruppen auszutauschen. $\mathrm{Ph}_{4} \mathrm{BiCl}$ und $\mathrm{Ph}_{5} \mathrm{Bi}$ lassen sich zwar aus $\mathrm{Ph}_{3} \mathrm{BiCl}_{2}$ und $\mathrm{PhLi}$ darstellen, sind aber bei Raumtemperatur nicht stabil $[88,89]$. Daraus läßt sich ableiten, daß $\mathrm{Ph}_{3} \mathrm{BiF}_{2}$ vermutlich keine Alkylgruppen durch Fluor ersetzen wird.

Verbindung 13 zeigt eine geringe Löslichkeit in allen gängigen organischen Lösungsmitteln, wohingegen 14 sehr gut löslich ist. Aus $n$-Hexan konnten bei $-30{ }^{\circ} \mathrm{C}$ Kristalle von 14 gewonnen werden, die für eine röntgenstrukturelle Untersuchung geeignet waren. Abbildung 7 zeigt die Struktur von Verbindung 14 im Festkörper und in Tabelle 9 sind charakteristische Bindungslängen und -winkel aufgeführt. Verbindung 14 kristallisiert monoklin in der Raumgruppe $C 2 / c$ mit 8 Molekülen pro Basiseinheit und liegt im Kristall monomer vor. Das Metallatom ist trigonal-bipyramidal koordiniert. Die Phenylgruppen befinden sich in der Äquatorialebene und die Fluoratome nehmen axiale Positionen ein. 


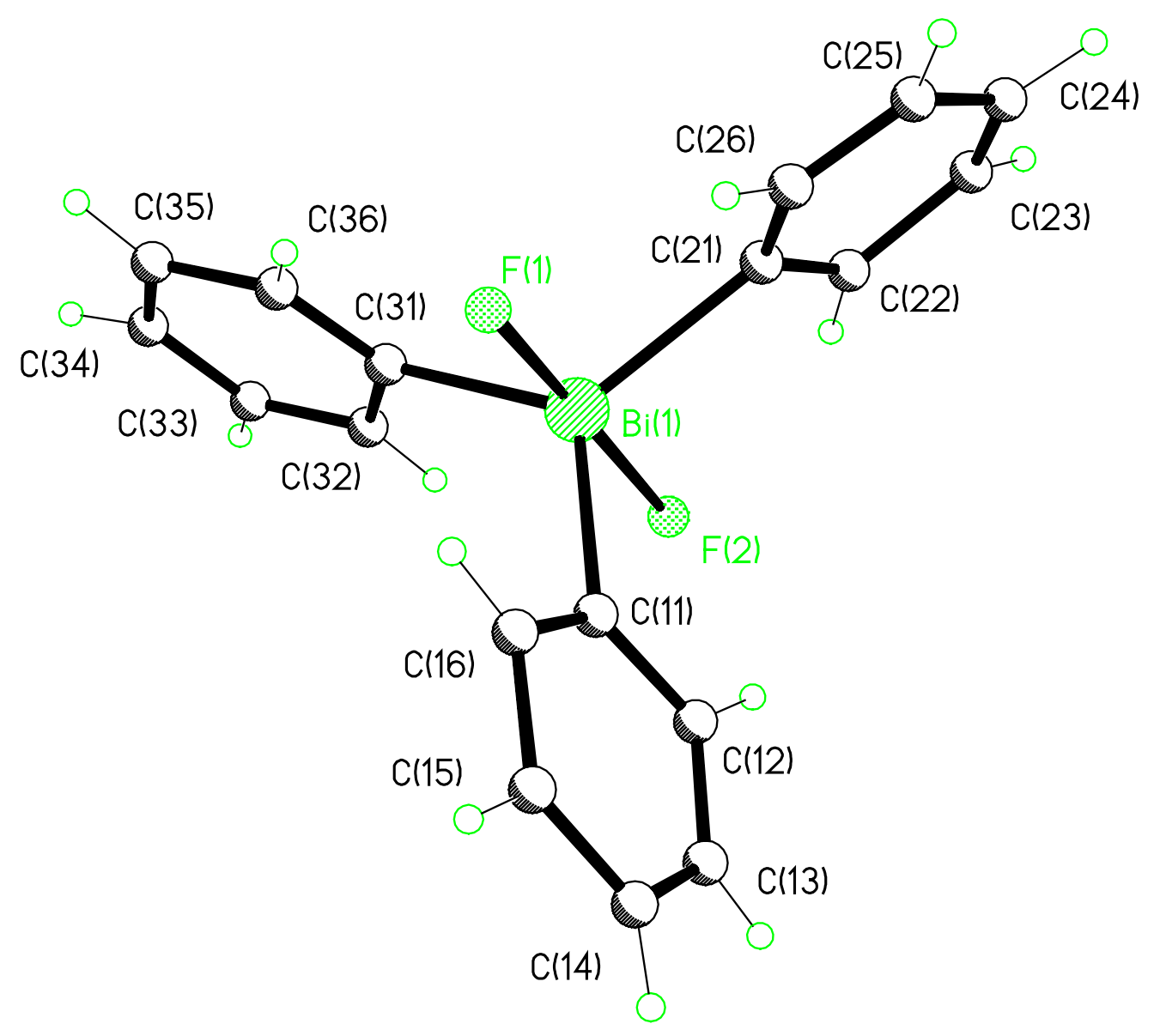

Abbildung 7: Struktur von Verbindung 14 im Festkörper

Tabelle 9: Charakteristische Bindungslängen (pm) und -winkel $\left(^{\circ}\right)$ von Verbindung 14

$\begin{array}{ll}\operatorname{Bi}(1)-F(1) & 211 .(2) \\ \operatorname{Bi}(1)-F(2) & 236.0(14) \\ \operatorname{Bi}(1)-C(11) & 218 .(3) \\ \operatorname{Bi}(1)-C(21) & 221 .(2) \\ \operatorname{Bi}(1)-C(31) & 216 .(3)\end{array}$

$\begin{array}{ll}\mathrm{F}(1)-\operatorname{Bi}(1)-\mathrm{F}(2) & 177.0(5) \\ \mathrm{F}(1)-\operatorname{Bi}(1)-\mathrm{C}(11) & 87.7(8) \\ \mathrm{F}(1)-\operatorname{Bi}(1)-\mathrm{C}(21) & 91.1(9) \\ \mathrm{F}(1)-\operatorname{Bi}(1)-\mathrm{C}(31) & 89.1(8) \\ \mathrm{C}(11)-\operatorname{Bi}(1)-\mathrm{C}(21) & 115.9(9)\end{array}$




\subsubsection{Darstellung von $\mathrm{Cp}^{*} \mathrm{TiF}_{3}, \mathrm{Cp}_{2} \mathrm{ZrF}_{2}$ undC $\mathrm{p}_{2} \mathrm{HfF}_{2}$ unter Verwendung von 13 und 14}

Als nächstes soll die metathetische Aktivität von 13 und 14 anhand analoger Reaktionen überprüft werden, die bereits mit $\mathrm{Me}_{3} \mathrm{SnF}$ durchgeführt wurden. $\mathrm{Zu}$ diesem $\mathrm{Zweck}$ wurden die Komplexe $\mathrm{Cp}^{*} \mathrm{TiCl}_{3}, \mathrm{Cp}_{2} \mathrm{ZrCl}_{2}$ und $\mathrm{Cp}_{2} \mathrm{HfCl}_{2}$ mit 13 und 14 fluoriert. Die Reaktionen wurden in $\mathrm{CH}_{2} \mathrm{Cl}_{2}$ durchgeführt. Nach 12 stündigem Rühren bei Raumtemperatur und Abtrennung des Lösungsmittels konnten die Zielverbindungen mittels Sublimation isoliert werden.

$$
\begin{gathered}
2 \mathrm{Cp}^{*} \mathrm{TiCl}_{3}+3\left(\mathrm{M}^{*}\right) \mathrm{F}_{2} \longrightarrow 2 \mathrm{Cp}^{*} \mathrm{TiF}_{3}+3\left(\mathrm{M}^{*}\right) \mathrm{Cl}_{2} \\
\mathrm{Cp}_{2} \mathrm{MCl}_{2}+\left(\mathrm{M}^{*}\right) \mathrm{F}_{2} \longrightarrow \mathrm{Cp}_{2} \mathrm{MF}_{2}+\left(\mathrm{M}^{*}\right) \mathrm{Cl}_{2} \\
\mathrm{M}=\mathrm{Zr}, \mathrm{Hf} ;\left(\mathrm{M}^{*}\right)=\mathrm{Ph}_{3} \mathrm{Bi}, \mathrm{Ph}_{2} \mathrm{~Pb}
\end{gathered}
$$

Die auf diesem Weg dargestellten Fluorokomplexe waren frei von Verunreinigungen. In Tabelle 10 werden die Ausbeuten der drei obigen Reaktionen unter Verwendung der Fluorierungsmittel $\mathrm{Me}_{3} \mathrm{SnF}$, (7) $\mathrm{Ph}_{2} \mathrm{PbF}_{2}$ (13) und $\mathrm{Ph}_{3} \mathrm{BiF}_{2}$ (14) gegenübergestellt. Es fällt auf, daß in allen drei Fällen 7 die besten Ausbeuten liefert. Eine Begründung findet man in der leichten Abtrennung des Beiprodukts $\mathrm{Me}_{3} \mathrm{SnCl}$, das im Vakuum abkondensiert werden kann, wohingegen die Beiprodukte $\mathrm{Ph}_{2} \mathrm{PbCl}_{2}$ und $\mathrm{Ph}_{3} \mathrm{BiCl}_{2}$ bei 0.02 mbar nicht sublimierbar sind. Die Trennung beruht in diesen Fällen darauf, daß das Produkt aus der Mischung heraussublimiert wird. Dieses Verfahren wirkt sich ungünstig auf die Ausbeute aus.

Tabelle 10: Vergleich der Ausbeuten (\%) von $\mathrm{Cp}^{*} \mathrm{TiF}_{3}, \mathrm{Cp}_{2} \mathrm{ZrF}_{2}$ und $\mathrm{Cp}_{2} \mathrm{HfF}_{2}$ unter Verwendung der Fluorierungsmittel 7, 13 und 14

$\begin{array}{cccc}\text { Produkt/Fluorierungsmittel } & \mathrm{Me}_{3} \mathrm{SnF} & \mathrm{Ph}_{2} \mathrm{PbF}_{2} & \mathrm{Ph}_{3} \mathrm{BiF}_{2} \\ \mathrm{Cp}^{*} \mathrm{TiF}_{3} & 92 & 65 & 72 \\ \mathrm{Cp}_{2} \mathrm{ZrF}_{2} & 73 & 27 & 40 \\ \mathrm{Cp}_{2} \mathrm{HfF}_{2} & 92 & 23 & 44\end{array}$




\subsubsection{Darstellung von $\mathrm{Cp}^{9} \mathrm{Cp}^{*} \mathrm{HfCl}_{2}$ (16)}

Nachdem die Fluoridübertragung von 13 und 14 anhand literaturbekannter Verbindungen getestet wurden, sollten als nächstes mit diesen Fluorierungsmitteln neue Verbindungen hergestellt werden. Die Fluorokomplexe sollen eine gute Löslichkeit in Kohlenwasserstoffen wie $n$-Hexan aufweisen. Zu diesem Zweck wurden Metallocene der 4. Gruppe ausgewählt, die unterschiedlich substituierte Cp-Ringe tragen. Als modifizierte CpRinge wurden $\mathrm{Cp}^{*} \quad\left(\mathrm{Cp}^{*}=\mathrm{C}_{5} \mathrm{Me}_{5}\right)$ und $\mathrm{Cp}^{\prime} \quad\left(\mathrm{Cp}^{\prime}=\mathrm{C}_{5} \mathrm{H}_{4} \mathrm{SiMe}_{3}\right)$ verwendet. Als Ausgangsverbindungen dienten die Chlorokomplexe $\mathrm{Cp} * \mathrm{Cp}^{\prime} \mathrm{MCl}_{2}(\mathrm{M}=\mathrm{Zr}(\mathbf{1 5}), \mathrm{Hf}(\mathbf{1 6}))$. Die Zirconiumverbindung wurde bereits von Tilley et al. synthetisiert [90]. Dieser Synthesestrategie folgend kann aus $\mathrm{Cp}^{*} \mathrm{HfCl}_{3}$ und $\mathrm{Cp}$ 'Li in THF als Lösungsmittel das unsymmetrische Hafnocendichlorid 16 hergestellt werden.

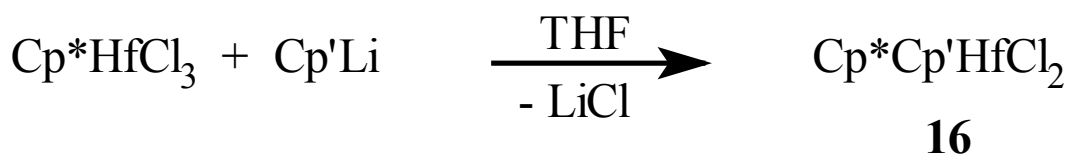

Verbindung 16 wurde spektroskopisch untersucht. Das lösungsmittelfreie Produkt liefert korrekte Elementaranalysen. Das ${ }^{1} \mathrm{H}-\mathrm{NMR}$-Spektrum in $\mathrm{CDCl}_{3}$ zeigt die erwarteten Signale im korrekten Intensitätsverhältnis. Das Signal der $\mathrm{SiMe}_{3}$-Protonen zeigt sich bei $\delta 0.30 \mathrm{ppm}$. Das Singulett der Protonen des Cp*-Rings findet man bei $\delta 2.08 \mathrm{ppm}$. Die 4 Protonen am $\mathrm{C}_{5} \mathrm{H}_{4} \mathrm{SiMe}_{3}$-Ring erzeugen zwei Tripletts bei $\delta 6.07$ und $6.40 \mathrm{ppm}$ mit einer ${ }^{3} J_{\mathrm{H}-\mathrm{H}}$-Kopplung von $2.6 \mathrm{~Hz}$. Massenspektroskopisch (EI) lassen sich der Molekülpeak m/z 522 (25\%) und das Fragment $\mathrm{M}^{+}-\mathrm{Me}$ m/z 507 (100\%) nachweisen.

\subsubsection{Versuch der Monofluorierung von $\mathrm{Cp}^{*} \mathrm{Cp}^{\prime} \mathrm{MCl}_{2}(\mathrm{M}=\mathrm{Zr}$, $\mathrm{Hf})$ mit 13}

Wie bereits unter 2.3.2. gezeigt, gestaltet sich die Abtrennung der Beiprodukte $\mathrm{Ph}_{3} \mathrm{BiCl}_{2}$ und $\mathrm{Ph}_{2} \mathrm{PbCl}_{2}$ problematisch und führt zu einer Verminderung der Ausbeute. Bei einer Fluorierung mit 13 kann man sich zunutze machen, daß das entsprechende Beiprodukt in n-Hexan so gut wie nicht löslich ist: wählt man eine gut lösliche metallorganische Komponente, ist das Beiprodukt einfach abzufiltrieren. 
Bei der Katalysatorforschung für die Olefinpolymerisation hat sich gezeigt, daß Fluorokomplexe der 4. Gruppe schon bei kleinem Cokatalysator-Katalysator Verhältnis ihre maximale Aktivität entwickeln. Die Ausgangsverbindungen sind bereits synthetisiert worden, doch entsprechende Versuche mit gemischt halogenierten Systemen wurden bislang nicht durchgeführt. Bei Metallocenen der 4. Gruppe gelingt die Monofluorierung durch Methathese der Chloride mit einem Äquivalent $\mathrm{Me}_{3} \mathrm{SnF}$ [91]. Gemischthalogenierte, nur einen Cp-Ring tragende Spezies der 4. Gruppe lassen sich nicht nach der oben beschriebenen Route darstellen. Hier wird zuerst mit drei Äquivalenten $\mathrm{Me}_{3} \mathrm{SnF}$ vollständig fluoriert und nachfolgend Fluorid durch Chlorid oder Bromid mit Hilfe von $\mathrm{Me}_{3} \mathrm{SiX}(\mathrm{X}=\mathrm{Cl}, \mathrm{Br})$ ersetzt [92]. Ein Fluor-Chlor Austausch mit $\mathrm{Me}_{3} \mathrm{SiCl}$ wurde bereits früher bei der Herstellung von Silylhydrazin-Ringsystemen beschrieben [93, 94]. Iodide lassen sich auf keinem der genannten Wege darstellen [95].

Im folgenden soll untersucht werden, ob es möglich ist, mit 13 eine Monofluorierung vorzunehmen. Dafür wurde $\mathrm{Cp}^{*} \mathrm{Cp}^{\prime} \mathrm{MCl}_{2}\left(\mathrm{M}=\mathrm{Zr}\right.$, Hf) mit einem halben Äquivalent $\mathrm{Ph}_{2} \mathrm{PbF}_{2}$ umgesetzt.

$$
4 \mathrm{Cp}^{*} \mathrm{Cp}^{\prime} \mathrm{MCl}_{2}+2 \mathrm{Ph}_{2} \mathrm{PbF}_{2}
$$

$$
\mathrm{Cp} * \mathrm{Cp}^{\prime} \mathrm{MCl}_{2}+\mathrm{Cp} * \mathrm{Cp} \mathrm{M}(\mathrm{Cl}) \mathrm{F}+\mathrm{Cp} * \mathrm{Cp}^{\prime} \mathrm{MF}_{2}
$$

$$
\mathrm{M}=\mathrm{Zr}, \mathrm{Hf}
$$

$$
\text { : }
$$

2
1

Es wurde die Bildung von Produktgemischen aus Edukt, gemischt halogenierten und zweifach fluorierten Verbindungen in einem molaren Verhältnis von $1: 2: 1$ beobachtet. Die Elementaranalysen liefern Werte, die auf das gemischt halogenierte Produkt hinweisen, weil Edukt und zweifach substituertes Produkt in gleichem Verhältnis vorliegen. Betrachtet man die ${ }^{1}$ H-NMR-Spektren, so wird deutlich, daß Produktgemische entstanden sind. Man findet für die Protonen der $\mathrm{SiMe}_{3}$-Gruppe drei Singuletts bei $\delta 0.07,0.21$ und $0.26(\mathrm{Zr})$ und 0.21 , 0.26 und 0.30 (Hf) ppm in einem Intensitätsverhältnis von jeweils $1: 2: 1$. Die Signale der Difluoride sind am stärksten hochfeldverschoben. Das gleiche Bild zeigt sich für die Protonen der Cp*-Gruppe, deren Signale bei $\delta 1.96,1.98$ und 2.00 (Zr) bzw. 1.99, 2.02 und 2.05 (Hf) ppm beobachtet werden. Aufgrund einer ${ }^{4} J_{\mathrm{H}-\mathrm{F}}-$ Kopplung ergeben die Protonen der $\mathrm{Cp}^{*}-$ 
Gruppen der gemischten Halogenide Dubletts und die der Difluoride Tripletts mit ${ }^{4} J_{\mathrm{F}-\mathrm{H}}-$ Kopplungskonstanten von jeweils $0.8 \mathrm{~Hz}(\mathrm{Zr})$ bzw. $0.6 \mathrm{~Hz}(\mathrm{Hf})$. Die 4 Protonen des Cp'-Rings liefern für die Dichloride zwei Tripletts bei $\delta 6.08$ und 6.45 (Zr) bzw. 6.07 und 6.40 (Hf) ppm mit einer ${ }^{3} J_{\mathrm{H}-\mathrm{H}}-$ Kopplung von $2.6 \mathrm{~Hz}$. Die gemischten Halogenide zeigen zwei Dubletts vom Triplett bei $\delta 6.02$ und 6.61 (Zr) bzw. 5.98 und 6.53 (Hf) ppm mit ${ }^{3} J_{\mathrm{H}-\mathrm{H}^{-}}$und ${ }^{3} J_{\mathrm{H}-\mathrm{F}}-$ Kopplungen von 2.8 und $3.0 \mathrm{~Hz}(\mathrm{Zr})$ bzw. jeweils $2.8 \mathrm{~Hz}(\mathrm{Hf})$. Die Difluoride ergeben schließlich zwei Multipletts bei $\delta 6.23$ und 6.32 (Zr) bzw. 6.14 und 6.30 (Hf) ppm. In den ${ }^{19}$ F-NMR-Spektren findet man Singuletts für die gemischt halogenierten Verbindungen bei $\delta$ 49.3 (Zr) bzw. 7.8 (Hf) ppm und für die Bifluoride bei $\delta 14.7$ (Zr) bzw. -22.6 (Hf) ppm.

\subsubsection{Struktur von $\mathrm{Cp}^{*} \mathrm{Cp}^{\prime} \mathrm{ZrCl}_{2}(15)$ im Festkörper}

Das Produktgemisch aus $\mathrm{Cp}^{*} \mathrm{Cp}^{\prime} \mathrm{ZrCl}_{2}, \mathrm{Cp}^{*} \mathrm{Cp} \mathrm{Z}^{\prime} \mathrm{Zr}(\mathrm{Cl}) \mathrm{F}$ und $\mathrm{Cp}^{*} \mathrm{Cp}^{\prime} \mathrm{ZrF}_{2}$ wurde bei $-30{ }^{\circ} \mathrm{C}$ aus einem Toluol/Hexan-Lösungsmittelgemisch $(1: 1)$ kristallisiert. Aufgrund der geringeren Löslichkeit der Chloride gegenüber den Fluoriden in Kohlenwasserstoffen kristallisierte 15 zuerst und wurde röntgenstrukturell charakterisiert. Verbindung 15 kristallisiert in der orthorhombischen Raumgruppe $P 2{ }_{1} 2_{1} 2_{1}$. Abbildung 8 zeigt die Struktur von Verbindung 15 im Festkörper und in Tabelle 11 sind wichtige Bindungslängen und -winkel aufgeführt. 


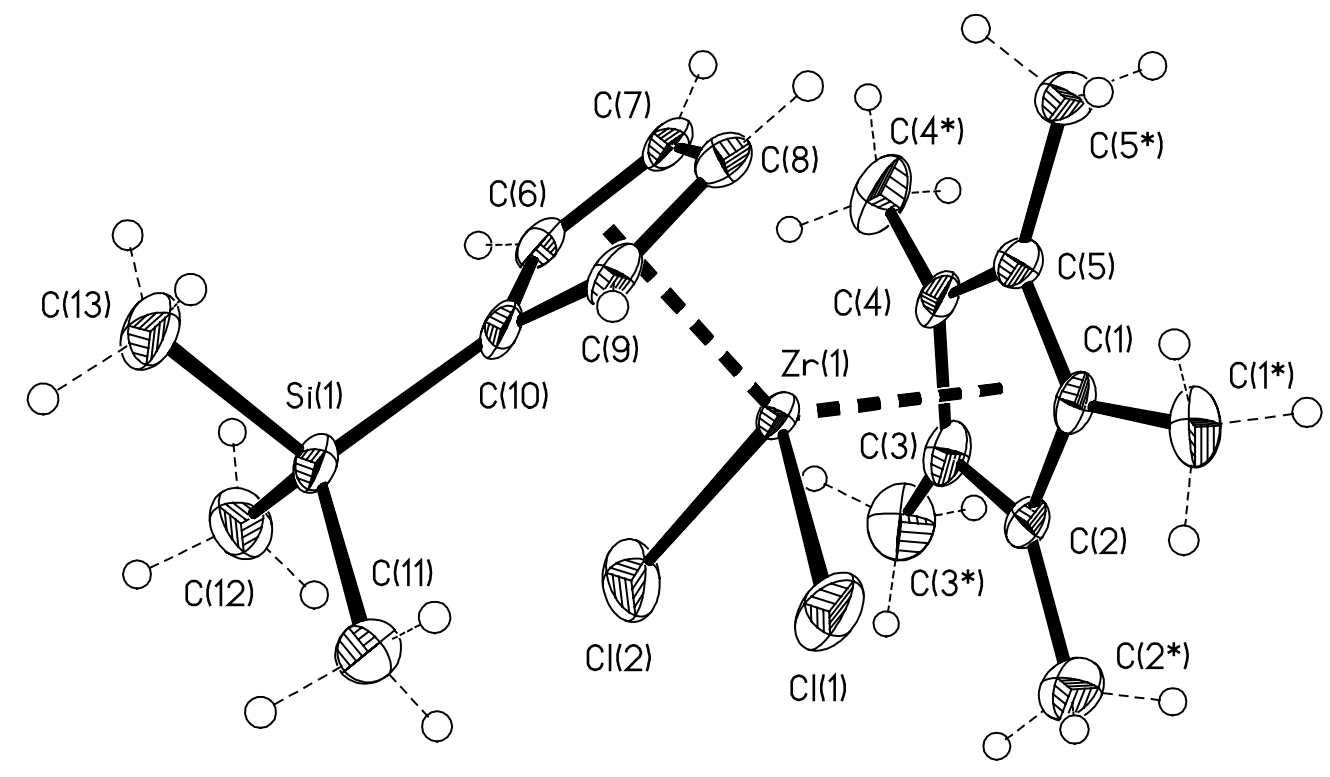

Abbildung 8: Molekülstruktur von 15 im Kristall

Tabelle 11: Wichtige Bindungslängen $(\mathrm{pm})$ und -winkel $\left(^{\circ}\right)$ von 15

$\begin{array}{ll}\mathrm{Zr}(1)-\mathrm{Cl}(1) & 2.4455(14) \\ \mathrm{Zr}(1)-\mathrm{Cl}(2) & 2.408(2) \\ \mathrm{Zr}(1)-\mathrm{C}\left(\mathrm{Cp}^{*}\right) & 2.530(3) \\ \mathrm{Zr}(1)-\mathrm{C}\left(\mathrm{Cp}^{\prime}\right) & 2.526(8)\end{array}$

Wie andere Zirconocene zeigt Verbindung 15 eine monomere Struktur im Festkörper. Aus dem großen Platzbedarf der substituierten Cp-Ringe resultiert eine verzerrt tetraedrische Geometrie um das Metallatom. In der mit 15 vergleichbaren Verbindung $\mathrm{C}_{5} \mathrm{H}_{4}\left(\mathrm{SiMe}_{3}\right) \mathrm{C}_{5} \mathrm{H}_{3}\left(\mathrm{SiMe}_{3}\right)_{2} \mathrm{ZrCl}_{2}$ nehmen die $\mathrm{Zr}$-Cl-Bindungsabstände (243.9(1) pm) und ClZr-Cl-Bindungswinkel $\left(95.8(1)^{\circ}\right)$ ähnliche Werte an [96]. 


\subsubsection{Darstellung von $\mathrm{Cp}^{*} \mathrm{Cp}^{\prime} \mathrm{ZrF}_{2}$ (17) und $\mathrm{Cp}^{*} \mathrm{Cp}^{\prime} \mathrm{HfF}_{2}$ (18)}

Für die Darstellung der Difluoride 17 und 18 wurden die Chloride $\mathrm{Cp}^{*} \mathrm{Cp}^{\prime} \mathrm{MCl}_{2}(\mathrm{M}=$ $\mathrm{Zr}$, Hf) mit einem Äquivalent $\mathrm{Ph}_{2} \mathrm{PbF}_{2}$ (13) in $\mathrm{CH}_{2} \mathrm{Cl}_{2}$ bei Raumtemperatur zur Reaktion gebracht. Das $\mathrm{CH}_{2} \mathrm{Cl}_{2}$ wurde im Vakuum abgetrennt und das Produkt in $n$-Hexan aufgenommen. Da das Beiprodukt $\mathrm{Ph}_{2} \mathrm{PbCl}_{2}$ in $n$-Hexan nahezu unlöslich ist, konnte es abfiltriert werden. Durch Umkristallisation aus $n$-Hexan gelang die Reinigung des Produkts. Aufgrund der im Vergleich zu 2.3.2. günstigeren Aufarbeitung kann das Produkt hier in hoher Ausbeute isoliert werden.

$$
\begin{array}{lcl}
\mathrm{Cp}^{*} \mathrm{Cp}^{\prime} \mathrm{MCl}_{2}+\mathrm{Ph}_{2} \mathrm{PbF}_{2} \longrightarrow & \mathrm{Cp}^{*} \mathrm{Cp}^{\prime} \mathrm{MF}_{2}+\mathrm{Ph}_{2} \mathrm{PbCl}_{2} \\
\mathbf{1 5}: \mathrm{M}=\mathrm{Zr} & & \mathbf{1 7 :} \mathrm{M}=\mathrm{Zr} \\
\mathbf{1 6}: \mathrm{M}=\mathrm{Hf} & \mathbf{1 3} & \mathbf{1 8 :} \mathrm{M}=\mathrm{Hf}
\end{array}
$$

Die Verbindungen 17 und $\mathbf{1 8}$ sind farblose Feststoffe, die in allen gängigen organischen Lösungsmitteln gut löslich sind. Zusammensetzung und Konstitution von 17 und 18 wurden durch Elementaranalysen, NMR- und IR-Spektroskopie sowie Massenspektrometrie ermittelt.

Die chemischen Verschiebungen und Intensitätsverhältnisse der Signale der ${ }^{1} \mathrm{H}$ - und ${ }^{19}$ F-NMR-Spektren für 17 und 18 stimmen mit den unter 2.3.4. diskutierten überein.

Durch Umkristallisieren aus $n$-Hexan erhält man röntgenstrukturell vermeßbare Kristalle beider Verbindungen. Der Zirconiumkomplex kristallisiert in der monoklinen Raumgruppe $C 2 / c$. Der entsprechende Hafniumkomplex kristallisiert ebenfalls monoklin, aber in der Raumgruppe $P 2_{1} / c$. In Abbildung 9 ist die Festkörperstruktur von 18 dargestellt (für 17 gilt eine entsprechende Numerierung), wichtige Bindungslängen und -winkel sind in Tabelle 12 aufgeführt. 


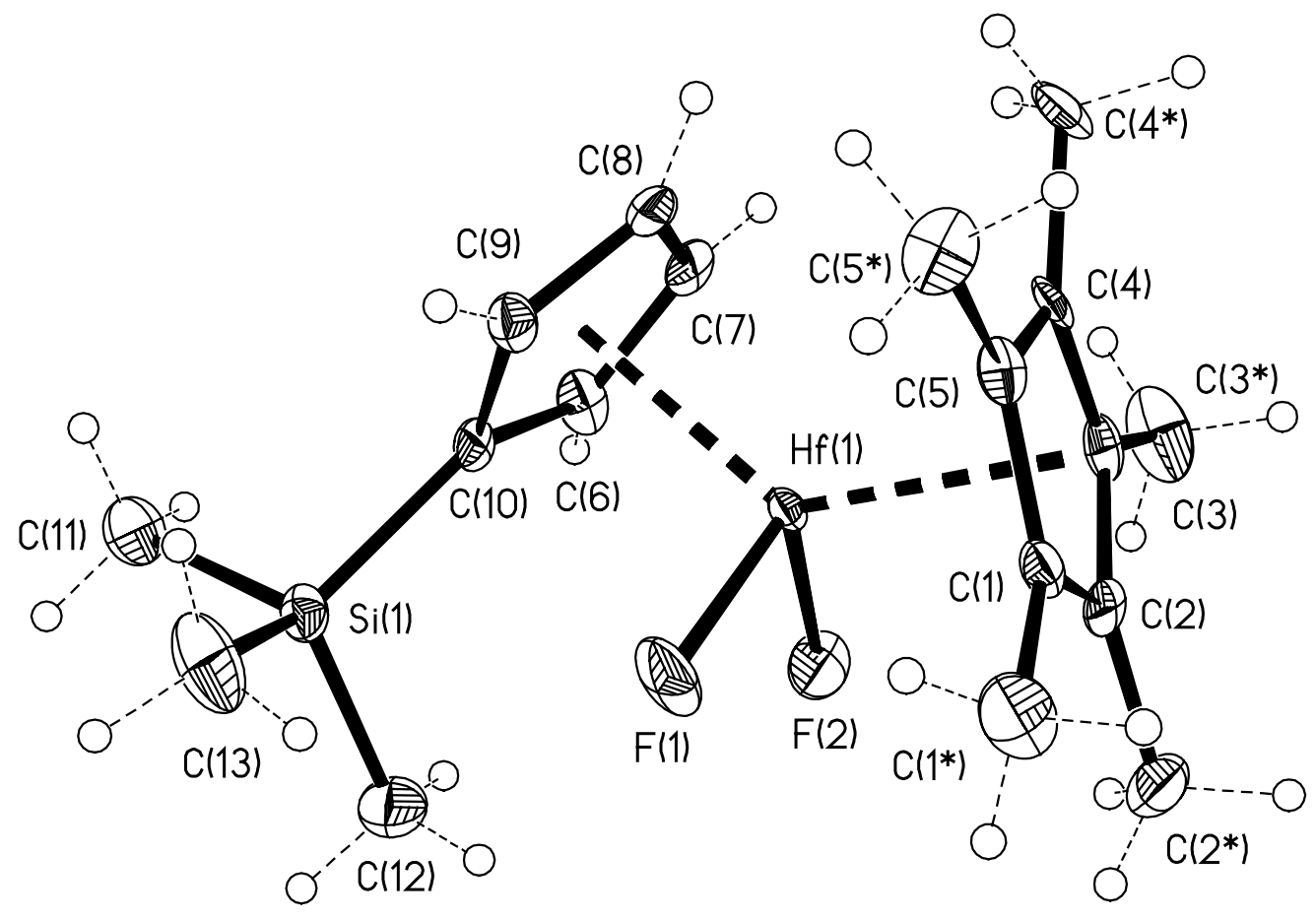

Abbildung 9: Molekülstruktur von 18 im Kristall

Tabelle 12: Charakteristische Bindungslängen (pm) und -winkel $\left(^{\circ}\right)$ von 17 und 18

$\begin{array}{llll}\operatorname{Zr}(1)-\mathrm{F}(1) & 1.962(2) & \operatorname{Hf}(1)-\mathrm{F}(1) & 1.935(6) \\ \mathrm{Zr}(1)-\mathrm{F}(2) & 1.968(2) & \operatorname{Hf}(1)-\mathrm{F}(2) & 1.945(6) \\ \mathrm{Zr}(1)-\mathrm{C}\left(\mathrm{Cp}^{*}\right) & 2.530(5) & \mathrm{Hf}(1)-\mathrm{C}\left(\mathrm{Cp}^{*}\right) & 2.506(7) \\ \mathrm{Zr}(1)-\mathrm{C}\left(\mathrm{Cp}^{\prime}\right) & 2.533(2) & \left.\mathrm{Hf}(1)-\mathrm{C}(\mathrm{Cp})^{\prime}\right) & 2.514(5) \\ \mathrm{F}(1)-\mathrm{Zr}(1)-\mathrm{F}(2) & 98.04(8) & \mathrm{F}(1)-\mathrm{Hf}(1)-\mathrm{F}(2) & 98.5(3)\end{array}$

Die Zirconocendifluoride $\mathrm{Cp}^{\prime}{ }_{2} \mathrm{ZrF}_{2}\left(\mathrm{Cp},{ }^{\prime}=\mathrm{C}_{5} \mathrm{H}_{3}\left(\mathrm{SiMe}_{3}\right)_{2}\right)$ [27] und $\mathrm{Cp}_{2} \mathrm{ZrF}_{2}$ [97] wurden röntgenstrukturell charakterisiert. Überraschend ist der große M-F-Bindungsabstand in $\mathrm{Cp}{ }^{\prime}{ }_{2} \mathrm{ZrF}_{2}$ mit 221.3 pm gegenüber $\mathrm{Cp}_{2} \mathrm{ZrF}_{2}$ (198 pm). Der Metall-Fluor-Bindungsabstand in 17 und 18 steht somit im Widerspruch zu dem in $\mathrm{Cp}{ }_{2}{ }_{2} \mathrm{ZrF}_{2}$, wenn man davon ausgeht, daß sich mit zunehmender Substitution des Cp-Rings der Halogenid-Metall-Abstand vergrößert. Vergleicht man die Halogenid-Metall-Halogenid-Bindungswinkel in $\mathrm{Cp}_{2} \mathrm{ZrF}_{2}\left(96^{\circ}\right.$ ) und $\mathrm{Cp}{ }_{2} \mathrm{ZrF}_{2}\left(100^{\circ}\right)$ mit denen von 17 und 18, so fällt auf, daß mit steigendem sterischem 
Anspruch des Cp-Rests der Bindungswinkel größer wird. Gleichzeitig läßt sich eine Vergrößerung des Cp-M-Cp-Winkels beobachten.

\subsubsection{Darstellung von $\left[\mathrm{Ph}_{3} \mathrm{BiCl}\right]^{+}\left[\mathrm{Cp} * \mathrm{TaF}_{5}\right]^{-}(19)$}

Im Folgenden soll überprüft werden, ob sich $\mathrm{Ph}_{3} \mathrm{BiF}_{2}$ für die Fluorierung von $\mathrm{Cp}^{*} \mathrm{TaCl}_{4}$ eignet. Dabei stand die Frage im Vordergrund, inwieweit sich Bismutfluoride überhaupt für Fluorierungen von organometallischen Komplexen der 5. Gruppe eignen.
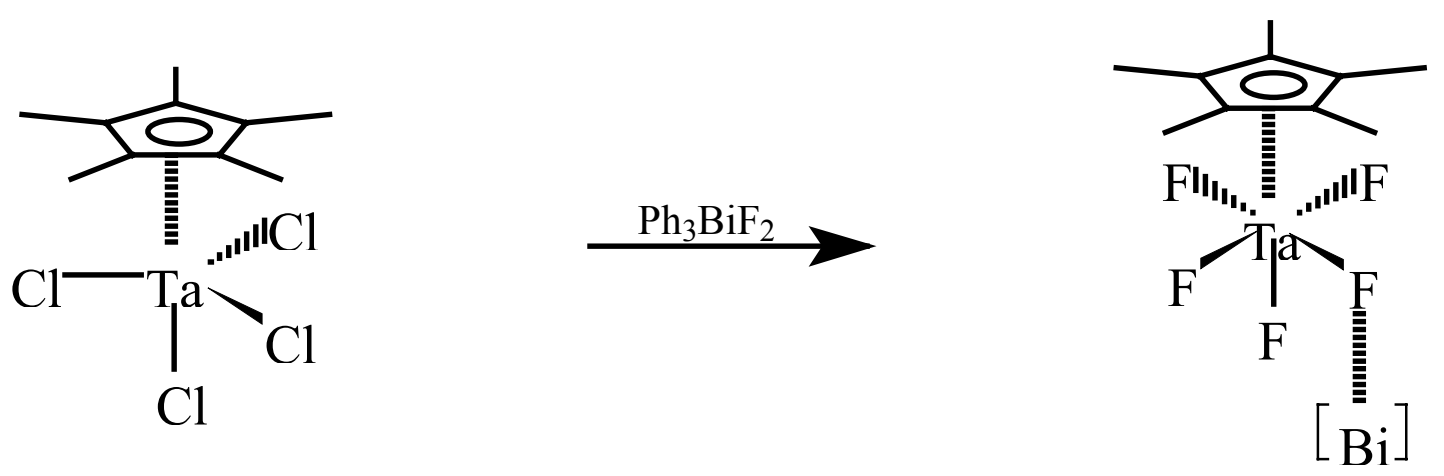

Das Reaktionsprodukt wurde NMR-spektroskopisch untersucht. Das ${ }^{1} \mathrm{H}-\mathrm{NMR}$ Spektrum zeigt ein Singulett bei $\delta 2.11 \mathrm{ppm}$, das von den Protonen des Cp* stammt und die Multipletts von den Protonen der drei Phenylgruppen. Im Vergleich dazu liefern die Protonen des $\mathrm{Cp}^{*}$ in $\mathrm{Cp}^{*} \mathrm{TaF}_{4}$ ein Singulett bei $\delta 1.99 \mathrm{ppm}$. Die ${ }^{19} \mathrm{~F}-\mathrm{NMR}-$ Spektren ähnelten einander überhaupt nicht, denn die Fluoratome von $\mathrm{Cp}^{*} \mathrm{TaF}_{4}$ ergeben ein breites Signal bei $\delta 18-22$ ppm. Das ${ }^{19}$ F-NMR-Spektrum des Reaktionsprodukts hingegen weist ein Quintett bei $\delta-83.3$ ppm und ein Dublett bei $\delta 26.6 \mathrm{ppm}$ mit gleichen Kopplungskonstanten auf. Es ähnelt vielmehr dem ${ }^{19} \mathrm{~F}-\mathrm{NMR}-$ Spektrum von $\left[\mathrm{Et}_{3} \mathrm{NH}\right]^{+}\left[\mathrm{Cp}^{*} \mathrm{TaF}_{5}\right]^{-}$, das ebenfalls ein Dublett der vier äquatorial positionierten Fluoratome und ein Quintett für das axiale Fluoratom zeigt [36].

$$
\begin{array}{ccc} 
& \mathrm{Ta}-\mathrm{F}_{\mathrm{eq}} & \mathrm{Ta}-\mathrm{F}_{\mathrm{ax}} \\
{\left[\mathrm{Et}_{3} \mathrm{NH}\right]^{+}\left[\mathrm{Cp}^{*} \mathrm{TaF}_{5}\right]^{-}} & \delta 12.7\left(\mathrm{~d},{ }^{2} J_{\mathrm{F}-\mathrm{F}}=90.5\right) & \delta=-16.2\left(\text { quintet, }{ }^{2} J_{\mathrm{F}-\mathrm{F}}=90.1\right) \\
{\left[\mathrm{Ph}_{3} \mathrm{BiCl}\right]^{+}\left[\mathrm{Cp}^{*} \mathrm{TaF}_{5}\right]^{-}} & \delta 26.6\left(\mathrm{~d},{ }^{2} J_{\mathrm{F}-\mathrm{F}}=86.8\right) & \delta=-83.3 \text { (quintet, }{ }^{2} J_{\mathrm{F}-\mathrm{F}}=
\end{array}
$$


Die chemischen Verschiebungen der verbrückend wirkenden Fluoratome in $\mathbf{1 9}$ und $\left[\mathrm{HNEt}_{3}\right]^{+}\left[\mathrm{Cp}^{*} \mathrm{TaF}_{5}\right]^{-}$unterscheiden sich stark voneinander. Die Hochfeldverschiebung in 19 weist auf eine starke Bi-F-Wechselwirkung hin. Der Einfluß des Kations auf die endständigen Fluoratome ist erwartungsgemäß gering, was sich in einer dem $\mathrm{Cp}^{*} \mathrm{TaF}_{4}$ ähnlichen Verschiebung zeigt. Die ${ }^{2} J_{\mathrm{F}-\mathrm{F}}$-Kopplungskonstanten nehmen in beiden Verbindungen ähnliche Werte an. Sie unterscheiden sich aber von anderen $\left[\mathrm{RTaF}_{5}\right]^{-}$-Verbindungen, bei denen die Kopplungskonstanten Werte um die $25 \mathrm{~Hz}$ annehmen [98].

\subsubsection{Versuch zur Darstellung von $\mathrm{NsTaF}_{4}$}

Wie bereits erwähnt, führt die Fluorierung von $\mathrm{NsTaCl}_{4}$ mit $\mathrm{Me}_{3} \mathrm{SnF}$ zu dem Polymer $\left(\mathrm{Me}_{3} \mathrm{SnClMe}_{3} \mathrm{SnFTaF}_{5}\right)_{\mathrm{n}}$ [60]. Es erfolgte eine vollständige Substitution der Neosilylgruppe am Tantal-Metallzentrum. Aufgrund der unter 2.3.1. aufgestellten Vermutungen sollte $\mathrm{Ph}_{3} \mathrm{BiF}_{2} \mathrm{NsTaCl}_{4}$ unter Erhalt der Neosilylfunktion am Tantal fluorieren. Zu diesem Zweck wurde $\mathrm{NsTaCl}_{4}$ mit $\mathrm{Ph}_{3} \mathrm{BiF}_{2}$ in $\mathrm{CH}_{2} \mathrm{Cl}_{2}$ zur Reaktion gebracht. Nach zweistündigem Rühren bei Raumtemperatur wurden alle flüchtigen Bestandteile im Vakuum abgetrennt. Leider mißlang der Versuch, das Produkt mit Hilfe einer Sublimation bei 0.02 mbar und $60{ }^{\circ} \mathrm{C}$ abzutrennen. Eine Schwarzfärbung zeigte an, daß das Produkt zerstört wurde. Es ist wahrscheinlich, daß sich auch hier das Addukt $\left[\mathrm{Ph}_{3} \mathrm{BiCl}\right]^{+}\left[\mathrm{NsTaF}_{5}\right]^{-}$gebildet hat. Aus den Beispielen 2.3.7. und 2.3.8. läßt sich folgern, daß das Beiprodukt $\mathrm{Ph}_{3} \mathrm{Bi}(\mathrm{Cl}) \mathrm{F}$ zur Adduktbildung an Fluorokomplexe des Tantals neigt.

\subsubsection{Versuche zur Darstellung von Fluorokomplexen später Übergangsmetalle}

Das in Kap. 2.3.1. postulierte stärkere Bestreben der Bismut- und Bleifluoride gegenüber den Zinnfluoriden, andere Halogene gegen Fluor auszutauschen, soll im folgenden überprüft werden. $\mathrm{Zu}$ diesem Zweck wurden $\left(\mathrm{Cp}^{*} \mathrm{CrBr}_{2}\right)_{2}, \mathrm{Cp}^{*} \mathrm{MoCl}_{4}$ und $\left(\mathrm{Cp}^{*} \mathrm{RuCl}_{2}\right)_{2}$ Fluorierungen mit 13 und 14 unterworfen. Die Chrom- und Rutheniumkomplexe können auch nach mehrstündigem Refluxieren in Toluol unverändert zurückgewonnen werden. Bei dem Molybdänkomplex trat in beiden Fällen bereits bei Raumtemperatur eine Reaktion auf. Es 
entsteht ein blauer, in allen gängigen Lösungsmitteln unlöslicher Feststoff, der nicht weiter charakterisiert werden konnte.

\subsubsection{Abschließender Vergleich der Fluorierungsmittel $\mathrm{Me}_{3} \mathrm{SnF}(7), \mathrm{Ph}_{2} \mathrm{PbF}_{2}$ (13) und $\mathrm{Ph}_{3} \mathrm{BiF}_{2}(14)$}

Die Cl-F-Metathese mit 13 und 14 ist bei Metallkomplexen der 4. Gruppe erfolgreich. Wegen der schwierigeren Abtrennung der Beiprodukte $\mathrm{Ph}_{2} \mathrm{PbCl}_{2}$ und $\mathrm{Ph}_{3} \mathrm{BiCl}_{2}$ im Vergleich $\mathrm{zu} \mathrm{Me}_{3} \mathrm{SnCl}$ sind die Ausbeuten niedriger. Dieses Problem tritt bei der Darstellung von gut löslichen Fluorokomplexen mit 13 nicht auf, so daß $\mathrm{Cp}^{*} \mathrm{Cp}^{\prime} \mathrm{MF}_{2}(\mathrm{M}=\mathrm{Zr}$ (17), Hf (18)) in guten Ausbeuten isoliert werden können.

Fluorierungsversuche an Tantalkomplexen mit $\mathbf{1 4}$ haben gezeigt, daß auch hier ein Cl-F-Austausch stattfindet. Dabei wird eine Adduktbildung in Form von $\left[\mathrm{Ph}_{3} \mathrm{BiCl}\right]^{+}\left[\mathrm{Cp} * \mathrm{TaF}_{5}\right]^{-}$beobachtet, die eine Isolierung des reinen Fluorokomplexes verhindert.

Abschließend läßt sich sagen, daß keine Fluorokomplexe mit den neuen Fluorierungsmitteln synthetisiert werden konnten, die man nicht auch mit $\mathrm{Me}_{3} \mathrm{SnF}$ erhalten kann. $\mathrm{Ph}_{2} \mathrm{PbF}_{2}$ und $\mathrm{Ph}_{3} \mathrm{BiF}_{2}$ bieten gegenüber $\mathrm{Me}_{3} \mathrm{SnF}$ keinerlei Vorteile in Bezug auf Aufarbeitung, Ausbeute und Recycling. 


\subsubsection{Verwendung von Hydrogendifluoriden als Fluorierungsmittel}

$\mathrm{KHF}_{2}$ und $\mathrm{NH}_{4} \mathrm{HF}_{2}$ stehen als preiswerte $\mathrm{HF}_{2}{ }^{-}$-Quellen zur Verfügung. $\mathrm{KHF}_{2}$ wurde für Aufschlüsse von Silicaten [99], für die Darstellung von fluorierten Zuckern [100] und monofluorierten Phosphaten [101] verwendet. Um die Löslichkeit des Fluorierungsmittels in organischen Lösungsmitteln zu steigern, wird das anorganische Kation mittels quartären Ammonium- oder Phosphoniumhydrogensulfaten ausgetauscht [102].

$$
\begin{gathered}
\mathrm{Q}^{+} \mathrm{HSO}_{4}^{-}+\mathrm{M}^{+} \mathrm{HF}_{2}^{-} \longrightarrow \mathrm{Q}^{+} \mathrm{HF}_{2}^{-}+\mathrm{M}^{+} \mathrm{HSO}_{4}^{-} \\
\mathrm{M}^{+}=\mathrm{K}^{+}, \mathrm{NH}_{4}^{+} ; \mathrm{Q}^{+}=\mathrm{Et}_{4} \mathrm{~N}^{+}, n-\mathrm{Bu}_{4} \mathrm{~N}^{+}, \mathrm{Ph}_{4} \mathrm{P}^{+}
\end{gathered}
$$

Tetra- $n$-butylammoniumhydrogendifluorid (TBADF) ist eine ölige Substanz, die sich gut in aromatischen und halogenierten Kohlenwasserstoffen löst. Mit TBADF steht für die organische Chemie eine potente Fluoridquelle zur Verfügung. Aus organischen Chloriden, Bromiden und Tosylaten können durch eine nucleophile Substitutionen die entsprechenden Fluoride synthetisiert werden [103]. Eine andere Anwendung von TBADF in Kombination mit N-Halogensuccinimid (NBS, NCS) zeigt sich in der Halofluorierung von Olefinen [104].

Verbindungen des Siliciums können ebenfalls durch TBADF fluoriert werden. In diesen Reaktionen wird eine funktionelle Gruppe durch zwei Fluoratome ersetzt, so daß ein Anion entsteht. Diese Reaktion kann man gedanklich in zwei Teilreaktionen aufteilen, die einzeln schon realisiert wurden. Der erste Schritt beinhaltet die Substitution einer funktionellen Gruppe durch ein Fluoratom. Dieses kann mit Fluorierungsmitteln auf H-FBasis, z.B. Coll(HF $)_{2}$ und $\mathrm{Et}_{3} \mathrm{~N}(\mathrm{HF})_{3}$, erreicht werden. Danach wird mittels Koordination eines Fluoridions ein ionischer Fluorokomplex generiert. Mit Hilfe von TBADF lassen sich diese beiden Schritte in einem zusammenfassen.

$$
\begin{aligned}
& 2[\mathrm{M}] \mathrm{A}+\mathrm{Coll}(\mathrm{HF})_{2} \longrightarrow 2[\mathrm{M}] \mathrm{F}+2 \mathrm{HA}+\mathrm{Coll} \\
& {[\mathrm{M}] \mathrm{F}+n-\mathrm{Bu}_{4} \mathrm{NF} \longrightarrow\left[n-\mathrm{Bu}_{4} \mathrm{~N}\right]^{+}\left[[\mathrm{M}] \mathrm{F}_{2}\right]^{-}} \\
& {[\mathrm{M}] \mathrm{A}+\left[n-\mathrm{Bu}_{4} \mathrm{~N}\right]^{+}\left[\mathrm{HF}_{2}\right]^{-} \longrightarrow\left[n-\mathrm{Bu}_{4} \mathrm{~N}\right]+\left[[\mathrm{M}] \mathrm{F}_{2}\right]^{-}+\mathrm{HA}}
\end{aligned}
$$

$\mathrm{A}=$ Anion; Coll $=$ Collidin $; \mathrm{M}]=$ beliebiges Metallfragment 
Auf diesem Weg lassen sich trisubstituierte Silane $\left(\mathrm{R}_{3} \mathrm{SiH}, \mathrm{R}=\mathrm{Ph}\right)$ fluorieren [105]. Dabei kann man unter Freisetzung von Wasserstoff Difluorosilicate $\mathrm{R}_{3} \mathrm{SiF}_{2}{ }^{-}$erhalten. Derartige Systeme wurden zuvor aus Fluorosilanen und einer Fluoridquelle (Tetrabutylammoniumfluorid, TBAF) [106] oder Kronenether/Alkalimetallfluorid [107]) synthetisiert. Es sind auch entsprechende Zinnsysteme bekannt, die aus $\mathrm{R}_{3} \mathrm{SnF}$ und TBAF zugänglich sind [108]. Difluorosilicate und -stannate dienen als Fluoridquellen für nucleophile Substitutionen an Alkylhalogeniden und -tosylaten [109].

Ein einfacher Zugang zu Difluorodiorganometallaten der Elemente der 13. Gruppe wurde durch direkte Fluorierung der entsprechenden Metallorganyle mit TBADF eröffnet [110]. Diese Verbindungsklasse ist zwar seit 1955 bekannt [92, 111 - 113], es wurden aber erst wenige Verbindungen spektroskopisch und röntgenstrukturell charakterisiert [114 - 116]. Die Darstellung von $\left[\left(n-\mathrm{Bu}_{4} \mathrm{~N}\right)^{+}\right]_{2}\left[\left(\mathrm{RPO}_{4} \mathrm{AlF}_{2}\right)^{-}\right]_{2}$ ist ein weiteres Beispiel für eine Fluorierung durch TBADF [117]. Hierbei wurden Aluminiumphosphonate unter Methanabspaltung nach obigem Schema fluoriert.

$$
\begin{gathered}
\mathrm{RMMe}_{2}+\left[n-\mathrm{Bu}_{4} \mathrm{~N}\right]^{+}\left[\mathrm{HF}_{2}\right]^{-} \longrightarrow \begin{array}{l}
{\left[n-\mathrm{Bu}_{4} \mathrm{~N}\right]^{+}\left[\mathrm{RMeMF}_{2}\right]^{-}+\mathrm{MeH}} \\
\mathrm{M}=\mathrm{Al}, \mathrm{R}=\mathrm{Me}, \text { Tris; } \mathrm{M}=\mathrm{Ga}, \mathrm{R}=\mathrm{Me} ; \mathrm{M}=\mathrm{In}, \mathrm{R}=\mathrm{Me}
\end{array} \\
\left(\mathrm{RPO}_{3} \mathrm{AlMe}_{4}+4[n-\mathrm{BuN}]^{+}\left[\mathrm{HF}_{2}\right]^{-} \longrightarrow \begin{array}{l}
2\left[\left(n-\mathrm{Bu}_{4} \mathrm{~N}\right)^{+}\right]_{2}\left[\left(\mathrm{RPO}_{3} \mathrm{AlF}_{2}\right)^{-}\right]_{2} \\
+
\end{array}\right. \\
\mathrm{R}=\mathrm{Mh}, t-\mathrm{Bu}
\end{gathered}
$$

In der Literatur findet man nur ein Beispiel für eine Fluorierung eines Übergangsmetallkomplexes mit TBADF [117]. Das Titanalkoholat Ti(O $i$-Pr $)_{4}$ wurde mit TBADF zu einem zweikernigen Metallkomplex unter Abspaltung von einem Molekül Isopropanol umgesetzt und strukturell charakterisiert.

$$
\begin{aligned}
& 2 \mathrm{Ti}(\mathrm{O} i-\mathrm{Pr})_{4}+\left[n-\mathrm{Bu}_{4} \mathrm{~N}\right]^{+}\left[\mathrm{HF}_{2}\right]^{-} \longrightarrow \\
& {\left[n-\mathrm{Bu}{ }_{4} \mathrm{~N}\right]^{+}\left[(\mathrm{O} i-\mathrm{Pr})_{3} \operatorname{Ti}(\mu \mathrm{F})_{2}(\mu \mathrm{O} i-\mathrm{Pr}) \operatorname{Ti}(\mathrm{O} i \operatorname{Pr})_{3}\right]^{-}+i-\operatorname{PrOH} }
\end{aligned}
$$

Der Unterschied $\mathrm{zu}$ den zuvor gezeigten Reaktionen liegt darin, daß hier ein Äquivalent Fluorierungsmittel mit zwei Äquivalenten Alkoholat reagiert. Dabei ist die 
Ausbildung des Zwischenprodukts $\left[n-\mathrm{Bu}_{4} \mathrm{~N}\right]^{+}\left[(\mathrm{O} i-\mathrm{Pr})_{3} \mathrm{TiF}_{2}\right]^{-}$denkbar, das dann unter Erhöhung der Koordinationszahl von 5 auf 6 mit einem weiteren Titanalkoholat reagiert.

\subsubsection{Fluorierungen von Metallalkoxiden}

\subsubsection{Darstellung von $\mu_{3}$-Fluoro- $\mu_{3}$-oxo-tris( $\mu$-isopropoxi)-hexakis(isopropoxi)-tri- $\operatorname{titan}(I V)(20)$}

Die Umsetzung von $\mathrm{Ti}(\mathrm{O} i \mathrm{Pr})_{4}$ mit TBADF ergab einen zweikernigen Titankomplex (Kap. 2.4.1.). Aus diesem Grund wurden $\mathrm{KHF}_{2}$ und $\mathrm{Ti}(\mathrm{O} i \mathrm{Pr})_{4}$ in einem molaren Verhältnis von $1: 2$ zur Reaktion gebracht. Aufgrund der geringen Löslichkeit von $\mathrm{KHF}_{2}$ in allen gängigen Lösungsmitteln konnte bei Raumtemperatur auch nach mehrtägigem Rühren keine Reaktion beobachtet werden. Nach sechsstündigem Erhitzen unter Rückfluß in Toluol hatte sich die Korngröße des Fluorierungsmittels merklich verkleinert. Eine Elementaranalyse des Rückstands ergab, daß eine Mischung von $\mathrm{KHF}_{2}$ und $\mathrm{KF}$ vorlag. Das Verhältnis der beiden Komponenten zeigt an, daß genau drei Äquivalente $\mathrm{Ti}(\mathrm{O} i \text {-Pr })_{4}$ mit einem Äquivalent $\mathrm{KHF}_{2}$ reagiert haben. Das Fluorierungsmittel $\mathrm{KHF}_{2}$ zeigt in diesem Fall ein mit HF vergleichbares Reaktionsverhalten. Es erfolgte also eine Substitution eines Alkoholat- durch einen Fluoridliganden. Der Einbau eines dreifachverbrückenden Sauerstoffatoms läßt sich durch Bildung eines Ethermoleküls aus zwei Alkoholatresten erklären.

$$
\mathrm{KHF}_{2}+3 \mathrm{Ti}(\mathrm{O} i-\mathrm{Pr})_{4}
$$

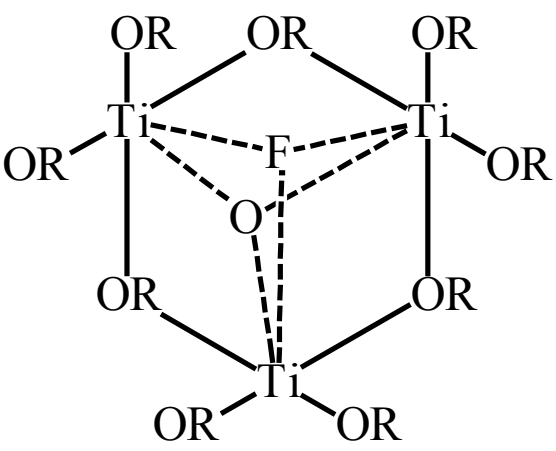

20

Verbindung 20 wurde mittels Elementaranalysen, NMR- und IR-Spektroskopie sowie Massenspektrometrie charakterisiert. Im ${ }^{19}$ F-NMR-Spektrum findet man bei Raumtemperatur 
drei Signale bei $\delta-130.2,-122.4$ und $110.4 \mathrm{ppm}$. Durch die Formulierung eines Gleichgewichts in Lösung (Schema 1) kann man dieses Phänomen erklären. Aufgrund der chemischen Verschiebung lassen sich die beiden Signale bei hohem Feld verbrückenden (20, 20a) und das bei tiefem Feld endständigen Fluoratomen (20b) zuordnen [5].

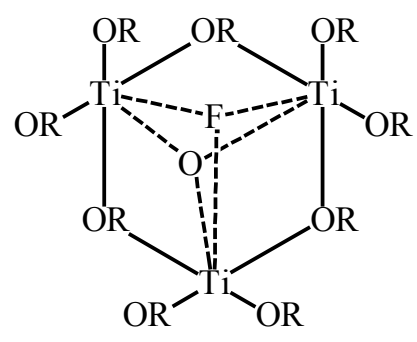

20

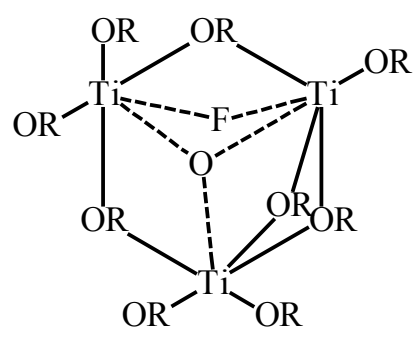

20a

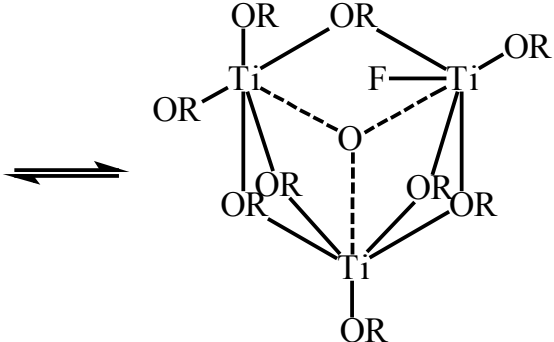

20b

Schema 1: Isomere von Verbindung 20 in Lösung

Um diese Behauptung zu untermauern, wurden ${ }^{19} \mathrm{~F}-\mathrm{NMR}$-Spektren bei verschiedenen Temperaturen aufgenommen. Wie zu erwarten war, wird das Integral für das Signal von 20 mit fallender Temperatur stärker und für 20a und 20b schwächer. Ein Vergleich der Intensitäten der drei Signale bei verschiedenen Temperaturen ist aus Tabelle $13 \mathrm{zu}$ entnehmen.

Tabelle 13: ${ }^{19}$ F-NMR-Spektraldaten der Verbindungen 20, 20a und 20b bei verschiedenen Temperaturen und 1/T $\left[1 / \mathrm{K} \times 10^{-3}\right]$ und $\ln \mathrm{K}_{(\mathrm{T})}$ (Angaben der Intensitäten in \%)

$\begin{array}{rccccc}\mathrm{T}\left[{ }^{\circ} \mathrm{C}\right] & \delta-130.15 \mathrm{ppm} & \delta-122.39 \mathrm{ppm} & \delta 110.40 \mathrm{ppm} & 1 / \mathrm{T}\left[1 / \mathrm{K} \times 10^{-3}\right] & \ln \mathrm{K}_{(\mathrm{T})} \\ 30 & 56 & 23 & 21 & (3.3003) & (-0.8916) \\ 25 & 75 & 13 & 12 & 3.3557 & -1.7719 \\ 0 & 88 & 6 & 6 & 3.6630 & -2.6882 \\ -25 & 94 & 3 & 3 & 4.0323 & -3.4420 \\ -50 & 98 & 1 & 1 & 4.4843 & -4.6052\end{array}$


Die Gleichgewichtskonstante $\mathrm{K}_{(\mathrm{T})}$ wird aus dem Verhältnis der Integrale der Signale bei $\delta-122.39$ und -130.15 ppm berechnet. Eine Auftragung von $\ln \mathrm{K}_{(\mathrm{T})}$ gegen $1 / \mathrm{T}$ ergibt eine Gerade (Diagramm 1), wenn man annimmt, daß die Reaktionsenthalpie in dem betrachteten Temperaturintervall konstant ist. Das Wertepaar bei einer Temperatur von $30^{\circ} \mathrm{C}$ wurde bei der Auftragung nicht berücksichtigt, weil bei dieser Messung die Basislinie im NMR stark schwankte und folglich das Integral mit einem großen Fehler behaftet ist. Aus der Steigung läßt sich die Reaktionsenthalpie und aus dem Y-Achsenabschnitt die Reaktionsentropie berechnen. Es gilt [118]:

$$
\begin{array}{ll}
\Delta \mathrm{G}=\Delta \mathrm{H}-\mathrm{T} \times \Delta \mathrm{S} & {[1]} \\
\Delta \mathrm{G}=-\mathrm{R} \times \mathrm{T} \times \ln \mathrm{K} & {[2]} \\
\ln \mathrm{K}=(\Delta \mathrm{S} / \mathrm{R})-1 / \mathrm{T} \times(\Delta \mathrm{H} / \mathrm{R}) & {[3]}
\end{array}
$$

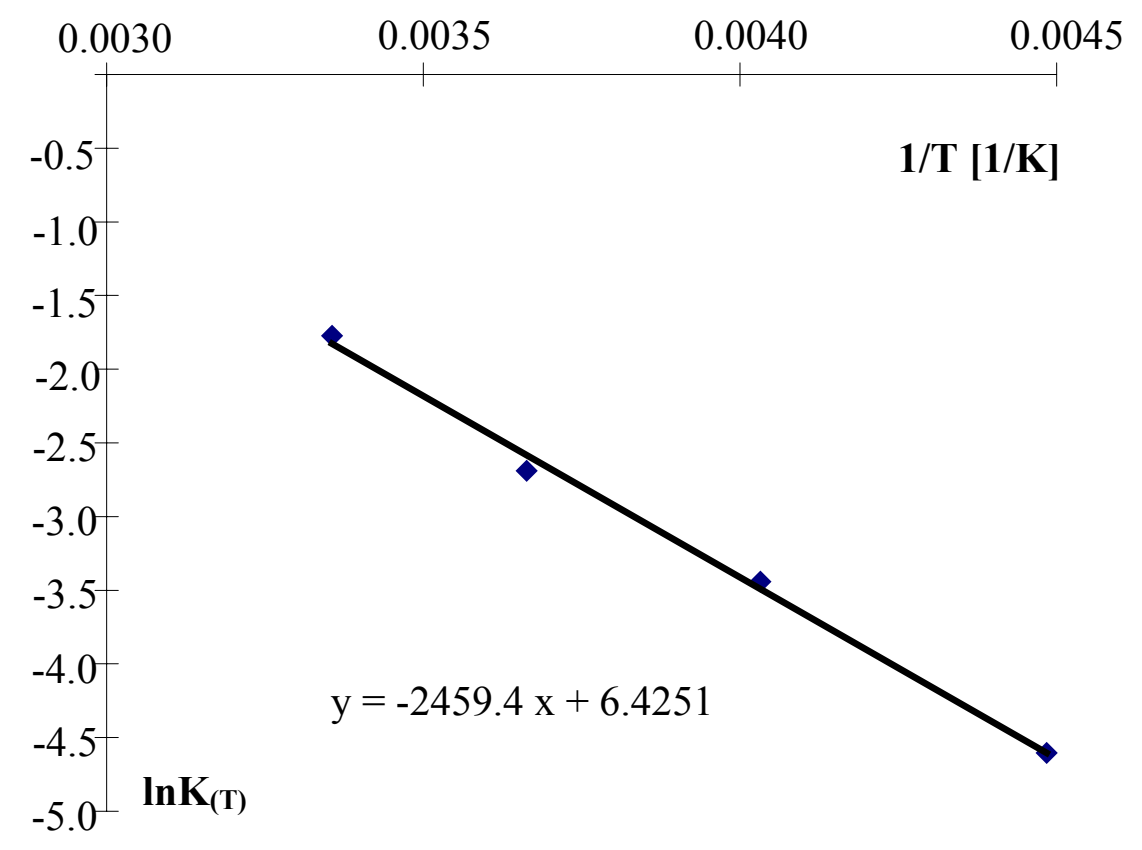

Diagramm 1: Auftragung von $\ln \mathrm{K}_{(\mathrm{T})}$ gegen $1 / \mathrm{T}$

Aus der Auftragung wurden $\Delta \mathrm{H}^{\circ}(20.4 \pm 0.9) \mathrm{kJ} / \mathrm{mol}$ und $\Delta \mathrm{S}^{\circ}(53.2 \pm 3.3) \mathrm{J} / \mathrm{mol} \mathrm{x} \mathrm{K}$ $\left(\mathrm{R}^{2}=0.996\right)$ bestimmt. Messungen von Bradley et al. ergaben für eine $\mathrm{Zr}-\mathrm{OR} \rightarrow \mathrm{Zr}$ Bindung eine Energie von $37 \mathrm{~kJ} / \mathrm{mol}$. In demselben Artikel wird gezeigt, daß ein entsprechendes Titansystem eine vergleichbare Bindungsenergie aufweist [119]. Die Enthalpie der Reaktion 
$20 \rightarrow 20 a$ ist geringer, weil hier zusätzlich ein $\mu_{3}$-F-Atom $\mathrm{zu}$ einem $\mu$ F-Atom umlagert. Dieser Transformation läßt sich rechnerisch eine Energie von $-17 \mathrm{~kJ} / \mathrm{mol}$ zuordnen.

Es ist auffällig, daß die Isomere 20a und 20b über den ganzen Temperaturbereich in der gleichen Konzentration vorliegen. Entweder ist $\Delta \mathrm{H}^{\circ}$ des Gleichgewichts der Isomere gleich Null, oder sie liegen dimer vor. Schema 2 zeigt einen Strukturvorschlag für ein Dimer aus 20a und 20b.

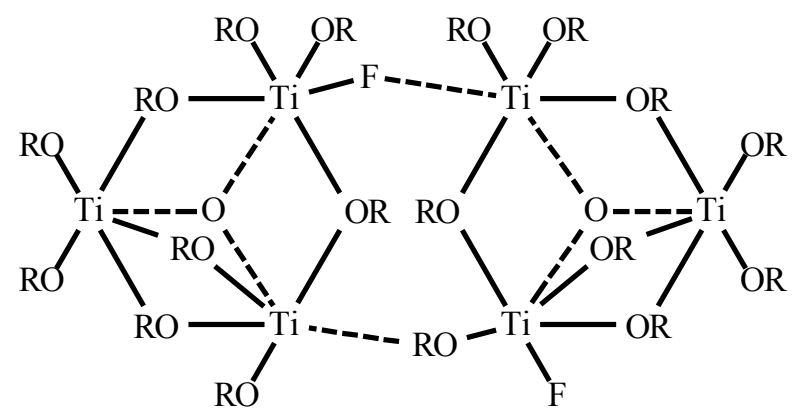

Schema 2: Strukturvorschlag für das Dimer 20ab in Lösung

Im Isomer 20a gibt es für das $\mu \mathrm{F}$-Atom zwei Möglichkeiten für einen Bindungsbruch, so daß es von zwei unterschiedlichen Titanatomen aus die beiden Isomere verbrücken kann. Im Koordinationspartner stehen dem $\mu$ F-Atom drei unterschiedliche Titanatome zur Verfügung. Nach Koordination mit 20b kann das endständige Fluoratom an eines dieser drei Titanatome gebunden sein. Außerdem gibt es drei Möglichkeiten, die beiden von zwei Alkoxigruppen verbrückten Titanatome im ehemaligen Isomer 20b anzuordnen. Es können folglich $18(2 \times 3 \times 3)$ Isomere von 20 ab formuliert werden.

Von Verbindung 20 konnten aus $\mathrm{Et}_{2} \mathrm{O}$ über Nacht bei $-30{ }^{\circ} \mathrm{C}$ Einkristalle gewonnen werden, die röntgenstrukturell charakterisiert werden konnten. Es wurde die monokline Raumgruppe $P 2_{1} / n$ gefunden. Die Struktur von Verbindung 20 im Festkörper zeigt, daß ein sechsgliedriges Ringsystem alternierend bestehend aus Titan und Alkoholat vorliegt. Im Zentrum dieses Systems befinden sich je ein Sauerstoff- und Fluoratom. Das gleiche Grundgerüst findet man bei $\mu_{3}$-Chloro- $\mu_{3}$-oxo-tris( $\mu$-neopentoxi)-hexakis(neopentoxi)-trititan(IV) [120], allerdings nimmt hier ein Chloratom die Position des Fluoratoms ein. Es existieren viele Titanverbindungen, die dreifach verbrückende Sauerstoffatome enthalten [120, 121, 122], $\mu_{3}$-F-Atome enthaltende Titanverbindungen sind bislang unbekannt. Der 
durchschnittliche $\mu_{3}-\mathrm{O}-\mathrm{Ti}$ Bindungsabstand in 20 beträgt $205.4 \mathrm{pm}$ und stimmt mit den Literaturwerten überein. Der mittlere $\mu_{3}-\mathrm{F}-\mathrm{Ti}$-Abstand ist mit $214.4 \mathrm{pm}$ erwartungsgemäß länger im Vergleich zum Metall-Sauerstoff-Bindungsabstand. Der Komplex $\left[\left(\mathrm{Cp}^{*} \mathrm{TiF}_{3}\right)_{4} \mathrm{CaF}_{2}\right]$ [67] besitzt ein $\mu_{4}$-F-Atom mit einer Bindung zu einem Calcium- und drei Titanatomen. Der Ti-F-Bindungsabstand beträgt in diesem Fall 217.5 pm und liegt damit in der gleichen Größenordnung wie bei Verbindung 20. Abbildung 10 zeigt die Struktur von 20 im Festkörper und charakteristische Bindungslängen und -winkel sind in Tabelle 14 aufgeführt.

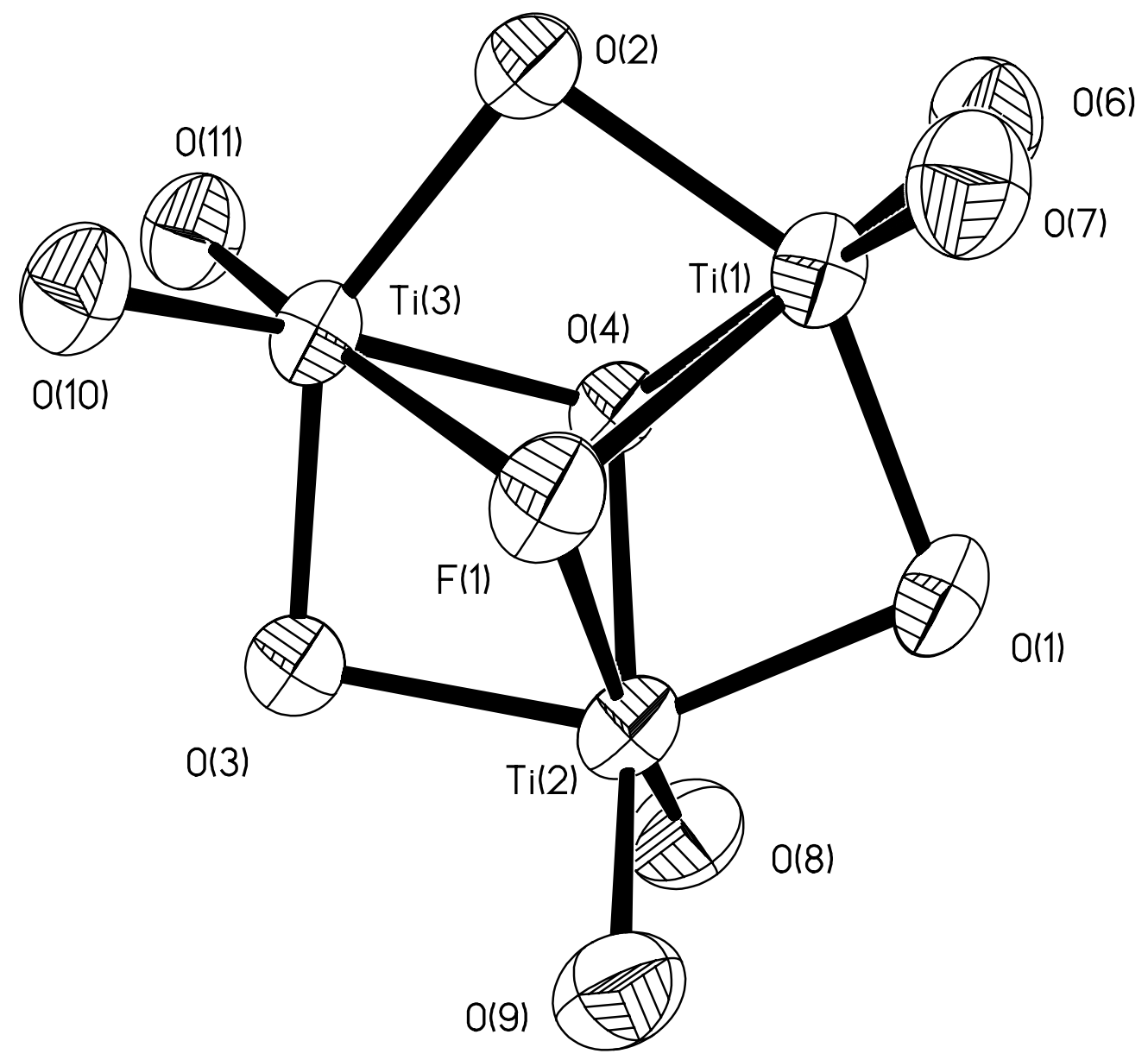

Abbildung 10: Struktur von Verbindung 20 im Festkörper 
Tabelle 14: Charakteristische Bindungslängen (pm) und -winkel $\left(^{\circ}\right)$ von 20

$\begin{array}{llll}\operatorname{Ti}(1)-\mathrm{F}(1) & 216.3(3) & \mathrm{F}(1)-\mathrm{Ti}(1)-\mathrm{O}(4) & 70.26(13) \\ \operatorname{Ti}(2)-\mathrm{F}(1) & 213.3(3) & \mathrm{F}(1)-\mathrm{Ti}(2)-\mathrm{O}(4) & 70.94(13) \\ \operatorname{Ti}(3)-\mathrm{F}(1) & 213.5(3) & \mathrm{F}(1)-\mathrm{Ti}(3)-\mathrm{O}(4) & 70.69(12) \\ \operatorname{Ti}(1)-\mathrm{O}(4) & 205.2(3) & & \\ \operatorname{Ti}(2)-\mathrm{O}(4) & 204.9(3) & & \\ \operatorname{Ti}(3)-\mathrm{O}(4) & 206.0(3) & & \end{array}$

\subsubsection{Reaktion von $\mathrm{Zr}(\mathrm{O} t-\mathrm{Bu})_{4}$ mit $\mathrm{KHF}_{2}$}

Es war nun von Interesse $\mathrm{zu}$ untersuchen, ob sich Zirconiumalkoholate in den Fluorierungsreaktionen mit $\left[n-\mathrm{Bu}_{4} \mathrm{~N}\right]^{+}\left[\mathrm{HF}_{2}\right]^{-}$und $\mathrm{KHF}_{2}$ ähnlich wie das Titanalkoholat $\mathrm{Ti}(\mathrm{O} i \text {-Pr })_{4}$ verhalten. Als Edukt wurde $\mathrm{Zr}(\mathrm{O} t-\mathrm{Bu})_{4}$ ausgewählt, weil es aufgrund des großen Platzbedarfs des $t$-Butanolats ausschließlich monomer vorliegt. Das Vorliegen von nur einem Isomer in Lösung verspricht eine bessere Kontrollierbarkeit der Reaktion und eine größere Chance, auch nur ein Produkt zu erhalten. $\mathrm{Zr}(\mathrm{O} t-\mathrm{Bu})_{4}$ wurde mit $\mathrm{KHF}_{2}$ in einem molaren Verhältnis von $1: 1$ in Toluol unter Rückfluß erhitzt. Nach Kristallisation aus Ether konnte ein farbloses Produkt isoliert werden. Aufschluß über das Kation lieferte ein FAB-MS, das bei m/z 947 einen Peak zeigt, dessen Isotopenverteilung exakt für die berechnete des Kations von 21 übereinstimmt (Schema 3). Das ${ }^{1} \mathrm{H}-\mathrm{NMR}$ zeigt drei verschiedene Signale für Protonen der $t$-Bu-Gruppen bei $\delta 1.30,1.40$ und $1.61 \mathrm{ppm}$ mit nahezu gleicher Intensität. Die beiden im hohen Feld liegenden Signale stammen vermutlich von den Protonen der sechs endständigen Alkoholatreste. Da sie axiale und äquatoriale Positionen einnehmen können, erhält man zwei Signale. Das Signal bei $\delta 1.60 \mathrm{ppm}$ stammt von Protonen der Alkoxigruppen, die den sechsgliedrigen Ring aufbauen. Ein weiteres Signal bei $\delta 1.58 \mathrm{ppm}$ mit nur etwa 1/3 der Intensität der anderen gibt einen Hinweis auf das Anion $\mathrm{O} t-\mathrm{Bu}^{-}$. Diese Annahme wird auch durch das IR-Spektrum gestützt, das keinen Hinweis auf $\mathrm{OH}^{-}$liefert. Im ${ }^{19} \mathrm{~F}-\mathrm{NMR}$ findet man nur sehr schwache Signale, so daß davon auszugehen ist, daß das Produkt fluorfrei ist, womit Fluorid als Anion ebenfalls ausscheidet. 


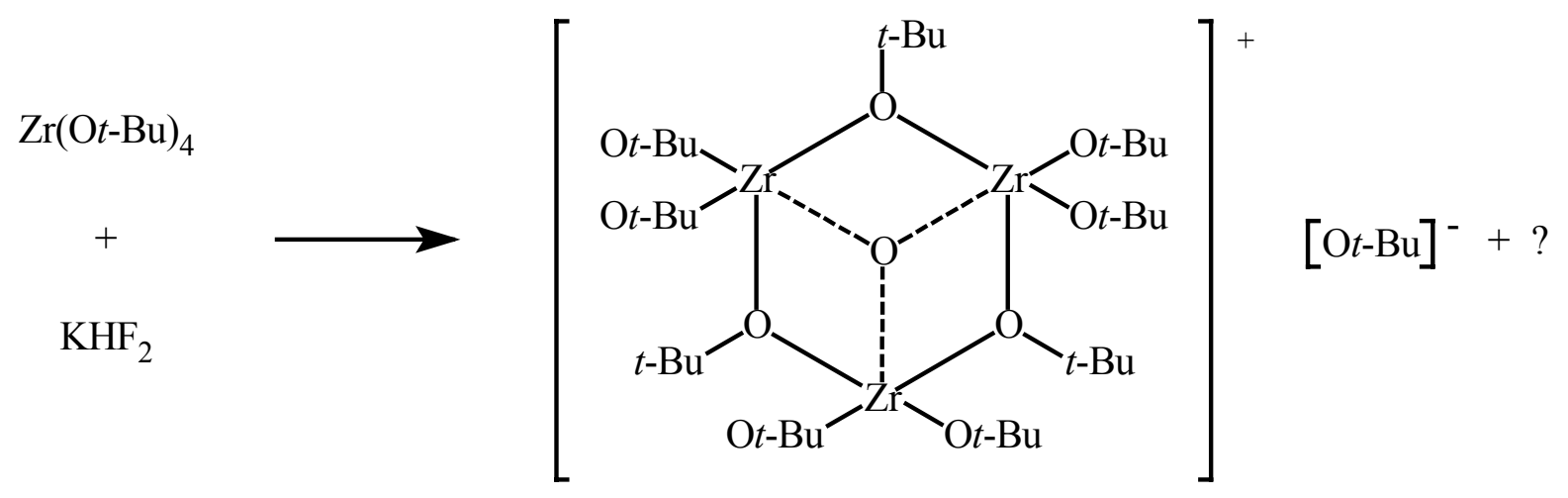

21

Schema 3: Wahrscheinliches Reaktionsprodukt von $\mathrm{KHF}_{2}$ und $\mathrm{Zr}(\mathrm{O} t-\mathrm{Bu})_{4}$

\subsubsection{Darstellung von $\left[n-\mathrm{Bu}_{4} \mathrm{~N}\right]^{+}\left[(\mathrm{O} t-\mathrm{Bu})_{2} \mathrm{ZrF}_{3}\right]^{-}(22)$}

Um eine Etherabspaltung wie unter 2.4.2.3. zu verhindern, wurde TBADF als Fluorierungsmittel gewählt. Aufgrund der guten Löslichkeit von $\left[n-\mathrm{Bu}_{4} \mathrm{~N}\right]^{+}\left[\mathrm{HF}_{2}\right]^{-}$in organischen Lösungsmitteln muß hier im Gegensatz zu KHF $\mathrm{KH}_{2}$ nicht erhitzt werden. $\mathrm{Zr}(\mathrm{O} t \text { - } \mathrm{Bu})_{4}$ wurde im molaren Verhältnis 1:1 mit TBADF zur Reaktion gebracht. Nach Umkristallisation aus $\mathrm{CH}_{2} \mathrm{Cl}_{2}$ wurde ein farbloser Feststoff isoliert.

$$
\mathrm{Zr}(\mathrm{O} t-\mathrm{Bu})_{4}+\left[n-\mathrm{Bu}_{4} \mathrm{~N}\right]^{+}\left[\mathrm{HF}_{2}\right]^{-} \quad \underset{-t-\mathrm{BuOH}}{\stackrel{\mathrm{CH}_{2} \mathrm{Cl}_{2}}{\longrightarrow}} \quad\left[n-\mathrm{Bu}_{4} \mathrm{~N}\right]^{+}\left[(t-\mathrm{BuO})_{2} \mathrm{ZrF}_{3}\right]^{-}+?
$$

Das ${ }^{1} \mathrm{H}-\mathrm{NMR}-$ Spektrum des Kristallisats zeigt ein Signal bei $\delta 1.26 \mathrm{ppm}$, das von den Protonen einer $t$-Bu-Gruppe stammt. Die Integrale lassen auf ein Verhältnis von $\left[n-\mathrm{Bu}_{4} \mathrm{~N}\right]^{+} \mathrm{zu}$ Alkoholat von $1: 2$ schließen. Die chemische Verschiebung des Signals im ${ }^{19}$ F-NMR bei $\delta$ -11.8 ppm weist auf endständige Fluoratome hin. Im FAB-MS findet man einen Peak für das Kation bei m/z 242, welcher sich $\left[\mathrm{Bu}_{4} \mathrm{~N}\right]^{+}$zuordnen läßt. Das Anion liefert ein Signal bei m/z 446 welches auf $\left[(t-\mathrm{BuO}){ }_{2} \mathrm{ZrF}_{3}\right]^{-} \times \mathrm{NBA}(\mathrm{NBA}=3$-Nitrobenzylalkohol) schließen läßt, was durch die Isotopenverteilung bestätigt wird. Der NBA stammt aus der Matrix bei der FABMessung. Im IR-Schwingungsspektrum findet man eine intensive Bande bei $486 \mathrm{~cm}^{-1}$, die einen weiteren Hinweis auf eine Zr-F-Bindung liefert [123]. Die Elementaranalyse bestätigt die Zusammensetzung von 22. Es fällt auf, daß bei der Reaktion von $\mathrm{Zr}(\mathrm{O} t-\mathrm{Bu})_{4}$ mit TBADF 
zwei Alkoxigruppen gegen drei Fluoridionen ersetzt wurden. Dieses Substitutionsmuster war bislang noch nicht bekannt. Möglicherweise steuert die Größe des Alkoxidrests die Produktbildung. Um diese Frage $\mathrm{zu}$ beantworten, sind weitere Fluorierungsversuche notwendig.

\subsubsection{Darstellung von $\operatorname{Bis}\left(\mu_{3}\right.$-oxo)-hexakis( $\mu$-ethoxi)-octakis( $(\mu$-oxo)- tetradekakis(ethoxi)-octatantal(V) (23)}

Weitere interessante Edukte für Fluorierungen mit TBADF oder $\mathrm{KHF}_{2}$ sind die Metallalkoxide der 5. Gruppe. Bei der Umsetzung von $\mathrm{VO}(\mathrm{O} i-\mathrm{Pr})_{3}$ mit $[n-\mathrm{BuN}]^{+}\left[\mathrm{HF}_{2}\right]^{-}$ konnte bei Raumtemperatur und in siedendem $\mathrm{CH}_{2} \mathrm{Cl}_{2}$ keine Reaktion beobachtet werden. Als weiteres Metallalkoxid dieser Gruppe wurde $\mathrm{Ta}(\mathrm{OEt})_{5}$ der Fluorierung unterworfen. Es wurde ein Molverhältnis Alkoholat zum Fluorierungsmittel von 2:1 gewählt, weil die entsprechende Reaktion mit Ti(Oi-Pr $)_{4}$ der gleichen Stöchometrie folgt. Bei der Umsetzung mit $\mathrm{KHF}_{2}$ konnte selbst in siedendem Toluol keine Reaktion beobachtet werden. Bei der Umsetzung mit TBADF hingegen war bereits bei Raumtemperatur eine Reaktion zu beobachten. Das ${ }^{1} \mathrm{H}-\mathrm{NMR}-S p e k t r u m$ des Rohprodukts zeigt Signale für $\left[n-\mathrm{Bu}_{4} \mathrm{~N}\right]^{+}-$und $\mathrm{OEt}^{-}-$ Gruppen. Aus dem Verhältnis ihrer Integrale läßt sich ableiten, daß von jedem TantalMetallzentrum im Mittel 1.25 Alkoxigruppen abgespalten wurden. Im ${ }^{19}$ F-NMR-Spektrum findet man zwei Signale bei $\delta-42.8$ und $-33.7 \mathrm{ppm}$. Die chemischen Verschiebungen weisen auf verbrückende Fluoratome hin. Aus Toluol konnten bei $-30{ }^{\circ} \mathrm{C}$ für eine Strukturanalyse geeignete Kristalle eines Nebenprodukts gewonnen werden. Abbildung 11 zeigt die Struktur von 23 im Festkörper und in Tabelle 15 sind charakteristische Bindungslängen aufgeführt. 


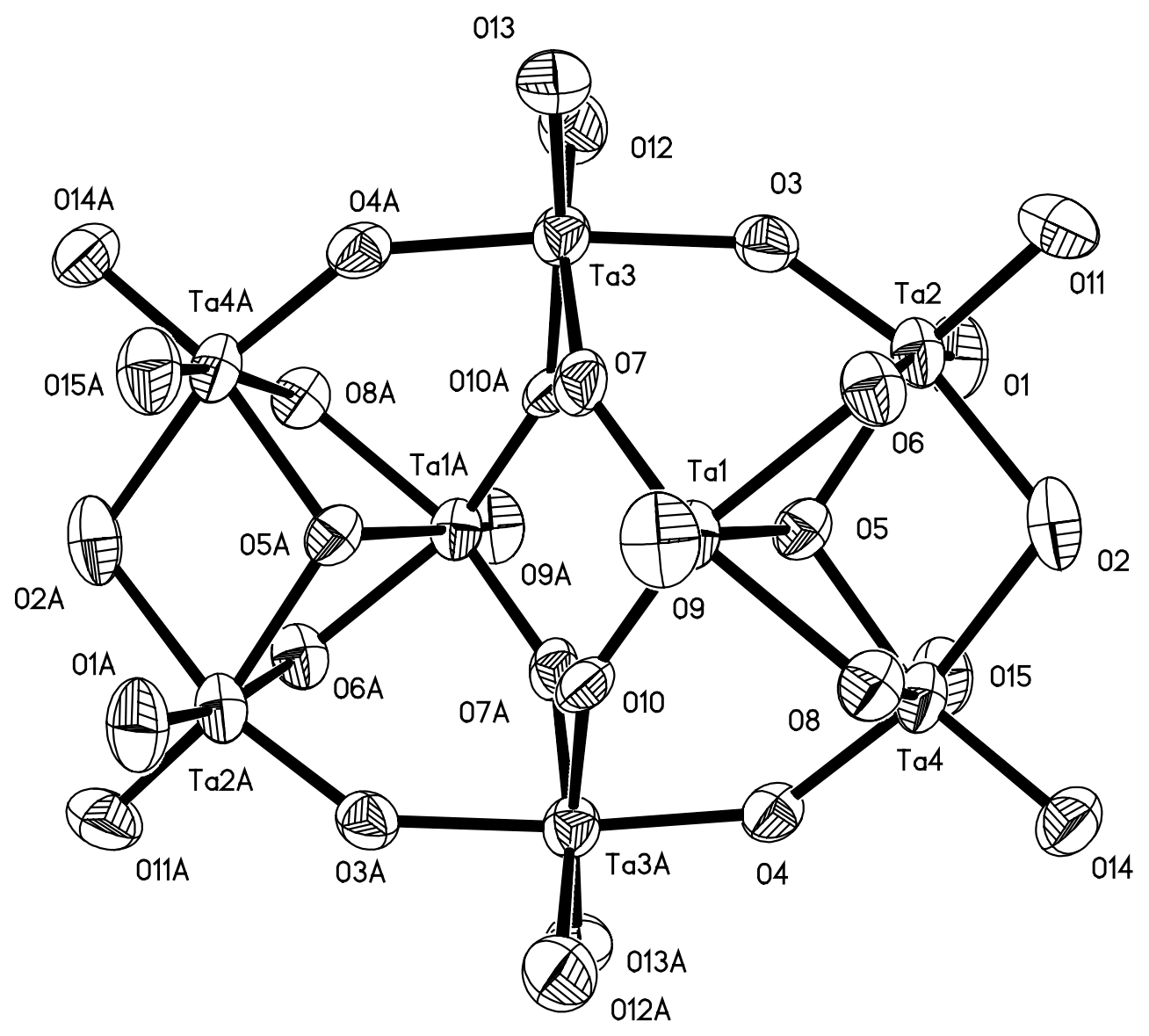

Abbildung 11: Struktur von 23 im Festkörper (ohne C- und H-Atome)

Tabelle 15: Charakteristische Bindungslängen (pm) von 23

$\mathrm{Ta}(1)-\mathrm{O}(5) 2.087(9)$ $\mathrm{Ta}(4)-\mathrm{O}(2) 2.150(11)$

$\mathrm{Ta}(1)-\mathrm{O}(9) 1.865(9)$ Ta-(2)-O(5) 2.059(10)

$\mathrm{Ta}(4)-\mathrm{O}(5) 2.023(9)$

$\mathrm{Ta}(2)-\mathrm{O}(3) 1.846(10)$

$\mathrm{Ta}(3)-\mathrm{O}(3) 2.018(10)$

$\mathrm{Ta}(2)-\mathrm{O}(2) 2.128(12)$

Die Einkristallröntgenstrukturanalyse von 23 liefert ein überraschendes Ergebnis. Bei dem Kristallisat handelt es sich um ein Nebenprodukt, das keine Fluoratome enthält. Unter Abspaltung von Diethylether ist ein großer Cluster bestehend aus 8 Tantal-Metallzentren, 10 Sauerstoffatomen und 20 Alkoxid-Gruppen entstanden. Dieses punktsymmetrische Molekül 
wird aus zwei sechsgliedrigen Ringen, in deren Zentrum sich ein dreifachgebundenes Sauerstoffatom befindet, vier weiteren sechsgliedrigen Ringen und einem achtgliedrigen Ring im Zentrum des Moleküls aufgebaut. Eine vergleichbare Struktur ist bekannt, bei der die Tantal- durch Niobatome ersetzt sind [124]. Sie wurde aus $\mathrm{NbO}(\mathrm{OEt})_{3}$ durch Freisetzung von $\mathrm{Et}_{2} \mathrm{O}$ nach längerer Lagerung bei Raumtemperatur erhalten.

\subsubsection{Darstellung von $\left[\left(n-\mathrm{Bu}_{4} \mathrm{~N}\right)^{+}\right]_{2}\left[\mathrm{SiF}_{6}\right]^{2-}(24)$}

Die Fluorierung von Siliciumalkoxiden mit TBADF ist bislang nicht bekannt. Tetraethoxisilan $\left(\mathrm{Si}(\mathrm{OEt})_{4}\right)$ wurde mit TBADF in einem molaren Verhältnis von $1: 1$ bei Raumtemperatur in $\mathrm{CH}_{2} \mathrm{Cl}_{2}$ zur Reaktion gebracht. Nach Umkristallisation aus $\mathrm{CH}_{2} \mathrm{Cl}_{2}$ entstand das kristalline Reaktionsprodukt 24.

$$
\mathrm{Si}(\mathrm{OEt})_{4}+\left[n-\mathrm{Bu}_{4} \mathrm{~N}\right]^{+}\left[\mathrm{HF}_{2}\right]^{-} \quad \frac{\mathrm{CH}_{2} \mathrm{Cl}_{2}}{-\mathrm{OEt}} \quad\left[\left(n-\mathrm{Bu}_{4} \mathrm{~N}\right)^{+}\right]_{2}\left[\mathrm{SiF}_{6}\right]^{2-}+?
$$

Bei dieser Umsetzung war die Bildung von $\left[\mathrm{Bu}_{4} \mathrm{~N}\right]^{+}\left[(\mathrm{EtO})_{3} \mathrm{SiF}_{2}\right]^{-} \mathrm{zu}$ erwarten. Betrachtet man das ${ }^{1}$ H-NMR-Spektrum, so fällt auf, daß Signale der Protonen aus dem Ethanolatrest vollständig fehlen. Im ${ }^{29}$ Si-NMR-Spektrum findet man ein Heptett, woraus man folgern kann, daß sechs Fluoratome am Silicium gebunden sind, was auf das $\left[\mathrm{SiF}_{6}\right]^{2-}$-Ion schließen läßt. Da man im ${ }^{19}$ F-NMR-Spektrum nur ein Signal findet, müssen alle Fluoratome chemisch äquivalent sein. Das FAB-MS zeigt für das Kation einen Peak, der sich $\left[n-\mathrm{Bu}_{4} \mathrm{~N}\right]^{+}$ zuordnen läßt. Das Anion liefert bei m/z 276 einen Peak, der auf $\left[\mathrm{SiF}_{5}\right]^{-} \times \mathrm{NBA}$ schließen läßt. Eine Einkristallröntgenstrukturanalyse war nicht erfolgreich. 


\subsubsection{Alternative Darstellung von $\left[\mathrm{Bu}_{4} \mathrm{~N}\right]^{+}\left[\mathrm{Me}_{2} \mathrm{AlF}_{2}\right]^{-}$(25)}

Die Verbindung 25 wurde bereits aus TBADF und $\mathrm{AlMe}_{3}$ hergestellt [110]. In diesem Zusammenhang erschien es von Interesse, wie sich $\left(t-\mathrm{BuOAlMe}_{2}\right)_{2}$ in einer Fluorierungsreaktion mit $\left[n-\mathrm{Bu}_{4} \mathrm{~N}\right]^{+}\left[\mathrm{HF}_{2}\right]^{-}$verhalten würde. Es war die Darstellung von $\left[n-\mathrm{Bu}_{4} \mathrm{~N}\right]^{+}\left[\mathrm{MeAl}(\mathrm{O} t-\mathrm{Bu}) \mathrm{F}_{2}\right]^{-}$geplant, weil es einfacher erscheint, eine Al-C-als eine Al-OBindung zu spalten. Stattdessen entsteht unter $t$-Butanol Abspaltung $\left[n-\mathrm{Bu}_{4} \mathrm{~N}\right]^{+}\left[\mathrm{Me}_{2} \mathrm{AlF}_{2}\right]^{-}$. Das ${ }^{1}$ H-NMR-Spektrum weist keine Signale von Protonen der $t$-Butylgruppen auf. Ein Vergleich der NMR-Spektren mit den Literaturwerten bestätigt die Bildung von 25. Die hier beschriebene Darstellungsmethode stellt gegenüber der literaturbekannten keine Verbesserung dar.

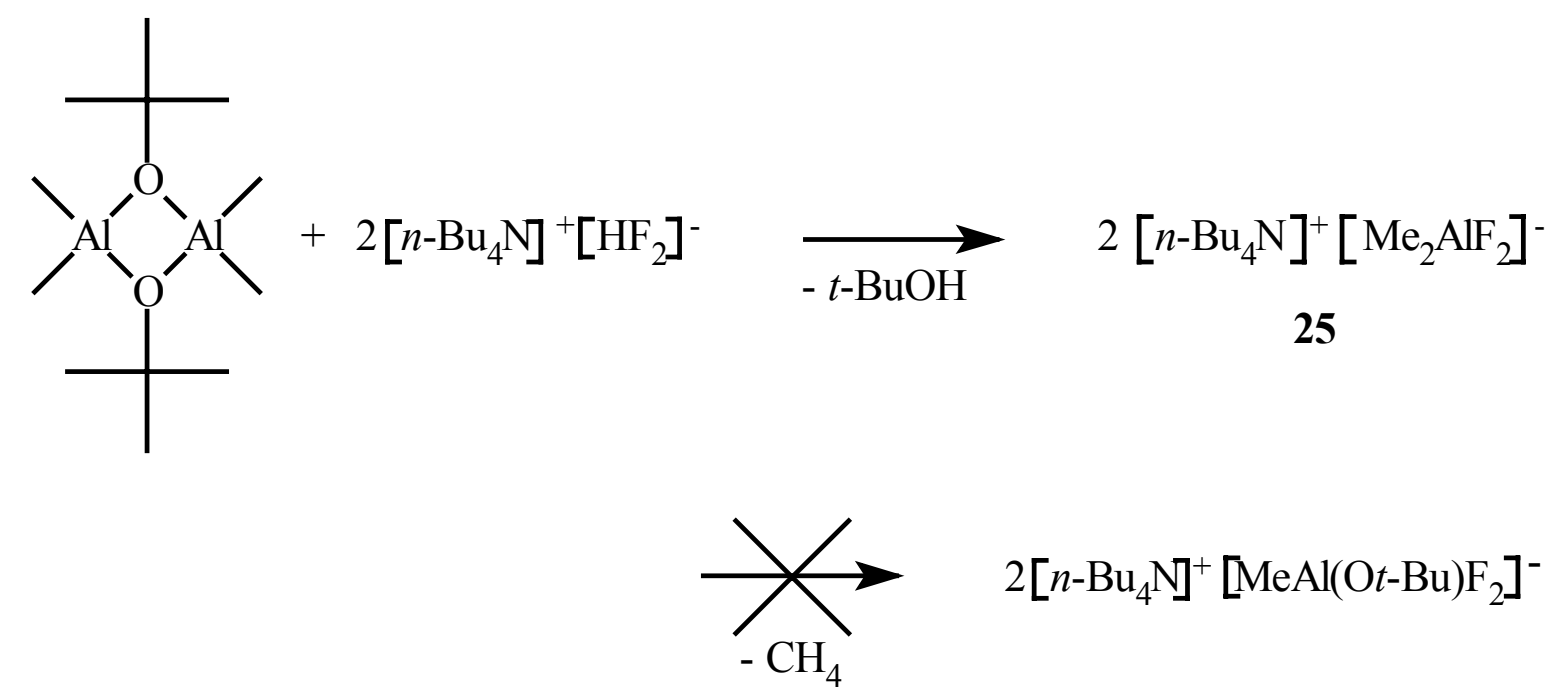

\subsubsection{Alkylsubstitution}

Die Fluorierung von Metallalkylen der 13. Gruppe wurde bereits untersucht (Kap. 2.4.1.) [110]. Um zu überprüfen, inwieweit sich dieses Reaktionsschema auf Übergangsmetallverbindungen übertragen läßt, wurde $\mathrm{Cp}_{2} \mathrm{ZrMe}_{2}$ mit $\mathrm{TBADF}$ und $\mathrm{KHF}_{2}$ jeweils in einem molaren Verhältnis von $1: 1$ umgesetzt. Im ${ }^{1} \mathrm{H}-\mathrm{NMR}-$ Spektrum des öligen Produkts der Reaktion mit TBADF findet man sowohl Signale von Protonen der Cp- als auch von Methylgruppen. Die Integrale sind im Vergleich zu denen der Protonen der $\left[n-\mathrm{BuN}_{4}\right]^{+}-$ Ionen viel zu klein, was darauf hinweist, daß kein bevorzugtes Produkt gebildet wurde. Daraufhin wurde die Reaktion mit einem doppelten Überschuß an $\mathrm{Cp}_{2} \mathrm{ZrMe}_{2}$ wiederholt. Das ${ }^{1}$ H-NMR-Spektrum ist identisch mit dem bei äquimolarem Einsatz. Eine mögliche Erklärung 
ist, daß das Reaktionsprodukt die Zersetzung des Edukts fördert. Das Signal im ${ }^{19}$ F-NMRSpektrum bei $\delta-12.0 \mathrm{ppm}$ weist auf endständige F-Atome hin, und läßt sich keiner literaturbekannten Verbindung zuordnen. Bei dem Versuch, $\mathrm{Cp}_{2} \mathrm{ZrMe}_{2}$ mit $\mathrm{KHF}_{2}$ zur Reaktion zu bringen, konnte selbst in siedendem Toluol keine Reaktion beobachtet werden.

Außerdem wurden $\mathrm{Cp}_{2} \mathrm{Ca}, \mathrm{Cp}_{2} \mathrm{Mg}, \mathrm{Me}_{2} \mathrm{Zn}$ und $\mathrm{Bis}_{2} \mathrm{Zn}$ mit TBADF zur Reaktion gebracht. Im allen Fällen lieferten die ${ }^{1} \mathrm{H}-\mathrm{NMR}-$ Spektren der Produkte für die Integrale der Protonensignale der Cp- bzw. Alkylgruppen zu kleine Werte. Die Produkte müssen sich während der Reaktion zersetzt haben.

\subsubsection{Hydridsubstitution}

Ein interessantes und kommerziell erhältliches Edukt für die Fluorierung durch TBADF ist das Schwartz'sche Reagens $\mathrm{Cp}_{2} \mathrm{Zr}(\mathrm{Cl}) \mathrm{H}$, welches bei Hydrozirconierungsreaktionen Verwendung findet [125]. Es wurde bereits versucht, Zirconocenchloridhydrid mit Trimethylzinnfluorid zu fluorieren [126]. Dabei erfolgte sowohl eine Substitution des Chlorids als auch des Hydrids, wobei aus dem entstandenen Produktgemisch kein einheitliches Produkt zu isolieren war. Um die Anwendbarkeit von TBADF für eine selektive Hydridsubstitution zu testen, wurde $\mathrm{Cp}_{2} \mathrm{Zr}(\mathrm{Cl}) \mathrm{H}$ mit $\left[n-\mathrm{Bu}_{4} \mathrm{~N}\right]^{+}\left[\mathrm{HF}_{2}\right]^{-}$bei $0{ }^{\circ} \mathrm{C}$ in einem molaren Verhältnis von $1: 1$ zur Reaktion gebracht. Unter sofort einsetzender Gasentwicklung löste sich die polymere und dadurch unlösliche Zirconiumverbindung auf und es entstand eine homogene Lösung. Nach Abtrennen des Lösungsmittels resultierte ein farbloses Öl. Das ${ }^{1}$ H-NMR-Spektrum des Rohprodukts weist ausschließlich Signale von Protonen des $[n-\mathrm{BuN}]^{+}$-Ions auf. Das ${ }^{19} \mathrm{~F}-\mathrm{NMR}-$ Spektrum bietet keinen Hinweis auf $\mathrm{Zr}-\mathrm{F}$ Bindungen, weshalb auf eine weitere Identifizierung des Produkts verzichtet wurde.

Als nächstes soll die Wasserstoffabspaltung bei Silanen untersucht werden. Wie bereits erwähnt, konnten Silane mit TBADF fluoriert werden. Diesem Reaktionsweg folgend, wurde $\mathrm{Ph}_{3} \mathrm{SiH}$ mit $\left[n-\mathrm{Bu}_{4} \mathrm{~N}\right]^{+}\left[\mathrm{HF}_{2}\right]^{-}$in einem molaren Verhältnis von $1: 1 \mathrm{zu}$ $\left[n-\mathrm{Bu}_{4} \mathrm{~N}\right]^{+}\left[\mathrm{Ph}_{3} \mathrm{SiF}_{2}\right]^{-}$(26) umgesetzt. Die Analytik liefert mit den Literaturwerten [105] übereinstimmende Daten. Aus einem Lösungsmittelgemisch (n-Hexan:Toluol 10:1) konnten über Nacht bei $-30^{\circ} \mathrm{C}$ für eine Strukturanalyse geeignete Kristalle gewonnen werden. Abbildung 12 zeigt die Struktur des Anions von Verbindung 26 im Festkörper und charakteristische Bindungslängen und -winkel sind in Tabelle 16 aufgeführt. 


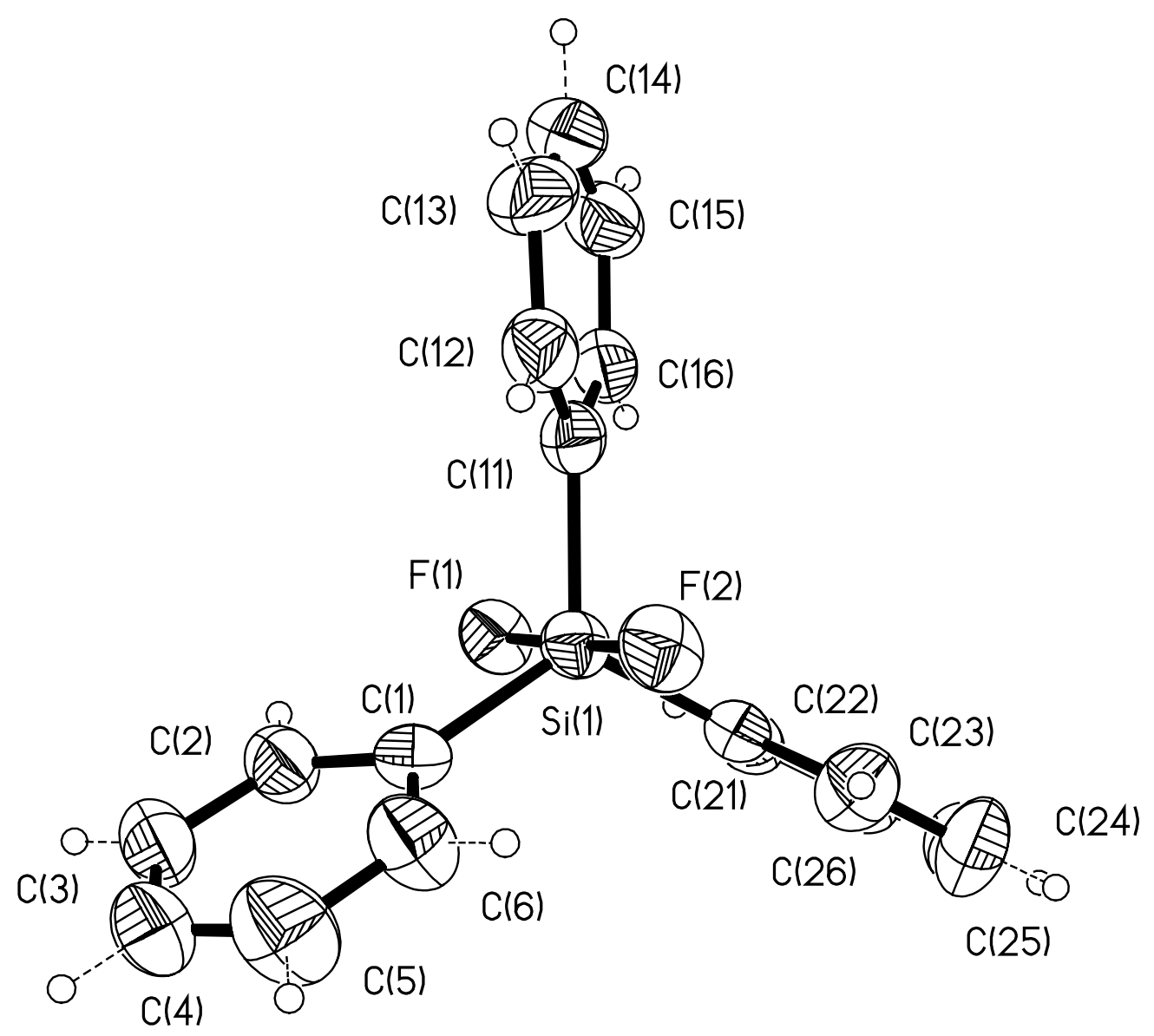

Abbildung 12: Struktur des Anions $\left(\mathrm{Ph}_{3} \mathrm{SiF}_{2}\right)^{-}$

Tabelle 16: Charakteristische Bindungslängen (pm) und -winkel $\left(^{\circ}\right)$ des Anions von

Verbindung 26

$\begin{array}{llll}\operatorname{Si}(1)-\mathrm{F}(1) & 172.2(3) & \mathrm{F}(1)-\operatorname{Si}(1)-\mathrm{F}(2) & 178.4(2) \\ \operatorname{Si}(1)-\mathrm{F}(2) & 173.2(3) & \mathrm{F}(1)-\operatorname{Si}(1)-\mathrm{C}(11) & 89.1(2) \\ \operatorname{Si}(1)-\mathrm{C}(1) & 190.7(5) & \mathrm{F}(1)-\operatorname{Si}(1)-\mathrm{C}(1) & 90.2(2) \\ \operatorname{Si}(1)-\mathrm{C}(11) & 190.8(5) & \mathrm{F}(1)-\operatorname{Si}(1)-\mathrm{C}(21) & 90.2(2) \\ \operatorname{Si}(1)-\mathrm{C}(21) & 191.1(6) & & \end{array}$


Verbindung 26 kristallisiert orthorhombisch in der Raumgruppe Pna2 ${ }_{1}$. Das Anion bildet eine trigonale Bipyramide aus. Aufgrund des größeren Platzbedarfs der Phenylgruppen gegenüber den Fluoratomen ordnen sie sich in der Äquatorialebene an.

\subsubsection{Weitere Substitutionsmöglichkeiten}

Rheniumbromide konnten mit $\mathrm{AgHF}_{2}$ bereits fluoriert werden [127, 128]. Deswegen war es interessant zu überprüfen, ob TBADF ebenfalls in der Lage ist, andere Halogenatome durch Fluor $\mathrm{zu}$ ersetzen. $\mathrm{AgHF}_{2}$ bietet gegenüber TBADF den Vorteil, das abzuspaltende Halogenid durch Fällung aus dem Gleichgewicht zu entfernen. Dafür ist die Löslichkeit von TBADF und damit die Konzentration in der Lösung deutlich höher als bei $\mathrm{AgHF}_{2}$. Um zu überprüfen, inwieweit sich TBADF für die Halogensubstitution eignet, wurden $\left(\mathrm{PPh}_{3}\right)_{2} \mathrm{MCl}_{2}$ $(\mathrm{M}=\mathrm{Co}, \mathrm{Ni})$ mit $[n-\mathrm{BuN}]^{+}\left[\mathrm{HF}_{2}\right]^{-}$umgesetzt. In beiden Fällen konnte keine Reaktion beobachtet werden. Offensichtlich ist das Entfernen des Halogenids, beispielsweise durch eine Fällung mit Silberionen, für den Fortgang der Reaktion förderlich.

Ein weiterer Ansatz für Fluorierungen mit TBADF ist die Substitution von Acetaten unter Essigsäureabspaltung. Als Ausgangsverbindungen wurden $\mathrm{Ph}_{3} \mathrm{Bi}(\mathrm{OAc})_{2}$ und $\left(\mathrm{PPh}_{3}\right)_{2} \operatorname{Ir}(\mathrm{OAc})_{2}$ gewählt. Beim Einsatz der Iridiumverbindung wurde ein schwarzes Öl isoliert, das in allen gängigen Lösungsmitteln unlöslich war. Offensichtlich zersetzte sich die Iridiumverbindung unkontrolliert. Die Bismutverbindung wurde mit TBADF in einem äquimolaren Verhältnis bei Raumtemperatur in THF umgesetzt. Nach ca. 15 min. war das unlösliche Bismutacetat fast vollständig abreagiert, was an der Auflösung des Niederschlags zu erkennen war. Eine geringe Menge des Bodensatzes wurde abfiltriert und das Filtrat bei $-30{ }^{\circ} \mathrm{C}$ zum Kristallisieren gestellt. Das Kristallisat wurde aufgrund seines Schmelzpunkts und ${ }^{1} \mathrm{H}-\mathrm{NMR}-$ Spektrums als Tetran-butylammoniumacetat identifiziert. Mit Hilfe von NMRspektroskopischen Untersuchungen der Mutterlauge konnte $\mathrm{Ph}_{3} \mathrm{BiF}_{2}$ nachgewiesen werden, eine Abtrennung gelang jedoch nicht. Mit diesem Versuch konnte gezeigt werden, daß es prinzipiell möglich ist, Acetatkomplexe mit TBADF zu fluorieren. Dabei wurde die Bildung des Beiprodukts Tetrabutylammoniumacetat beobachtet, das bei der Aufarbeitung Trennprobleme verursacht.

Acetylacetonat-Komplexe kommen als Reaktionspartner für TBADF in Frage, weil es hier leicht möglich ist, den Acetylacetonat-Liganden nach Protonierung zu verdrängen. Als Ausgangsmaterialien wurden $\mathrm{VO}(\mathrm{acac})_{2}$ und $\mathrm{MoO}_{2}(\text { acac })_{2}$ verwendet. Durch Umsatz mit 
einem Äquivalent $\left[n-\mathrm{Bu}_{4} \mathrm{~N}\right]^{+}\left[\mathrm{HF}_{2}\right]^{-}$konnte ein Acetylacetonat-Ligand durch zwei Fluoridionen ersetzt werden.

$$
\begin{array}{ccc}
\mathrm{VO}(\mathrm{acac})_{2}+\left[n-\mathrm{Bu}_{4} \mathrm{~N}\right]^{+}\left[\mathrm{HF}_{2}\right]^{-} & \underset{-\mathrm{H}(\mathrm{acac})}{\left[n-\mathrm{Bu}_{4} \mathrm{~N}\right]^{+}\left[\mathrm{VO}(\mathrm{acac}) \mathrm{F}_{2}\right]^{-}} \\
\mathrm{MoO}_{2}(\mathrm{acac})_{2}+\left[n-\mathrm{Bu}_{4} \mathrm{~N}\right]^{+}\left[\mathrm{HF}_{2}\right]^{-} & \begin{array}{c}
\mathbf{2 7} \\
-\mathrm{H}(\mathrm{acac})
\end{array} & {\left[n-\mathrm{Bu}_{4} \mathrm{~N}\right]^{+}\left[\mathrm{MoO}_{2}(\mathrm{acac}) \mathrm{F}_{2}\right]^{-}} \\
\mathbf{2 8}
\end{array}
$$

Die Produkte wurden NMR-spektroskopisch und massenspektrometrisch untersucht. In den ${ }^{1}$ H-NMR-Spektren der Molybdänverbindung liegen die Signale der Protonen der Tetrabutylammonium- und der Acetylacetonat-Moleküle im korrekten Intensitätsverhältnis vor. Aufgrund des Paramagnetismus der Vanadiumverbindung sind die Signale hier stark verbreitert, so daß sich keine Aussage über die Intensitäten machen läßt. In den ${ }^{19} \mathrm{~F}-\mathrm{NMR}$ Spektren findet man Signale bei $\delta-57.8$ ppm für 28 und $-28.0 \mathrm{ppm}$ für 27 die auf Metall-Fluor-Bindungen hinweisen. FAB-Massenspektren untermauern die Existenz der Zielverbindungen durch Peaks bei den erwarteten $\mathrm{m} / \mathrm{z}$-Verhältnissen mit korrekten Isotopenverteilungen. Da beide Verbindungen als Öle anfielen, schlug die Kristallisation fehl. Um eine Zielverbindung $\mathrm{zu}$ erhalten, die in fester Form vorliegt, wurden die Ausgangsmaterialien variiert. $\mathrm{Zu}$ diesem Zweck wurde $\mathrm{Zr}(\mathrm{acac})_{4}$ mit TBADF in einem molaren Verhältnis von $1: 1$ in THF zur Reaktion gebracht. Hierbei wurde ebenfalls ein Ö1 isoliert, das sich im Gegensatz zu 27 und 28 als in allen gängigen Lösungsmitteln unlöslich erwies. Auch die Umsetzung von $\mathrm{Zr}(\mathrm{acac})_{4}$ mit $\mathrm{KHF}_{2}$ in Toluol führte trotz mehrstündigem Erhitzen $\mathrm{zu}$ keiner Reaktion und das Zirconiumacetylacetonat wurde unverändert zurückgewonnen.

In diesen Reaktionen spielt die Länge der Alkylgruppen am Stickstoffatom des Fluorierungsmittels eine wichtige Rolle. Einerseits verleiht eine große Kettenlänge dem Fluorierungsmittel seine Löslichkeit in organischen Lösungsmitteln, andererseits führen sie zur Bildung von öligen Produkten. Wählt man als Substituenten die Ethylgruppe, lassen sich optimale Ergebnisse erzielen. Auf diesem Weg wurden die Feststoffe $\left[\mathrm{Et}_{4} \mathrm{~N}\right]^{+}\left[\mathrm{VO}(\mathrm{acac}) \mathrm{F}_{2}\right]^{-}$ und $\left[\mathrm{Et}_{4} \mathrm{~N}\right]^{+}\left[\mathrm{MoO}_{2}(\mathrm{acac}) \mathrm{F}_{2}\right]^{-}$isoliert [129]. Die Acetylacetonatsubstitution durch Hydrogendifluoride eröffnet einen Syntheseweg für eine Vielzahl neuer und interessanter Verbindungen. 


\section{Zusammenfassung}

Ziel der vorliegenden Arbeit war die Darstellung neuer Organometall-FluorVerbindungen unter Einbeziehung alternativer Fluorierungsmittel.

Im ersten Teil der Arbeit wurden Alkylierungsreaktionen mit $\mathrm{Bis}_{2} \mathrm{Zn}$ vorgestellt. Auf diesem Weg konnten die neuen Komplexe $\operatorname{BisMX}_{4}(\mathrm{M}=\mathrm{Ta}(\mathbf{1}), \mathrm{Nb}$ (4) für $\mathrm{X}=\mathrm{Cl}$ und $\mathrm{M}=$ $\mathrm{Ta}$ (2), $\mathrm{Nb}(5)$ für $\mathrm{X}=\mathrm{Br}$ ) synthetisiert werden. Die röntgenstrukturelle Charakterisierung von 1 und 4 zeigt monomere Molekülstrukturen mit trigonal-bipyramidal koordinierten Metallatomen.

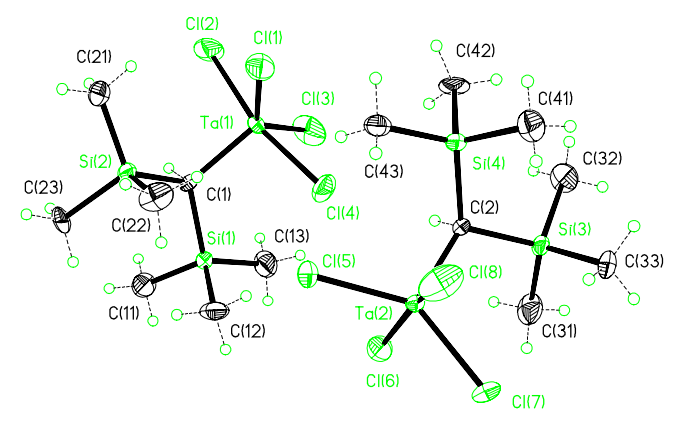

1

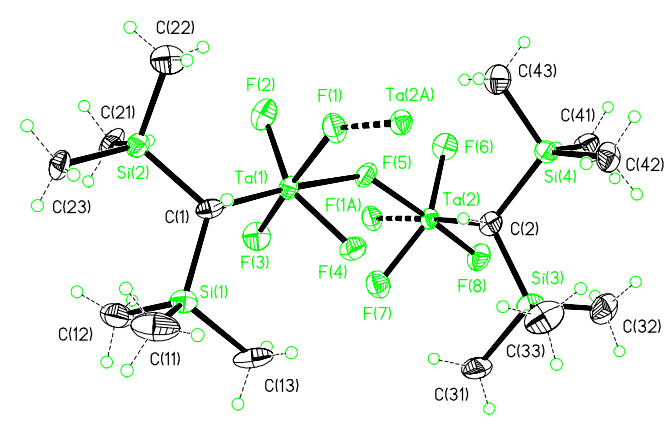

9

Ausgehend von den Chloro-Komplexen wurden die Komplexe $\mathrm{BisMF}_{4}$ (M = Ta (9), $\mathrm{Nb}$ (11)) mit Hilfe von $\mathrm{Me}_{3} \mathrm{SnF}$ über einen Chlor-Fluor-Austausch dargestellt. Mit Verbindung 11 wurde ein $\mathrm{C}-\mathrm{Nb}-\mathrm{F}-\mathrm{Fragment}$ aufgebaut, das eine $\mathrm{Nb}-\mathrm{C}-\sigma-\mathrm{Bindung}$ aufweist. Die entsprechende Tantalverbindung 9 stellt den ersten Vertreter eines monoalkylsubstituierten Tantalfluorids dar. Eine röntgenographische Untersuchung von Verbindung 9 ergab, daß sich die oktaedrisch koordinierten Metallzentren in Zickzackketten anordnen. Mit der Darstellung dieser beiden Verbindungen konnte das Substitutionsverhalten von $\mathrm{Me}_{3} \mathrm{SnF}$ gegenüber Alkylgruppen genauer untersucht werden. Dabei hat sich gezeigt, daß der sterische Anspruch des Liganden einen entscheidenden Einfluß hat. 
Als alternative und dem $\mathrm{Me}_{3} \mathrm{SnF}$ verwandte Fluorierungsmittel wurden $\mathrm{Ph}_{2} \mathrm{PbF}_{2}$ (13) und $\mathrm{Ph}_{3} \mathrm{BiF}_{2}$ (14) mit Metallocenkomplexen der vierten Gruppe $\mathrm{zu}$ den entsprechenden Fluorokomplexen umgesetzt. Es war das erste Mal, daß Blei- und Bismutfluoride für die metathetische Fluorierung eingesetzt wurden. Als Ausgangsverbindungen wurden neben $\mathrm{Cp}^{*} \mathrm{TiCl}_{3}, \mathrm{Cp}_{2} \mathrm{ZrCl}_{2}$ und $\mathrm{Cp}_{2} \mathrm{HfCl}_{2}$ auch die bislang unbekannten Verbindungen $\mathrm{Cp}^{*} \mathrm{Cp}^{\prime} \mathrm{MCl}_{2}$ ( $\mathrm{M}=\mathrm{Zr}$ (15), $\mathrm{Hf}(\mathbf{1 6}))$ gewählt. Die Chloro- und Fluorokomplexe wurden röntgenstrukturell charakterisiert. Alle Verbindungen liegen monomer mit verzerrt tetraedrisch koordinierten Metallzentren vor.

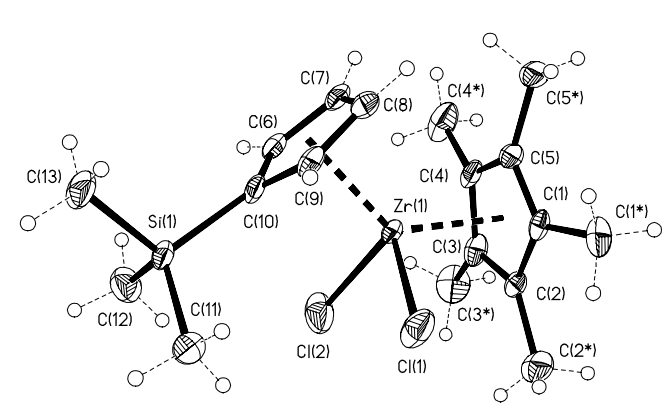

15

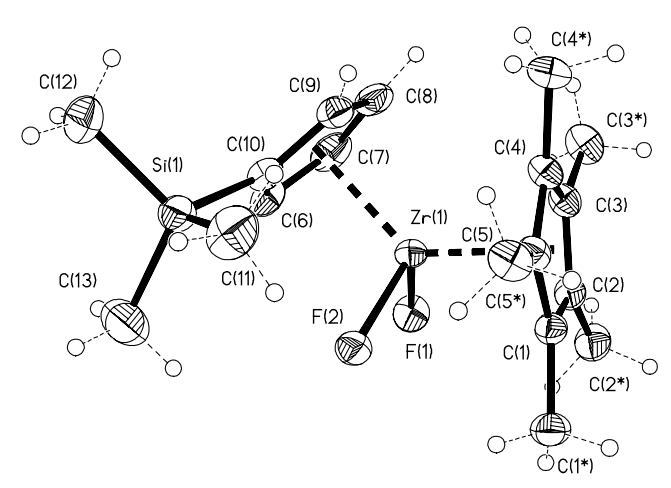

17

Fluorierungsmittel vom Typ $\mathrm{Q}^{+} \mathrm{HF}_{2}^{-}\left(\mathrm{Q}^{+}=n-\mathrm{Bu}_{4} \mathrm{~N}^{+} ; \mathrm{Et}_{4} \mathrm{~N}^{+} ; \mathrm{K}^{+}\right)$wurden bislang für die Synthese kaum erschlossen. Sie reagieren mit Metallalkoxiden unter Alkoholabspaltung zu komplex aufgebauten Metallclustern. Eine Vorhersage über den Reaktionsweg gestaltet sich oftmals schwierig, weil Etherabspaltungen als Nebenreaktionen postuliert werden kann. Meist gibt erst eine Einkristallröntgenstrukturanalyse Aufschluß über die Konstitution der Produkte. Auf diesem Weg gelang die Charakterisierung eines großen Aggregats, bestehend aus 8 Ta-Metallzentren, 20 Ethoxidresten und 10 Sauerstoffatomen, das in einer Reaktion von $n-\mathrm{Bu}_{4} \mathrm{NHF}_{2}$ mit $\mathrm{Ta}(\mathrm{OEt})_{5}$ dargestellt werden konnte. Die punktsymmetrische Struktur von Verbindung 23 besitzt im Zentrum einen achtgliedrigen Ring, der von sechsgliedrigen Ringen umgeben ist. 


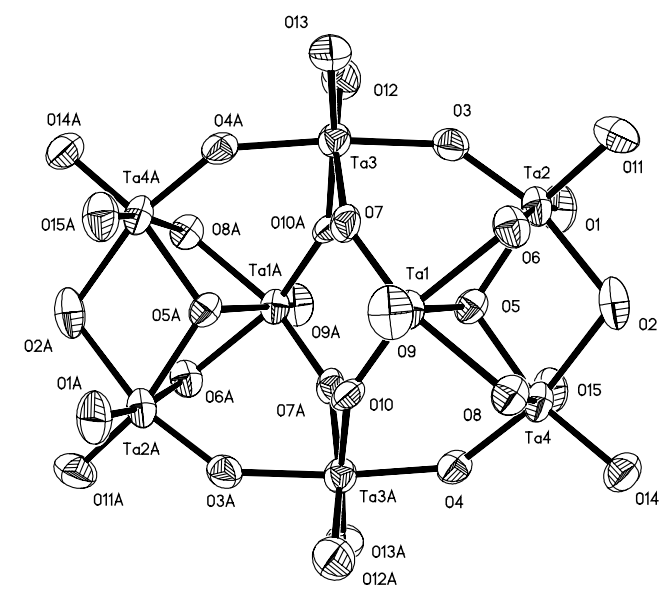

23

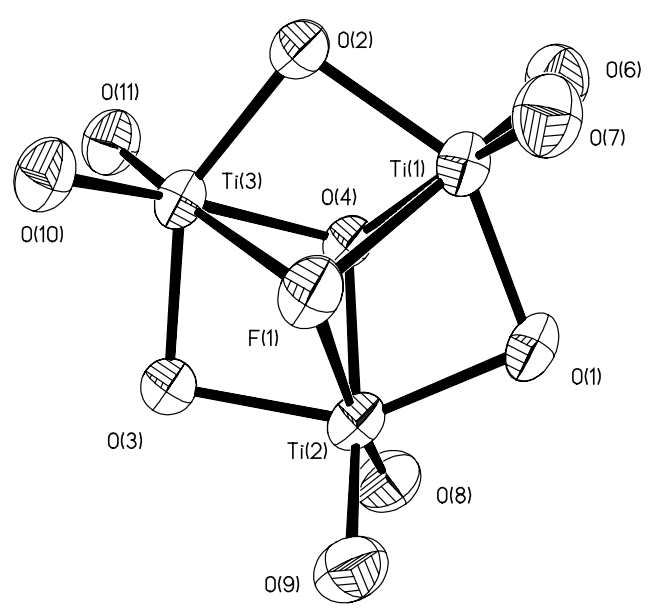

20

Die Reaktion von $\mathrm{Ti}(\mathrm{O} i \text {-Pr })_{4}$ mit $\mathrm{KHF}_{2}$ liefert ein ganz anderes Ergebnis. Nach Substitution eines Alkoxids durch ein Fluorid und einer Etherabspaltung erhält man Verbindung 20. Eine röntgenstrukturelle Analyse zeigt, daß ein sechsgliedriger Ring aus Alkoxidmolekülen und Titan aufgebaut wurde, in dessen Zentrum je ein Sauerstoff- und Fluoratom positioniert sind.

Neben den Reaktion von Alkoxiden mit $\mathrm{Q}^{+} \mathrm{HF}_{2}^{-}$ist die Fluorierung von Acetaten, Hydriden, Alkylderivaten und Acetylacetonaten in dieser Arbeit untersucht worden. Bei den Reaktionen mit Acetaten entsteht als Nebenpodukt $\mathrm{Q}^{+} \mathrm{OAc}{ }^{-}$, dessen Abtrennung Probleme bereitet. Hydrid- und Alkylderivate von Hauptgruppenelementen konnten erfolgreich mit TBADF fluoriert werden. Reaktionen an entsprechenden Verbindungen der Nebengruppen führten zu uneinheitlichen Produkten. Die Substitution von Acetylacetonat gegen zwei Fluoratome war erfolgreich. Die Acetylacetonate $\mathrm{VO}(\mathrm{acac})_{2}$ und $\mathrm{MoO}_{2}(\mathrm{acac})_{2}$ konnten mit TBADF erfolgreich fluoriert werden. Die weitere Erforschung des Synthesepotentials der hydrogenbifluoridhaltigen Fluorierungsmittel wird in naher Zukunft zu einer Vielzahl von interessanten Fluorokomplexen führen. 


\section{Experimenteller Teil}

\subsection{Allgemeine Arbeitstechniken}

Alle Reaktionen wurden in ausgeheizten und im Vakuum abgekühlten Glasgeräten unter einer getrockneten und sauerstofffreien Stickstoffatmosphäre durchgeführt. Die verwendeten Lösungsmittel wurden nach Literaturvorschriften getrocknet [130].

Schmelzpunkte wurden in unter Inertgas zugeschmolzenen Glaskapillaren mit den Geräten Bühler SPA-1 und Büchi B-540 gemessen. Die Bestimmung erfolgte ohne Temperaturkorrektur.

Elementaranalysen wurden vom Analytischen Labor des Institutes für Anorganische Chemie der Universität Göttingen durchgeführt.

NMR-Spektren wurden mit den Geräten Bruker AM 200 und Bruker Avance 500 aufgenommen. Chemische Verschiebungen sind in ppm angegeben, wobei positive Vorzeichen Tieffeldverschiebung in Bezug auf den jeweiligen Standard bedeuten. Als externe Standards dienten: ${ }^{1} \mathrm{H}$ : TMS; ${ }^{13} \mathrm{C}$ : TMS $;{ }^{19} \mathrm{~F}$ : $\mathrm{CFCl}_{3} ;{ }^{27} \mathrm{Al}: \mathrm{AlCl}_{3} ;{ }^{29} \mathrm{Si}$ : TMS und ${ }^{133} \mathrm{Cs}$ : CsF.

Die Multiplizitäten wurden wie folgt angegeben: $s=$ Singulett, $d=$ Dublett, $\mathrm{t}=$ Triplett, $\mathrm{q}=$ Quintett, sept $=$ Septett, $\mathrm{m}=$ Multiplett, $\mathrm{br}=$ breit. Alle Messungen erfolgten bei Raumtemperatur. Die Heterokern-NMR-Spektren sind außer den ${ }^{19}$ F-NMR-Spektren ${ }^{1}$ H-breitbandentkoppelt.

Alle Massenspektren wurden mit den Geräten Finnigan MAT 8200 und Finnigan MAT 95 aufgenommem. Als Ionisationsmethode wurde Elektronenstoßionisation (EI) bei $70 \mathrm{eV}$ verwendet. Als Matrix bei den FAB-MS-Messungen diente 3-Nitrobenzylalkohol.

IR-Spektren wurden als Nujolverreibungen zwischen CsI-Platten auf einem BIO-RAD Digilab FTS 7 Spektrometer aufgenommen. Die Bandenintensitäten werden wie folgt abgekürzt: sst $=$ sehr stark, $s t=$ stark, $\mathrm{m}=$ mittel, $\mathrm{s}=$ schwach . 
Die Einkristall-Röntgenstrukturanalyse wurde auf einem Stoe-SiemensVierkreisdiffraktometer mit graphitmonochromatisierter Mo- $\mathrm{K}_{\alpha}-$ Strahlung $(\lambda=71.073 \mathrm{pm})$ durchgeführt. Die Strukturen wurden nach der Methode der kleinsten Fehlerquadrate mit voller Matrix $F^{2}$ verfeinert. Dafür wurde das Programm SHELX-97 [131] verwendet.

\subsection{Darstellung der Ausgangsverbindungen}

Folgende Reagenzien wurden gemäß den angegebenen Literaturmethoden synthetisiert: $\quad t$ - $\mathrm{Bu}_{3} \mathrm{SiOH}[132] ; \quad t$ - $\mathrm{Bu}_{3} \mathrm{SiONa}[133] ; \quad\left(t-\mathrm{Bu}_{3} \mathrm{SiO}_{3} \mathrm{TaCl}_{2}[70]\right.$; $\left(\mathrm{Me}_{3} \mathrm{SiCH}_{2}\right)_{2} \mathrm{Zn}$ [134], $\quad\left(\mathrm{Me}_{3} \mathrm{SiCH}_{2}\right)_{3} \mathrm{TaCl}_{2}$ [134]; $\quad\left(\mathrm{Me}_{3} \mathrm{Si}\right)_{2} \mathrm{CHBr}$ [135]; $\left[\left(\mathrm{Me}_{3} \mathrm{Si}\right)_{2} \mathrm{CH}\right]_{2} \mathrm{Zn}[54] ; \quad \mathrm{Me}_{3} \mathrm{SnF}$ [136]; $\mathrm{Ph}_{2} \mathrm{PbF}_{2}$ [137]; $\mathrm{Ph}_{3} \mathrm{BiF}_{2}$ [138]; $\mathrm{Ph}_{3} \mathrm{Bi}(\mathrm{OAc})_{2}$ [139]; Cp* $\mathrm{TiCl}_{3}$ [140]; $\mathrm{Cp}^{*} \mathrm{Cp}^{\prime} \mathrm{ZrCl}_{2}$ [90]; $\mathrm{Cp}_{2} \mathrm{ZrMe}_{2}$ [141] $\mathrm{Cp}^{*} \mathrm{TaCl}_{4}[142] ; \quad\left(\mathrm{Cp}^{*} \mathrm{CrBr}_{2}\right)_{2}$ [143]; $\mathrm{Cp}^{*} \mathrm{MoCl}_{4}$ [144]; $\left(\mathrm{Cp} * \mathrm{RuCl}_{2}\right)_{2}$ [145]; $\mathrm{Cp}_{2} \mathrm{Mg}$ [146]; $\mathrm{Cp}_{2} \mathrm{Ca}$ [147]; $\left(t-\mathrm{BuOAlMe}_{2}\right)_{2}$ [148]. 


\subsection{Durchführung der Experimente}

\subsection{1. $\mathrm{BisTaCl}_{4}(1)$}

$\mathrm{Zu}$ einer Suspension von $\mathrm{TaCl}_{5}(7.0 \mathrm{~g}, 19.5 \mathrm{mmol})$ in $n$-Hexan $(100 \mathrm{ml})$ wurde tropfenweise $\mathrm{Bis}_{2} \mathrm{Zn}(3.0 \mathrm{~g}, 7.8 \mathrm{mmol})$ bei $-70^{\circ} \mathrm{C}$ zugegeben. Beim langsamen Erwärmen auf Raumtemperatur färbte sich das Gemisch orange und während 2 stündigem Rühren gelb. Das ausgefallene $\mathrm{ZnCl}_{2}$ und überschüssiges $\mathrm{TaCl}_{5}$ wurden unter Verwendung von Celite abfiltriert. Die Lösung wurde im Vakuum auf ca. $30 \mathrm{ml}$ eingeengt und bei $-70{ }^{\circ} \mathrm{C}$ über Nacht kristallisiert, wobei 1 in Form von nadelförmigen, gelben Kristallen ausfiel. Ausbeute $4.9 \mathrm{~g}$ $(65 \%)$.

Schmelzpunkt: $68^{\circ} \mathrm{C}$.

Elementaranalyse für $\mathrm{C}_{7} \mathrm{H}_{19} \mathrm{Cl}_{4} \mathrm{Si}_{2} \mathrm{Ta}(482.16)$ :

$\begin{array}{llll} & \mathrm{C} & \mathrm{H} & \mathrm{Cl} \\ \text { Ber. (\%): } & 17.44 & 3.97 & 29.41, \\ \text { Gef. (\%): } & 17.8 & 4.1 & 29.1 .\end{array}$

${ }^{1} \mathrm{H}-\mathrm{NMR}-$ Spektrum (200 MHz, $\left.\mathrm{CDCl}_{3}\right): \delta 0.45\left(\mathrm{~s}, 18 \mathrm{H}, \mathrm{CH}_{3}\right), 4.65(\mathrm{~s}, 1 \mathrm{H}, \mathrm{CH}) \mathrm{ppm}$.

${ }^{13} \mathrm{C}-\mathrm{NMR}-$ Spektrum $\left(63 \mathrm{MHz}, \mathrm{CDCl}_{3}\right): \delta 4.2\left(\mathrm{CH}_{3}\right), 146.3(\mathrm{CH}) \mathrm{ppm}$.

${ }^{29} \mathrm{Si}-\mathrm{NMR}-\mathrm{Spektrum}\left(50 \mathrm{MHz}, \mathrm{CDCl}_{3}\right): \delta 6.0\left(\mathrm{~s}, \mathrm{SiMe}_{3}\right) \mathrm{ppm}$.

Massenspektrum (EI) m/z (\%): [M+ $-\mathrm{Me}] 467$ (50), [ $\left.\mathrm{M}^{+}-\mathrm{SiMe}_{3}-\mathrm{Me}-\mathrm{Cl}\right] 359$ (100), $\left[\mathrm{M}^{+}-\mathrm{CH}\left(\mathrm{SiMe}_{3}\right)_{2}\right] 323(25),\left[\mathrm{SiMe}_{3}^{+}\right] 73(35)$.

IR (Nujol, CsI): $\widetilde{v} 1407$ (m), 1254 (st), 926 (m), 899 (st), 853 (sst), 836 (sst), 780 (st), $751(\mathrm{~m}), 696$ (st), 652 (st), 606 (m), 579 (s), 522 (s), 456 (m), 382 (m), 349 (s), 282 (s) $\mathrm{cm}^{-1}$. 


\subsection{2. $\mathrm{BisTaBr}_{4}(2)$}

Es wurde analog Arbeitsvorschrift 4.3.1. verfahren. $\operatorname{TaBr}_{5}(3.0 \mathrm{~g}, 5.2 \mathrm{mmol})$ wurde mit Bis $_{2} \mathrm{Zn}(0.8 \mathrm{~g}, 2.1 \mathrm{mmol})$ zu 2 umgesetzt, das in Form von orangen Nadeln anfiel. Ausbeute: $0.81 \mathrm{~g}(30 \%)$.

Schmelzpunkt: $79{ }^{\circ} \mathrm{C}$.

Elementaranalyse für $\mathrm{C}_{7} \mathrm{H}_{19} \mathrm{Br}_{4} \mathrm{Si}_{2} \mathrm{Ta}$ (660.04):<smiles>[1H]</smiles>

Ber. $(\%): \quad 12.74 \quad 2.90$,

Gef. $(\%)$ : $\quad 3.3$.

${ }^{1} \mathrm{H}-\mathrm{NMR}-$ Spektrum $\left(200 \mathrm{MHz}, \mathrm{CDCl}_{3}\right): \delta 0.52\left(\mathrm{~s}, 18 \mathrm{H}, \mathrm{CH}_{3}\right), 4.45(\mathrm{~s}, 1 \mathrm{H}, \mathrm{CH}) \mathrm{ppm}$.

${ }^{29} \mathrm{Si}-\mathrm{NMR}-\mathrm{Spektrum}\left(50 \mathrm{MHz}, \mathrm{CDCl}_{3}\right): \delta 2.5\left(\mathrm{~s}, \mathrm{SiMe}_{3}\right) \mathrm{ppm}$.

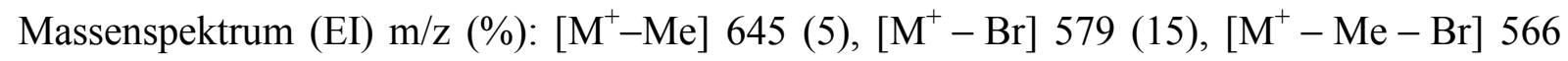
(5), $\left[\mathrm{M}^{+}-\mathrm{SiMe}_{3}-\mathrm{Br}\right] 506(40),\left[\mathrm{SiMe}_{3}{ }^{+}\right], 73$ (100).

IR (Nujol, CsI): $\tilde{v} 1405$ (m), 1264 (m), 1254 (st), 1251 (st), 1098 (s), 1024 (st), 927 (m), 888 (st), 852 (sst), 832 (sst), 778 (st), 751 (m), $692(\mathrm{~m}), 674$ (st), 646 (st), 632 (m), $603(\mathrm{~m}), 438(\mathrm{~s}), 332$ (s), 261 (st), $236(\mathrm{st}), 228(\mathrm{st}) \mathrm{cm}^{-1}$.

\subsection{3. $\mathrm{BisNbCl}_{4}(4)$}

Es wurde nach Vorschrift 4.3.1. verfahren. Aus $\mathrm{NbCl}_{5}(5.4 \mathrm{~g}, 20.0 \mathrm{mmol})$ und $\mathrm{Bis}_{2} \mathrm{Zn}$ ( $3.1 \mathrm{~g}, 8.1 \mathrm{mmol})$ wurde 4 in Form von orangen Kristallen in einer Ausbeute von $3.8 \mathrm{~g}$ (60\%) isoliert. 
Schmelzpunkt: $82^{\circ} \mathrm{C}$.

Elementaranalyse für $\mathrm{C}_{7} \mathrm{H}_{19} \mathrm{Cl}_{4} \mathrm{NbSi}_{2}$ (394.12):

$\begin{array}{llll} & \mathrm{C} & \mathrm{H} & \mathrm{Cl} \\ \text { Ber. (\%): } & 21.33 & 4.86 & 35.90, \\ \text { Gef. (\%): } & 21.5 & 5.0 & 35.7 .\end{array}$

${ }^{1} \mathrm{H}-\mathrm{NMR}-$ Spektrum $\left(200 \mathrm{MHz}, \mathrm{CDCl}_{3}\right): \delta 0.49\left(\mathrm{~s}, 18 \mathrm{H}, \mathrm{CH}_{3}\right), 6.20(\mathrm{~s}, 1 \mathrm{H}, \mathrm{CH}) \mathrm{ppm}$.

${ }^{13} \mathrm{C}-\mathrm{NMR}-$ Spektrum $\left(63 \mathrm{MHz}, \mathrm{CDCl}_{3}\right): \delta 3.8\left(\mathrm{CH}_{3}\right), 170.7(\mathrm{CH}) \mathrm{ppm}$.

${ }^{29} \mathrm{Si}-\mathrm{NMR}-\mathrm{Spektrum}\left(50 \mathrm{MHz}, \mathrm{CDCl}_{3}\right): \delta 9.4\left(\mathrm{~s}, \mathrm{SiMe}_{3}\right) \mathrm{ppm}$.

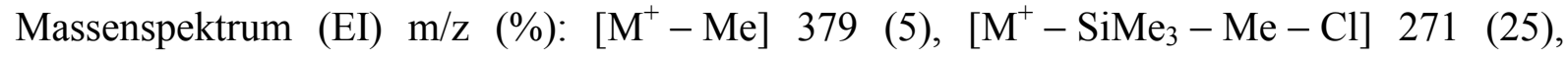
$\left[\mathrm{SiMe}_{3}^{+}\right] 73(100)$.

IR (Nujol, CsI): $\quad \widetilde{v} 1406$ (m), 1255 (st), $1156(\mathrm{w}), 1080$ (s), 943 (s), 906 (st), 879 (st), 853 (sst), 832 (sst), 791 (m), 777 (m), 751 (m), 698 (m), 669 (st), 637 (st), 601 (st), 445 (m), 336 (st), 369 (st), 333 (m), 280 (s) $\mathrm{cm}^{-1}$.

\subsection{4. $\mathrm{BisNbBr}_{4}(5)$}

Verbindung 5 wurde analog zu Arbeitsvorschrift 4.3.1. synthetisiert. $\mathrm{NbBr}_{5}(2.8 \mathrm{~g}$, $5.7 \mathrm{mmol})$ und $\mathrm{Bis}_{2} \mathrm{Zn}(0.7 \mathrm{~g}, 1.8 \mathrm{mmol})$ ergaben 5 in Form von orangeroten Kristallen. Ausbeute: $0.4 \mathrm{~g}(15 \%)$.

Schmelzpunkt: $91{ }^{\circ} \mathrm{C}$. 
Elementaranalyse für $\mathrm{C}_{7} \mathrm{H}_{19} \mathrm{Br}_{4} \mathrm{NbSi}_{2}$ (572.12):

$\begin{array}{lll} & \mathrm{C} & \mathrm{H} \\ \text { Ber. (\%): } & 14.70 & 3.35, \\ \text { Gef. }(\%): & 15.9 & 3.6 .\end{array}$

${ }^{1} \mathrm{H}-\mathrm{NMR}-$ Spektrum $\left(200 \mathrm{MHz}, \mathrm{CDCl}_{3}\right): \delta 0.53\left(\mathrm{~s}, 18 \mathrm{H}, \mathrm{CH}_{3}\right), 5.99(\mathrm{~s}, 1 \mathrm{H}, \mathrm{CH}) \mathrm{ppm}$.

${ }^{29} \mathrm{Si}-\mathrm{NMR}-\mathrm{Spektrum}\left(50 \mathrm{MHz}, \mathrm{CDCl}_{3}\right): \delta 5.5\left(\mathrm{~s}, \mathrm{SiMe}_{3}\right) \mathrm{ppm}$.

Massenspektrum (EI) m/z (\%): $\left[\mathrm{M}^{+}-\mathrm{Br}\right] 491(1),\left[\mathrm{M}^{+}-\mathrm{SiMe}_{3}-\mathrm{Br}\right] 418$ (3), $\left[\mathrm{SiMe}_{3}^{+}\right], 73$ $(100)$.

IR (Nujol, CsI): $\widetilde{v} 1405$ (m), 1253 (st), 1053 (s), 1040 (s), 940 (m), 909 (m), 849 (sst), $776(\mathrm{st}), 752$ (st), 669 (st), 638 (st), 624 (st), 596 (st), 446 (s), 423 (s), 331 (s), 302 (st), 275 (st), 261 (st), 238 (st) $\mathrm{cm}^{-1}$.

\subsection{5. $\mathrm{BisTiCl}_{3}(6)$}

$\mathrm{Zu}$ n-Hexan $(60 \mathrm{ml})$ wurden mit einer Spritze zuerst $\mathrm{TiCl}_{4}(5.0 \mathrm{~g}, 26 \mathrm{mmol})$ und dannach $\mathrm{Bis}_{2} \mathrm{Zn}(4.0 \mathrm{~g}, 10 \mathrm{mmol})$ bei $0{ }^{\circ} \mathrm{C}$ zugetropft. Das Reaktionsgemisch färbte sich sofort tiefrot. Nach zwei stündigem Rühren wurde das ausgefallene Zinkchlorid unter Verwendung von Celite abgetrennt. Nach Entfernen des Lösungsmittels im Vakuum wurde durch fraktionierende Destillation $\left(58^{\circ} \mathrm{C}, 0.02 \mathrm{mbar}\right)$ das Titanorganyl als orangefarbenes Öl isoliert. Ausbeute: $5.4 \mathrm{~g}(83 \%)$.

Siedepunkt: $58^{\circ} \mathrm{C}$ (0.02 mbar), Schmelzpunkt: ca. $-30{ }^{\circ} \mathrm{C}$.

Elementaranalyse für $\mathrm{C}_{7} \mathrm{H}_{19} \mathrm{Cl}_{3} \mathrm{Si}_{2} \mathrm{Ti}$ (313.64):

$\mathrm{C} \quad \mathrm{H} \quad \mathrm{Cl}$

$\begin{array}{lccc}\text { Ber. (\%): } & 26.81 & 6.11 & 33.91, \\ \text { Gef. (\%): } & 28.3 & 6.4 & 31.8 . \\ { }^{1} \mathrm{H}-\mathrm{NMR}-\text { Spektrum } & \left(200 \mathrm{MHz}, \mathrm{CDCl}_{3}\right): \delta 0.38(\mathrm{~s}, 18 \mathrm{H}, \mathrm{CH}), 6.29(\mathrm{~s}, 1 \mathrm{H}, \mathrm{CH}) \mathrm{ppm} .\end{array}$ 
${ }^{29} \mathrm{Si}-\mathrm{NMR}-\mathrm{Spektrum}\left(50 \mathrm{MHz}, \mathrm{CDCl}_{3}\right): \delta 3.4\left(\mathrm{~s}, \mathrm{SiMe}_{3}\right) \mathrm{ppm}$.

Massenspektrum (EI) m/z (\%): $\left[\mathrm{M}^{+}-\mathrm{Me}\right] 299$ (20), $\left[\mathrm{SiMe}_{3}{ }^{+}\right] 73$ (100).

IR (CsI): $\widetilde{v} 1254$ (st), 1087 (s), 1031 (s), 941 (m), 853 (sst), 832 (sst), 775 (m), 751 (m), 707 (m), 648 (m), 619 (m), 493 (st), 472 (st), 440 (m), 390 (m), 326 (s), 274 (s) $\mathrm{cm}^{-1}$.

\subsection{6. $\mathrm{BisTaF}_{4}(9)$}

$\mathrm{Me}_{3} \operatorname{SnF}(7.1 \mathrm{~g}, 40 \mathrm{mmol})$ wurde als Feststoff vorgelegt und mit einer auf $0{ }^{\circ} \mathrm{C}$ gekühlten Lösung von $\mathrm{BisTaCl}_{4}(4.7 \mathrm{~g}, 10 \mathrm{mmol})$ in $\mathrm{CH}_{2} \mathrm{Cl}_{2}(20 \mathrm{ml})$ versetzt. Während sich der Bodensatz innerhalb von 30 min auflöste, wechselte die Farbe des Reaktionsgemisches von gelb nach farblos. Nach Abtrennung aller flüchtigen Bestandteile im Vakuum über einen Zeitraum von $8 \mathrm{~h}$, wurde der Rückstand sublimiert $\left(90^{\circ} \mathrm{C}, 0.02 \mathrm{mbar}\right)$. Die Zielverbindung wurde als farbloser Feststoff in einer Ausbeute von $2.1 \mathrm{~g}(52 \%)$ isoliert.

Schmelzpunkt: $96{ }^{\circ} \mathrm{C}$.

Elementaranalyse für $\mathrm{C}_{7} \mathrm{H}_{19} \mathrm{~F}_{4} \mathrm{Si}_{2} \mathrm{Ta}$ (416.34):

$\begin{array}{llll} & \mathrm{C} & \mathrm{H} & \mathrm{F} \\ \text { Ber. (\%): } & 20.19 & 4.60 & 18.27, \\ \text { Gef. (\%): } & 19.9 & 4.7 & 17.9 .\end{array}$

${ }^{1} \mathrm{H}-\mathrm{NMR}-$ Spektrum $\left(200 \mathrm{MHz}, \mathrm{CDCl}_{3}\right): \delta 0.28(\mathrm{~s}, 18 \mathrm{H}, \mathrm{CH}), 3.09(\mathrm{~s}, 1 \mathrm{H}, \mathrm{CH}) \mathrm{ppm}$.

${ }^{13} \mathrm{C}-\mathrm{NMR}-$ Spektrum $\left(63 \mathrm{MHz}, \mathrm{CDCl}_{3}\right): \delta 2.5\left(\mathrm{CH}_{3}\right) ; 110.7(\mathrm{CH}) \mathrm{ppm}$.

${ }^{19}$ F-NMR-Spektrum (188 MHz, $\left.\mathrm{CDCl}_{3}\right): \delta 81.5$ (br) ppm.

${ }^{29} \mathrm{Si}-\mathrm{NMR}-\mathrm{Spektrum}\left(50 \mathrm{MHz}, \mathrm{CDCl}_{3}\right): \delta 5.54\left(\mathrm{~s}, \mathrm{SiMe}_{3}\right) \mathrm{ppm}$. 
Massenspektrum (EI) m/z (\%): [M+ $-\mathrm{Me}] 401$ (45), [M+ $\left.-\mathrm{SiMe}_{3}-\mathrm{Me}-\mathrm{F}\right] 309$ (100), $\left[\mathrm{SiMe}_{3}^{+}\right] 73(35)$.

IR (Nujol, CsI): $\widetilde{v} 1409$ (m), 1305 (st), 1254 (st), 955 (m), 920 (st), 864 (sst), 839 (sst), 784 (m), 757 (st), 723 (m), 704 (m), 688 (m), 643 (sst), 625 (st), 579 (s), 516 (st), 504 (st), 394 (s), 302 (s), 270 (s) $\mathrm{cm}^{-1}$.

\subsection{7. $\left[\left(\mathrm{BisTaF}_{4}\right)_{2} \mathrm{CsF}\right](10)$}

Die Feststoffe $\mathrm{BisTaF}_{4}(\mathbf{9})(0.5 \mathrm{~g}, 1.2 \mathrm{mmol})$ und CsF (0.09 g, $\left.0.60 \mathrm{mmol}\right)$ wurden bei Raumtemperatur mit THF $(10 \mathrm{ml})$ und $\mathrm{CH}_{2} \mathrm{Cl}_{2}(20 \mathrm{ml})$ versetzt. Dabei löste sich der Bodensatz fast vollständig auf. Unlösliche Bestandteile wurden durch Celite abfiltriert. Nach Abtrennung des Lösungsmittels im Vakuum erhält man die Zielverbindung als farblosen Feststoff: $0.5 \mathrm{~g},(85 \%)$.

Schmelzpunkt: $223^{\circ} \mathrm{C}$.

${ }^{19}$ F-NMR-Spektrum (188 MHz, THF/C $\left.6 \mathrm{D}_{6} 1: 1\right): \delta 45.7$ (br) ppm.

${ }^{133}$ Cs-NMR-Spektrum (33 MHz, THF/C $\left.6 \mathrm{D}_{6} 1: 1\right): \delta-55.5$ (s) ppm.

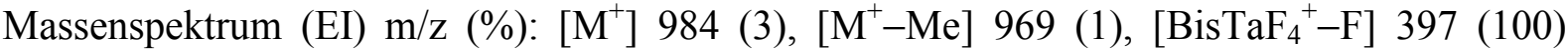
$\left[\mathrm{SiMe}_{3}^{+}\right] 73(40)$. 


\subsection{8. $\mathrm{BisNbF}_{4}(11)$}

$\mathrm{Zu}$ festem $\mathrm{Me}_{3} \mathrm{SnF}$ (7) (1.5 g, $\left.8.0 \mathrm{mmol}\right)$ wurde eine Lösung von $\mathrm{BisNbCl}_{4}$ (4) (0.8 g, $2.0 \mathrm{mmol})$ in $\mathrm{CH}_{2} \mathrm{Cl}_{2}(40 \mathrm{ml})$ bei $-70{ }^{\circ} \mathrm{C}$ gegeben. Beim Erwärmen auf Raumtemperatur ging der Feststoff in Lösung. Die Farbe wechselte dabei von orange nach gelb. Das Lösungsmittel und das Beiprodukt wurden unter Vakuum über einen Zeitraum von $8 \mathrm{~h}$ abgetrennt. Durch Sublimation des Rückstands bei $50{ }^{\circ} \mathrm{C}$ und 0.02 mbar ließ sich die Zielverbindung, $0.13 \mathrm{~g}$ (20 \%) als gelber Feststoff isolieren.

Zersetzungspunkt: $110^{\circ} \mathrm{C}$

Elementaranalyse für $\mathrm{C}_{7} \mathrm{H}_{19} \mathrm{~F}_{4} \mathrm{NbSi}_{2}$ (328.30):

$\begin{array}{llll} & \mathrm{C} & \mathrm{H} & \mathrm{F} \\ \text { Ber. (\%): } & 25.61 & 5.83 & 23.15, \\ \text { Gef. (\%): } & 25.6 & 5.7 & 22.9 .\end{array}$

${ }^{1} \mathrm{H}-\mathrm{NMR}-$ Spektrum $\left(200 \mathrm{MHz}, \mathrm{CDCl}_{3}\right): \delta 0.31(\mathrm{~s}, 18 \mathrm{H}, \mathrm{CH}), 4.65(\mathrm{~s}, 1 \mathrm{H}, \mathrm{CH}) \mathrm{ppm}$.

${ }^{19}$ F-NMR-Spektrum $\left(188 \mathrm{MHz}, \mathrm{CDCl}_{3}\right): \delta 126.0(\mathrm{~s}, \mathrm{Nb} F)$ ppm.

${ }^{29} \mathrm{Si}-\mathrm{NMR}-\mathrm{Spektrum}\left(50 \mathrm{MHz}, \mathrm{CDCl}_{3}\right): \delta 10.1\left(\mathrm{~s}, \mathrm{SiMe}_{3}\right) \mathrm{ppm}$.

Massenspektrum (EI) m/z (\%): [M+ - Me 313 (25), [M+ $\left.-\mathrm{SiMe}_{3}-\mathrm{Me}-\mathrm{F}\right] 221$ (100), $\left[\mathrm{SiMe}_{3}{ }^{+}\right] 73(50)$.

IR (Nujol, KBr): $\widetilde{v} 1255$ (st), 954 (m), 944 (m), 904 (st), 865 (st), 840 (st), 758 (m), 716 (m), $687(\mathrm{~m}), 661$ (sst), 624 (sst), $521(\mathrm{~m}), 509(\mathrm{~m}) \mathrm{cm}^{-1}$. 


\subsubsection{Allgemeine Vorschrift zur Fluorierung mit $\mathrm{Ph}_{2} \mathrm{PbF}_{2}(13)$ und $\mathrm{Ph}_{3} \mathrm{BiF}_{2}$ (14)}

Das jeweilige metallorganische Chlorid und das Fluorierungsmittel wurden in stöchiometrischem Verhältnis als Feststoffe eingewogen. Das Lösungsmittel $\mathrm{CH}_{2} \mathrm{Cl}_{2}(30 \mathrm{ml})$ wurde unter vermindertem Druck einkondensiert. Nach $12 \mathrm{~h}$ Rühren bei Raumtemperatur ließen sich weder Farbänderung noch Auflösen des Bodensatzes beobachten. Das $\mathrm{CH}_{2} \mathrm{Cl}_{2}$ wurde vollständig im Vakuum abgetrennt und die Rückstände wurden eine weitere Stunde evakuiert. Durch Sublimation $\left(90^{\circ} \mathrm{C}, 0.02\right.$ mbar) wurden die Produkte vom Beiprodukt abgetrennt.

\subsubsection{Darstellung von $\mathrm{Cp}^{*} \mathrm{TiF}_{3}, \mathrm{Cp}_{2} \mathrm{ZrF}_{2}$ undCp $\mathrm{p}_{2} \mathrm{HfF}_{2}$ jeweils mit $\mathrm{Ph}_{2} \mathrm{PbF}_{2}$ (13) und $\mathrm{Ph}_{3} \mathrm{BiF}_{2}(14)$}

Alle Fluorierungen erfolgten nach Arbeitsvorschrift 4.3.9. Die ${ }^{1} \mathrm{H}-$ und ${ }^{19} \mathrm{~F}-\mathrm{NMR}$ spektroskopische Charakterisierung stimmt mit den Literaturwerten überein. In Tabelle 17 sind die Produktmengen und Ausbeuten aufgeführt.

Fluorierungmittel/Produkt

$$
\mathrm{Cp}^{*} \mathrm{TiF}_{3}
$$

$\mathrm{Cp}_{2} \mathrm{ZrF}_{2}$

$\mathrm{Cp}_{2} \mathrm{HfF}_{2}$

$\begin{array}{llll}\mathrm{Ph}_{2} \mathrm{PbF}_{2} & 0.13 \mathrm{~g}(65 \%) & 0.06 \mathrm{~g}(27 \%) & 0.10 \mathrm{~g}(23 \%) \\ \mathrm{Ph}_{3} \mathrm{BiF}_{2} & 0.12 \mathrm{~g}(72 \%) & 0.09 \mathrm{~g}(40 \%) & 0.20 \mathrm{~g}(44 \%)\end{array}$

Tabelle 17: Vergleich der Ausbeuten von $\mathrm{Cp}^{*} \mathrm{TiF}_{3}, \mathrm{Cp}_{2} \mathrm{ZrF}_{2}$ und $\mathrm{Cp}_{2} \mathrm{HfF}_{2}$ unter Verwendung der Fluorierungsmittel 13 und 14 


\subsubsection{Darstellung von $\mathrm{Cp}^{*} \mathrm{Cp}^{\prime} \mathrm{HfCl}_{2}$ (16)}

$\mathrm{Cp}^{*} \mathrm{HfCl}_{3}(8.8 \mathrm{~g}, 21 \mathrm{mmol})$ wurde in THF $(100 \mathrm{ml})$ gelöst und bei $0{ }^{\circ} \mathrm{C}$ langsam mit einer Lösung von $\mathrm{LiC}_{5} \mathrm{H}_{4} \mathrm{SiMe}_{3}(3.0 \mathrm{~g}, 21 \mathrm{mmol})$ in THF $(50 \mathrm{ml})$ versetzt. Danach wurde das Lösungsmittel im Vakuum entfernt, der Rückstand in $n$-Hexan $(100 \mathrm{ml})$ aufgenommen und das $\mathrm{LiCl}$ abfiltriert. Das klare Filtrat wurde im Vakuum auf die Hälfte des Volumens eingeengt und über Nacht bei $-30{ }^{\circ} \mathrm{C}$ gelagert. Farblose Kristalle der Zielverbindung 16 wurden in einer Ausbeute von $2.7 \mathrm{~g}(25 \%)$ isoliert.

Schmelzpunkt: $201^{\circ} \mathrm{C}$.

Elementaranalyse für $\mathrm{C}_{18} \mathrm{H}_{28} \mathrm{Cl}_{2} \mathrm{HfSi}$ (521.91):

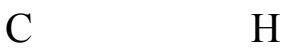

Ber. (\%): $\quad 41.42 \quad 5.41$,

Gef. $(\%): \quad \quad 41.6 \quad 5.3$.

${ }^{1} \mathrm{H}-\mathrm{NMR}-\mathrm{Spektrum}\left(200 \mathrm{MHz}, \mathrm{CDCl}_{3}\right): \delta 0.30\left(\mathrm{~s}, 9 \mathrm{H}, \mathrm{SiCH}_{3}\right), 2.08\left(\mathrm{~s}, 15 \mathrm{H}, \mathrm{C}_{5}\left(\mathrm{CH}_{3}\right)_{5}\right), 6.07$ $\left(\mathrm{t}, 2 \mathrm{H},{ }^{3} J_{\mathrm{H}-\mathrm{H}}=2.6 \mathrm{~Hz}, \mathrm{Cp}{ }^{\prime} H\right), 6.40\left(\mathrm{t}, 2 \mathrm{H},{ }^{3} J_{\mathrm{H}-\mathrm{H}}=2.6 \mathrm{~Hz}, \mathrm{Cp}^{\prime} H\right)$ ppm.

Massenspektrum (EI) m/z (\%): $\left[\mathrm{M}^{+}\right] 522(25),\left[\mathrm{M}^{+}-\mathrm{Me}\right] 507$ (100).

IR (Nujol, CsI): $\widetilde{v} 1630$ (s), 1406 (m), 1314 (m), 1244 (st), 1182 (m), 1075 (m) 1042 (m), 907 (st), 844 (sst), 820 (sst), 763 (st), 697 (m), 639 (m), 629 (m), 421 (m), 347 (m), 315 (st), $285(\mathrm{~s}) \mathrm{cm}^{-1}$. 


\subsubsection{Darstellung von $\mathrm{Cp}^{*} \mathrm{Cp}^{\prime} \mathrm{ZrF}_{2}(17)$}

Die Feststoffe Cp*Cp' $\mathrm{ZrCl}_{2}(0.50 \mathrm{~g}, 1.15 \mathrm{mmol})$ und 13 (0.46 g, $\left.1.15 \mathrm{mmol}\right)$ wurden miteinander vermengt. $\mathrm{CH}_{2} \mathrm{Cl}_{2}(20 \mathrm{ml})$ wurde einkondensiert und $24 \mathrm{~h}$ bei Raumtemperatur gerührt. Nach Abtrennung aller flüchtigen Bestandteile im Vakuum wurde der Rückstand in $n$-Hexan aufgenommen. Das Beiprodukt $\mathrm{Ph}_{2} \mathrm{PbCl}_{2}$ wurde abfiltriert und die Lösungsmittelmenge auf ca. $5 \mathrm{ml}$ reduziert. Nach Lagerung bei $-70{ }^{\circ} \mathrm{C}$ wurde 17 in Form farbloser Kristalle isoliert. Ausbeute: $0.39 \mathrm{~g}(85 \%)$.

Schmelzpunkt: $61{ }^{\circ} \mathrm{C}$.

Elementaranalyse für $\mathrm{C}_{18} \mathrm{H}_{28} \mathrm{~F}_{2} \mathrm{SiZr}$ (401.69):

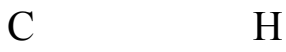

Ber. $(\%): \quad 53.82 \quad 7.00$,

Gef. $(\%)$ : $\quad 53.4 \quad 6.6$.

${ }^{1} \mathrm{H}-\mathrm{NMR}-$ Spektrum $\left(200 \mathrm{MHz}, \mathrm{CDCl}_{3}\right): \delta 0.21\left(\mathrm{~s}, 9 \mathrm{H}, \mathrm{SiCH}_{3}\right), 1.95\left(\mathrm{t},{ }^{4} J_{\mathrm{H}-\mathrm{F}}=0.8 \mathrm{~Hz}, 15 \mathrm{H}\right.$, $\left.\mathrm{C}_{5}\left(\mathrm{CH}_{3}\right)_{5}\right), 6.19$ - 6.24 (m, 2 H, Cp' $\left.H\right), 6.30$ - 6.35 (m, 2 H, Cp' $\left.H\right)$ ppm.

${ }^{19} \mathrm{~F}$ NMR-Spektrum (188 MHz, $\left.\mathrm{CDCl}_{3}\right): \delta 14.7\left(\mathrm{~s}, \mathrm{ZrF} \mathrm{F}_{2}\right)$.

Massenspektrum (EI) m/z (\%): $\left[\mathrm{M}^{+}\right] 401(25),\left[\mathrm{M}^{+}-\mathrm{Me}\right] 386(100)$.

IR (Nujol, CsI): $\widetilde{v} 1247$ (st), 1175 (m), 1051 (m), 1041 (s), 910 (m), 840 (sst), 809 (st), $756(\mathrm{~m}), 723$ (m), $632(\mathrm{~m}), 564(\mathrm{st}), 553(\mathrm{st}), 418(\mathrm{~m}), 378(\mathrm{st}), 355(\mathrm{~m}) \mathrm{cm}^{-1}$.

\subsubsection{Darstellung von $\mathrm{Cp}^{*} \mathrm{Cp}^{\prime} \mathrm{HfF}_{2}(18)$}

Nach Arbeitsvorschrift 4.3.17. wurde aus $\mathrm{Cp}^{*} \mathrm{Cp}^{\prime} \mathrm{HfCl}_{2}(0.5 \mathrm{~g}, 0.95 \mathrm{mmol})$ und $\mathbf{1 3}$ (0.38 g, $0.95 \mathrm{mmol}) 0.37 \mathrm{~g} \mathbf{1 8}(80 \%)$ isoliert. 
Schmelzpunkt: $68^{\circ} \mathrm{C}$.

Elementaranalyse für $\mathrm{C}_{18} \mathrm{H}_{28} \mathrm{~F}_{2} \mathrm{HfSi}$ (489.01):

$\begin{array}{lll} & \mathrm{C} & \mathrm{H} \\ \text { Ber. (\%): } & 44.21 & 5.77, \\ \text { Gef. (\%): } & 44.3 & 5.6 .\end{array}$

${ }^{1} \mathrm{H}-\mathrm{NMR}-$ Spektrum $\left(200 \mathrm{MHz}, \mathrm{CDCl}_{3}\right): \delta 0.21\left(\mathrm{~s}, 9 \mathrm{H}, \mathrm{SiCH}_{3}\right), 1.99\left(\mathrm{t},{ }^{4} J_{\mathrm{H}-\mathrm{F}}=0.6 \mathrm{~Hz}, 15 \mathrm{H}\right.$, $\left.\mathrm{C}_{5}\left(\mathrm{CH}_{3}\right)_{5}\right), 6.18$ - $6.22\left(\mathrm{~m}, 2 \mathrm{H}, \mathrm{Cp}^{\prime} H\right), 6.26$ - $6.31\left(\mathrm{~m}, 2 \mathrm{H}, \mathrm{Cp}{ }^{\prime} H\right) \mathrm{ppm}$.

${ }^{19} \mathrm{~F}$ NMR-Spektrum (188 MHz, $\left.\mathrm{CDCl}_{3}\right): \delta-22.6\left(\mathrm{~s}, \mathrm{Zr} F_{2}\right) \mathrm{ppm}$.

Massenspektrum (EI) m/z (\%): [M+] 489 (35), $\left[\mathrm{M}^{+}-\mathrm{Me}\right] 475$ (100).

IR (Nujol, CsI): $\widetilde{v} 1406$ (s), 1314 (s) 1249 (st), 1183 (m), 1070 (s), 1043 (m), 908 (m), 843 (sst), 812 (st), 758 (m), 724 (s), 697 (s), 643 (m), 630 (m); 559 (st), 537 (st), 451 (m), 351 (st), 300 (m), 224 (st), 220 (st) $\mathrm{cm}^{-1}$.

\subsubsection{Fluorierung von $\mathrm{Cp}^{*} \mathrm{TaCl}_{4}$ mit $\mathrm{Ph}_{3} \mathrm{BiF}_{2}$}

In einem NMR-Röhrchen wurden die Feststoffe $\mathrm{Cp}^{*} \mathrm{TaCl}_{4}(25 \mathrm{mg}, 0.05 \mathrm{mmol})$ und 14 (60 mg, $0.12 \mathrm{mmol})$ zusammengegeben und mit $\mathrm{C}_{6} \mathrm{D}_{6}(0.5 \mathrm{ml})$ überschichtet. Innerhalb von $1 \mathrm{~h}$ löste sich das $\mathrm{Cp}^{*} \mathrm{TaCl}_{4}$ unter Bildung einer schwach gelben Lösung auf. Nach $24 \mathrm{~h}$ wurden ${ }^{1} \mathrm{H}$ - und ${ }^{19} \mathrm{~F}-\mathrm{NMR}$ Spektren gemessen.

${ }^{1} \mathrm{H}-\mathrm{NMR}-$ Spektrum $\left(200 \mathrm{MHz}, \mathrm{C}_{6} \mathrm{D}_{6}\right): \delta 2.11\left(\mathrm{~s}, 15 \mathrm{H}, \mathrm{C}_{5}\left(\mathrm{CH}_{3}\right)_{5}\right), 6.92-6.90(\mathrm{~m}, 3 \mathrm{H}$, p-PhH), 7.03 - $7.12(\mathrm{~m}, 6 \mathrm{H}, m-\mathrm{Ph} H), 8.27$ - $8.36(\mathrm{~m}, 6 \mathrm{H}, o-\mathrm{Ph} H) \mathrm{ppm}$.

${ }^{19}$ F-NMR-Spektrum $\left(188 \mathrm{MHz}, \mathrm{C}_{6} \mathrm{D}_{6}\right): \delta-83.3$ (quint, $\left.{ }^{2} J_{\mathrm{F}-\mathrm{F}}=86.9 \mathrm{~Hz}, 1 \mathrm{~F}, F_{(\text {verb.) }}\right), 26.6[\mathrm{~d}$, $\left.{ }^{2} J_{\mathrm{F}-\mathrm{F}}=86.8 \mathrm{~Hz}, 4 \mathrm{~F}, F_{\text {(endst.) }}\right)$ ppm. 


\subsubsection{Darstellung von $\mu_{3}$-Fluoro- $\mu_{3}$-oxo-tris $(\mu$-isopropoxi)-hexakis(isopropoxi)-tri- $\operatorname{titan}(I V)(20)$}

Eine Suspension von $\mathrm{KHF}_{2}(0.5 \mathrm{~g}, 6.4 \mathrm{mmol})$ in Toluol $(50 \mathrm{ml})$ wurde mit $\mathrm{Ti}(\mathrm{O} i \mathrm{Pr})_{4}$ (4.0 ml, 3.8 g, $13.4 \mathrm{mmol})$ bei Raumtemperatur versetzt. Nach 4 h Refluxieren ließ sich eine Verkleinerung der Teilchengröße des Bodensatzes beobachten. Nach Filtration durch Celite wurden alle flüchtigen Bestandteile im Vakuum abgetrennt. Aus einem öligen, farblosen Rohprodukt wurde nach Umkristallisation aus Ether $(5 \mathrm{ml}) 2.1 \mathrm{~g}$ der Titelverbindung (65\%) in Form von farblosen Kristallen isoliert.

Schmelzpunkt: $89^{\circ} \mathrm{C}$.

Elementaranalyse für $\mathrm{C}_{27} \mathrm{H}_{63} \mathrm{FO}_{10} \mathrm{Ti}_{3}(710.47)$ :

$\begin{array}{llll} & \mathrm{C} & \mathrm{H} & \mathrm{F} \\ \text { Ber. (\%): } & 45.62 & 8.94 & 2.67, \\ \text { Gef. (\%): } & 45.2 & 8.8 & 2.9 .\end{array}$

${ }^{1} \mathrm{H}-\mathrm{NMR}-$ Spektrum $\left(200 \mathrm{MHz}, \mathrm{CDCl}_{3}\right): \delta 1.27$ - $1.49\left(\mathrm{~m}, 54 \mathrm{H}, \mathrm{OCH}\left(\mathrm{CH}_{3}\right)_{2}\right), 4.51-4.89(\mathrm{~m}$, $9 \mathrm{H}, \mathrm{OCH}) \mathrm{ppm}$.

${ }^{19}$ F-NMR-Spektrum $\left(188 \mathrm{MHz}, \mathrm{CDCl}_{3}\right): \delta-130.2\left(\mathrm{~s}, \mathrm{~F}, \mathrm{Ti}\left(\mu_{3}-F\right)\right),-122.4(\mathrm{~s}, \mathrm{br}, \mathrm{F}, \mathrm{Ti}(\mu F))$, 110.4 (s, br, F, Ti $\left.F_{(\text {endst.) }}\right)$ ppm.

Massenspektrum (EI) m/z (\%): [Ti(Oi-Pr $\left.)_{4}-\mathrm{Me}^{+}\right] 269$ (100), [Ti(Oi-Pr) $\left.{ }_{3}^{+}\right] 225$ (40).

IR (Nujol, NaCl): $\widetilde{v} 2620$ (m), 1615 (m), 1327 (st), 1261 (m), 1164 (st), 1128 (st), 1007 (st), 960 (sst), 855 (m), 804 (m), 643 (m), 592 (st) $\mathrm{cm}^{-1}$. 


\subsubsection{Reaktion von $\mathrm{Zr}(\mathrm{O} t-\mathrm{Bu})_{4}$ mit $\mathrm{KHF}_{2}$}

$\mathrm{KHF}_{2}(0.15 \mathrm{~g}, 2.0 \mathrm{mmol})$ wurde in Toluol $(20 \mathrm{ml})$ suspendiert und mit $\mathrm{Zr}(\mathrm{O} t-\mathrm{Bu})_{4}$ $(0.75 \mathrm{~g}, 2.0 \mathrm{mmol})$ versetzt. Nach $6 \mathrm{~h}$ Refluxieren wurde über Celite filtriert. Das Toluol wurde vollständig im Vakuum abgetrennt und der farblose, ölige Rückstand mit Ether (5 ml) versetzt. Nach einer Kristallisation bei $-30^{\circ} \mathrm{C}$ konnte ein farbloses Produkt isoliert werden (60 mg).

Schmelzpunkt: $168^{\circ} \mathrm{C}$.

${ }^{1} \mathrm{H}-\mathrm{NMR}$-Spektrum $\left(200 \mathrm{MHz}, \mathrm{CDCl}_{3}\right): \delta 1.30\left(\mathrm{~s}, 27 \mathrm{H}, \mathrm{OC}\left(\mathrm{CH}_{3}\right)_{3 \text { (endst.) }}\right), 1.40(\mathrm{~s}, 27 \mathrm{H}$, $\left.\mathrm{OC}\left(\mathrm{CH}_{3}\right)_{3 \text { (endst.) }}\right), 1.58\left(\mathrm{~s}, 9 \mathrm{H}, \mathrm{OC}\left(\mathrm{CH}_{3}\right)_{3 \text { (Anion) })}, 1.60\left(\mathrm{~s}, 27 \mathrm{H}, \mu \mathrm{OC}\left(\mathrm{CH}_{3}\right)_{3}\right) \mathrm{ppm}\right.$.

${ }^{19}$ F-NMR-Spektrum (188 MHz, $\mathrm{CDCl}_{3}$ ): kein Signal

FAB-MS (positiv) (NBA-Matrix): m/z (\%): [(Ot-Bu $\left.{ }_{6}(\mu \mathrm{O} t-\mathrm{Bu})_{3}\left(\mu_{3}-\mathrm{O}\right) \mathrm{Zr}_{3}{ }^{+}\right] 947(100)$.

IR (Nujol, KBr): $\widetilde{v} 1364(\mathrm{~m}), 1359(\mathrm{~m}), 1251$ (s), 1228 (s), 1198 (st), 1181 (st), 1022(m), 1011 (m), 978 (sst), 905 (m), 780 (m), 561 (m), 537 (m), 496 (m), 473 (m) cm . $^{-1}$.

\subsubsection{Darstellung von $\left[n-\mathrm{Bu}_{4} \mathrm{~N}\right]^{+}\left[(t-\mathrm{BuO})_{2} \mathrm{ZrF}_{3}\right]^{-}(22)$}

$\mathrm{Zu}$ einer Lösung von $\mathrm{Zr}(\mathrm{O} t-\mathrm{Bu})_{4}(0.75 \mathrm{~g}, 2.0 \mathrm{mmol})$ in $\mathrm{CH}_{2} \mathrm{Cl}_{2}(10 \mathrm{ml})$ wurde $\left[n-\mathrm{Bu}_{4} \mathrm{~N}\right]^{+}\left[\mathrm{HF}_{2}\right]^{-}\left(1.0 \mathrm{ml}, 2.0 \mathrm{mmol}, 50 \%\right.$ in $\left.\mathrm{CH}_{2} \mathrm{Cl}_{2}\right)$ zugespritzt. Nach $12 \mathrm{~h}$ Rühren wurden $5 \mathrm{ml}$ Lösungsmittel im Vakuum abgetrennt. Nach Kristallisation aus $\mathrm{CH}_{2} \mathrm{Cl}_{2}$ bei $-30{ }^{\circ} \mathrm{C}$ konnten $0.3 \mathrm{~g}$ der Titelverbindung isoliert werden.

Schmelzpunkt: $141^{\circ} \mathrm{C}$. 
Elementaranalyse für $\mathrm{C}_{24} \mathrm{H}_{54} \mathrm{NO}_{2} \mathrm{ZrF}_{3}(536.91)$ :

C

$\mathrm{H}$

Ber. (\%):

53.69

10.14,

Gef. (\%)

53.7

10.3 .

${ }^{1} \mathrm{H}-\mathrm{NMR}-$ Spektrum $\left(200 \mathrm{MHz}, \mathrm{CDCl}_{3}\right): \delta 0.91\left(\mathrm{t},{ }^{3} J_{\mathrm{H}-\mathrm{H}}=7 \mathrm{~Hz}, 12 \mathrm{H}, \mathrm{CH}_{2} \mathrm{CH}_{2} \mathrm{CH}_{2} \mathrm{CH}_{3}\right), 1.26$ (s, $18 \mathrm{H}, \mathrm{OC}\left(\mathrm{CH}_{3}\right)_{3}$ (endst.) $), 1.32-1.44\left(\mathrm{~m}, 8 \mathrm{H}, \mathrm{CH}_{2} \mathrm{CH}_{2} \mathrm{CH}_{2} \mathrm{CH}_{3}\right), 1.50$ - 1.67 (m, $8 \mathrm{H}$, $\left.\mathrm{CH}_{2} \mathrm{CH}_{2} \mathrm{CH}_{2} \mathrm{CH}_{3}\right), 3.21-3.34\left(\mathrm{~m}, 8 \mathrm{H}, \mathrm{CH}_{2} \mathrm{CH}_{2} \mathrm{CH}_{2} \mathrm{CH}_{3}\right)$ ppm.

${ }^{19}$ F-NMR-Spektrum (188 MHz, $\left.\mathrm{CDCl}_{3}\right): \delta-11.8\left(\mathrm{~s}, \mathrm{Zr} F_{(\text {endst. }}\right)$ ppm.

FAB-MS (positiv) (NBA-Matrix): m/z (\%): $\left[n-\mathrm{Bu}_{4} \mathrm{~N}\right] 242(100)$.

FAB-MS (negativ) (NBA-Matrix): m/z (\%): [(t-BuO) $\left.)_{2} \mathrm{ZrF}_{3} \times \mathrm{NBA}\right] 446(100)$.

IR (Nujol, KBr): $\widetilde{v} 1577(\mathrm{~m}), 1261$ (m), $1154(\mathrm{~s}), 1092(\mathrm{~m}), 1058$ (st), 1028 (st), 929 (m), $890(\mathrm{~m}), 801(\mathrm{~m}), 723(\mathrm{~m}), 486(\mathrm{sst}) \mathrm{cm}^{-1}$.

\subsubsection{Darstellung von $\operatorname{Bis}\left(\mu_{3}-0 x 0\right)$-hexakis $(\mu$-ethoxi)-octakis $(\mu$-oxo)- tetradekakis(ethoxi)-octa-tantal(V) (23)}

$\mathrm{Zu}$ einer Lösung von $\mathrm{Ta}(\mathrm{OEt})_{5}(1.0 \mathrm{ml}, 1.6 \mathrm{~g}, 3.9 \mathrm{mmol})$ in $\mathrm{CH}_{2} \mathrm{Cl}_{2}(30 \mathrm{ml})$ wurde eine Lösung von $\left[n-\mathrm{Bu}_{4} \mathrm{~N}\right]^{+}\left[\mathrm{HF}_{2}\right]^{-}\left(1.0 \mathrm{ml}, 2.0 \mathrm{mmol}, 50 \%\right.$ in $\left.\mathrm{CH}_{2} \mathrm{Cl}_{2}\right)$ bei Raumtemperatur zugespritzt. Nach 12 h Rühren wurden alle flüchtigen Bestandteile im Vakuum abgetrennt und der farblose, ölige Rückstand in Toluol $(10 \mathrm{ml})$ aufgenommen. Durch Kristallisation bei $-30{ }^{\circ} \mathrm{C}$ wurde die Titelverbindung in geringer Ausbeute isoliert.

Schmelzpunkt: $223^{\circ} \mathrm{C}$. 
Elementaranalyse für $\mathrm{C}_{40} \mathrm{H}_{100} \mathrm{O}_{30} \mathrm{Ta}_{8}$ (2508.21):

$\begin{array}{lll} & \mathrm{C} & \mathrm{H} \\ \text { Ber. (\%): } & 19.14 & 4.02, \\ \text { Gef. (\%): } & 19.3 & 4.1 .\end{array}$

${ }^{1} \mathrm{H}-\mathrm{NMR}-$ Spektrum $\left(200 \mathrm{MHz}, \mathrm{CDCl}_{3}\right): \delta 1.21-1.33\left(\mathrm{~m}, 60 \mathrm{H}, \mathrm{OCH}_{2} \mathrm{CH}_{3}\right), 4.32-4.84(\mathrm{~m}$, $\left.40 \mathrm{H}, \mathrm{OCH}_{2} \mathrm{CH}_{3}\right) \mathrm{ppm}$.

Massenspektrum (EI) m/z (\%): $\left[\mathrm{M}^{+}-\mathrm{OEt}\right] 2463$ (100), $\left[\mathrm{Ta}(\mathrm{OEt})_{4}{ }^{+}\right] 361(20)$.

IR (Nujol, KBr): $\widetilde{v} 1261$ (m), 1154 (m), 1114 (st), 1070 (st), 876 (m), 803 (m), 739 (sst), $511(\mathrm{st}) \mathrm{cm}^{-1}$.

\subsubsection{Darstellung von $\left[\left(n-\mathrm{Bu}_{4} \mathrm{~N}\right)^{+}\right]_{2}\left[\mathrm{SiF}_{6}\right]^{2-}(24)$}

$\mathrm{Zu}$ einer Lösung von $\mathrm{Si}(\mathrm{OEt})_{4}(0.75 \mathrm{~g}, 0.80 \mathrm{ml}, 3.59 \mathrm{mmol})$ in $n$-Hexan $(20 \mathrm{ml})$ wurde $\left[n-\mathrm{Bu}_{4} \mathrm{~N}\right]^{+}\left[\mathrm{HF}_{2}\right]^{-}\left(1.86 \mathrm{ml}, 3.59 \mathrm{mmol}, 50 \%\right.$ in $\left.\mathrm{CH}_{2} \mathrm{Cl}_{2}\right)$ zugespritzt. Das Lösungsmittel und überschüssiges $\mathrm{Si}(\mathrm{OEt})_{4}$ wurden im Vakuum abgetrennt und der ölige, farblose Rückstand in $\mathrm{CH}_{2} \mathrm{Cl}_{2}(10 \mathrm{ml})$ aufgenommen. Durch Kristallisation bei $-30{ }^{\circ} \mathrm{C}$ konnten $0.71 \mathrm{~g}$ der Titelverbindung isoliert werden.

Schmelzpunkt: $199^{\circ} \mathrm{C}$.

Elementaranalyse für $\mathrm{C}_{32} \mathrm{H}_{72} \mathrm{~F}_{6} \mathrm{~N}_{2} \mathrm{Si}(627.01)$ :

$\mathrm{C} \quad \mathrm{H}$

Ber. $(\%)$ : $\quad 61.30 \quad 11.57$,

Gef. (\%): $\quad 60.8 \quad 11.5$.

${ }^{1} \mathrm{H}-\mathrm{NMR}-$ Spektrum $\left(200 \mathrm{MHz}, \mathrm{CDCl}_{3}\right): \delta 0.91\left(\mathrm{t},{ }^{3} \mathrm{~J}_{\mathrm{H}-\mathrm{H}}=7 \mathrm{~Hz}, 36 \mathrm{H}, \mathrm{CH}_{2} \mathrm{CH}_{2} \mathrm{CH}_{2} \mathrm{CH}_{3}\right)$, $1.33-1.49\left(\mathrm{~m}, 24 \mathrm{H}, \mathrm{CH}_{2} \mathrm{CH}_{2} \mathrm{CH}_{2} \mathrm{CH}_{3}\right), \quad 1.50-1.67$ (m, 24 H, $\left.\mathrm{CH}_{2} \mathrm{CH}_{2} \mathrm{CH}_{2} \mathrm{CH}_{3}\right)$, 3.30 - $3.42\left(\mathrm{~m}, 24 \mathrm{H}, \mathrm{CH}_{2} \mathrm{CH}_{2} \mathrm{CH}_{2} \mathrm{CH}_{3}\right)$ ppm.

${ }^{19}$ F-NMR-Spektrum (188 MHz, $\left.\mathrm{CDCl}_{3}\right): \delta$-130.2 (s, $\left.\mathrm{Si} F_{6}\right)$ ppm. 
${ }^{29} \mathrm{Si}-\mathrm{NMR}-\mathrm{Spektrum}\left(124 \mathrm{MHz}, \mathrm{CDCl}_{3}\right): \delta$-186.7 (sept, $\left.{ }^{1} J_{\mathrm{Si}-\mathrm{F}}=113 \mathrm{~Hz}, \mathrm{SiF}_{6}\right) \mathrm{ppm}$.

FAB-MS (positiv) (NBA-Matrix): m/z (\%): $\left[n-\mathrm{Bu}_{4} \mathrm{~N}\right] 242$ (100).

FAB-MS (negativ) (NBA-Matrix): m/z (\%): $\left[\mathrm{SiF}_{5} \times \mathrm{NBA}\right] 276(100)$.

IR (Nujol, KBr): $\widetilde{v} 1262$ (s), 1153 (m), 1099 (m), 1059 (m), 1035 (m), 889 (m), 799 (m), 718 (sst), 702 (sst) 472 (st) $\mathrm{cm}^{-1}$.

\subsubsection{Alternative Darstellung von $\left[\mathrm{Bu}_{4} \mathrm{~N}\right]^{+}\left[\mathrm{Me}_{2} \mathrm{AlF}_{2}\right]^{-}$(25)}

$\mathrm{Zu}$ einer Lösung von $\left(\mathrm{Me}_{2} \mathrm{AlO} t-\mathrm{Bu}\right)_{2}(0.26 \mathrm{~g}, 1.0 \mathrm{mmol})$ in THF $(10 \mathrm{ml})$ wurde $\left[n-\mathrm{Bu}_{4} \mathrm{~N}\right]^{+}\left[\mathrm{HF}_{2}\right]^{-}\left(1.0 \mathrm{ml}, 2.0 \mathrm{mmol}, 50 \%\right.$ in $\left.\mathrm{CH}_{2} \mathrm{Cl}_{2}\right)$ bei Raumtemperatur zugespritzt. Es ließ sich eine spontane Gasentwicklung unter leichter Erwärmung beobachten. Nach $12 \mathrm{~h}$ Rühren bei Raumtemperatur wurde nach Umkristallisation aus $\mathrm{CH}_{2} \mathrm{Cl}_{2}$ bei $-30{ }^{\circ} \mathrm{C} 0.5 \mathrm{~g}(75 \%)$ der Titelverbindung isoliert.

Schmelzpunkt: $90{ }^{\circ} \mathrm{C}$

${ }^{1} \mathrm{H}-\mathrm{NMR}-$ Spektrum $\left(200 \mathrm{MHz}, \mathrm{CDCl}_{3}\right): \delta-1.15\left(\mathrm{t},{ }^{3} J_{\mathrm{F}-\mathrm{H}}=2.7 \mathrm{~Hz}, 6 \mathrm{H}, \mathrm{AlCH} H_{3}\right), 0.92$ (t, $\left.{ }^{3} J_{\mathrm{H}-\mathrm{H}}=7 \mathrm{~Hz}, 12 \mathrm{H}, \mathrm{CH}_{2} \mathrm{CH}_{2} \mathrm{CH}_{2} \mathrm{CH}_{3}\right), 1.28-1.46\left(\mathrm{~m}, 8 \mathrm{H}, \mathrm{CH}_{2} \mathrm{CH}_{2} \mathrm{CH}_{2} \mathrm{CH}_{3}\right), 1.49$ - 1.66 (m, $8 \mathrm{H}, \mathrm{CH}_{2} \mathrm{CH}_{2} \mathrm{CH}_{2} \mathrm{CH}_{3}$ ), 3.13 - 3.22 (m, $8 \mathrm{H}, \mathrm{CH}_{2} \mathrm{CH}_{2} \mathrm{CH}_{2} \mathrm{CH}_{3}$ ) ppm.

${ }^{19}$ F-NMR-Spektrum (188 MHz, $\left.\mathrm{CDCl}_{3}\right): \delta-155.7$ (s, br, $\left.\mathrm{Al} F_{2}\right)$ ppm.

${ }^{27}$ Al-NMR-Spektrum (65 MHz, $\left.\mathrm{CDCl}_{3}\right): \delta 127.0$ (s, br) ppm. 


\subsubsection{Darstellung von $\left[n-\mathrm{Bu}_{4} \mathrm{~N}\right]^{+}\left[\mathrm{VO}\left(\operatorname{acac}^{-} \mathrm{F}_{2}\right]^{-}(27)\right.$}

$\mathrm{VO}(\mathrm{acac})_{2}(0.51 \mathrm{~g}, 2.0 \mathrm{mmol})$ gelöst in THF $(10 \mathrm{ml})$ wurde mit $\left[n-\mathrm{Bu}_{4} \mathrm{~N}\right]^{+}\left[\mathrm{HF}_{2}\right]^{-}$ (1.0 ml, $2 \mathrm{mmol}, 50 \%$ in $\mathrm{CH}_{2} \mathrm{Cl}_{2}$ ) bei $0{ }^{\circ} \mathrm{C}$ versetzt. Dabei veränderten sich weder Farbe noch Temperatur. Nach zweistündigem Rühren bei Raumtemperatur und Entfernen des Lösungsmittel im Vakuum wurde ein tiefgrünes Ö1 isoliert.

${ }^{1} \mathrm{H}-\mathrm{NMR}-$ Spektrum $\left(200 \mathrm{MHz}, \mathrm{CDCl}_{3}\right): \delta 0.88$ (s, br, $\mathrm{CH}_{2} \mathrm{CH}_{2} \mathrm{CH}_{2} \mathrm{CH}_{3}$ ), 1.34 (s, br, $\mathrm{CH}_{2} \mathrm{CH}_{2} \mathrm{CH}_{2} \mathrm{CH}_{3}$ ), 2.15 (s, br, $\mathrm{C}(\mathrm{O}) \mathrm{CH}_{3}$ ), 3.52 (s, br, $\mathrm{CH}_{2} \mathrm{CH}_{2} \mathrm{CH}_{2} \mathrm{CH}_{3}$ ) ppm.

${ }^{19} \mathrm{~F}-\mathrm{NMR}-$ Spektrum $\left(188 \mathrm{MHz}, \mathrm{CDCl}_{3}\right): \delta-28.0\left(\mathrm{~s}, \mathrm{br}, \mathrm{VF}{ }_{2}\right) \mathrm{ppm}$.

FAB-MS (positiv) (NBA-Matrix): m/z (\%): [n-Bu $\left.\mathrm{Bu}_{4}\right] 242(100)$.

FAB-MS (negativ) (NBA-Matrix): m/z (\%): [VO(acac)F 204 (100).

\subsubsection{Darstellung von $\left[n-\mathrm{Bu}_{4} \mathrm{~N}\right]^{+}\left[\mathrm{O}_{2} \mathrm{Mo}(\operatorname{acac}) \mathrm{F}_{2}\right]^{-} \times 0.5$ THF (28)}

Der Vorschrift 2.3.26. folgend wurde $\mathrm{MoO}_{2}(\mathrm{acac})_{2} \quad(0.5 \mathrm{~g}, 1.5 \mathrm{mmol})$ mit $\left[n-\mathrm{Bu}_{4} \mathrm{~N}\right]^{+}\left[\mathrm{HF}_{2}\right]^{-}\left(0.75 \mathrm{ml}, 1.5 \mathrm{mmol}, 50 \%\right.$ in $\left.\mathrm{CH}_{2} \mathrm{Cl}_{2}\right)$ zu 28 umgesetzt, das als gelbes Ö1 anfiel.

${ }^{1} \mathrm{H}-\mathrm{NMR}-$ Spektrum $\left(200 \mathrm{MHz}, \mathrm{CDCl}_{3}\right): \delta 0.92\left(\mathrm{t},{ }^{3} \mathrm{~J}_{\mathrm{H}-\mathrm{H}}=7 \mathrm{~Hz}, 12 \mathrm{H}, \mathrm{CH}_{2} \mathrm{CH}_{2} \mathrm{CH}_{2} \mathrm{CH}_{3}\right)$, 1.28 - $1.43\left(\mathrm{~m}, 8 \mathrm{H}, \mathrm{CH}_{2} \mathrm{CH}_{2} \mathrm{CH}_{2} \mathrm{CH}_{3}\right), 1.49$ - $1.66\left(\mathrm{~m}, 8 \mathrm{H}, \mathrm{CH}_{2} \mathrm{CH}_{2} \mathrm{CH}_{2} \mathrm{CH}_{3}\right), 1.75$ - 1.82 (m, $1 \mathrm{H}, \mathrm{THF}), 1.96\left(\mathrm{~s}, 6 \mathrm{H}, \mathrm{C}(\mathrm{O}) \mathrm{CH}_{3}\right), 3.13$ - $3.28\left(\mathrm{~m}, 8 \mathrm{H}, \mathrm{CH}_{2} \mathrm{CH}_{2} \mathrm{CH}_{2} \mathrm{CH}_{3}\right), 3.66$ - 3.72 (m, 1 H, THF) ppm.

${ }^{19}$ F-NMR-Spektrum $\left(188 \mathrm{MHz}, \mathrm{CDCl}_{3}\right): \delta-57.8\left(\mathrm{~s}, \mathrm{Mo} F_{2}\right) \mathrm{ppm}$.

FAB-MS (positiv) (NBA-Matrix): m/z (\%): [n-Bu $\mathrm{Bu}_{4} 242$ (100).

FAB-MS (negativ) (NBA-Matrix): m/z (\%): [ $\left.\mathrm{MoO}_{2}(\mathrm{acac}) \mathrm{F}_{2}\right] 267$ (100). 


\subsection{Behandlung und Entsorgung der Abfälle}

Die eingesetzten Lösungsmittel wurden abdestilliert oder im Vakuum in Kühlfallen einkondensiert und getrennt nach halogenhaltigen bzw. halogenfreien Abfällen in die bereitgestellten Behälter gegeben. Aceton aus Kühlbädern und der Reinigung der Glasgeräte wurde destillativ aufgereinigt und erneut eingesetzt. Die deuterierten Lösungsmittel aus den NMR-Messungen wurden zur späteren Destillation in getrennten Gefäßen gesammelt. Natriumrückstände wurden in Ethanol aufgelöst und danach in das $\mathrm{KOH}-$ Reinigungsbad für Glasgeräte gegeben. Die beim Vortrocknen der Lösungsmittel angefallenen festen $\mathrm{KOH}-$ Rückstände wurden ebenfalls dem KOH-Reinigungsbad zugeführt. Andere Trockenmittelreste wie Calciumdihydrid oder $\mathrm{P}_{4} \mathrm{O}_{10}$ überführte man nach vorsichtiger, vollständiger Hydrolyse mit Wasser in die Basen- bzw. Säureabfälle. Konzentrierte Salpetersäure und Königswasser zur Reinigung von Glasfiltern wurden in der Regel mehrmals verwendet, danach neutralisiert und in den Schwermetallabfallbehältern gesammelt. Das bei den Fluorierungen anfallende Trimethylzinnchlorid wurde getrennt gesammelt und mit Natriumflourid regeneriert. Schwermetallhaltige Abfälle wurden in den dafür vorgesehenen Behälter entsorgt. Alle anderen Chemikalienrückstände wurden vorsichtig mit wäßrigem Aceton hydrolysiert und je nach Zusammensetzung in die Gefäße für saure bzw. basische Abfälle gegeben. Die in die bereitgestellten Kanister entsorgten Abfälle wurden in den ausliegenden Listen ordnungsgemäß deklariert.

Die bei Anfertigung dieser Arbeit angefallenen Mengen Sonderabfall können wie folgt abgeschätzt werden:

Halogenhaltige Lösungsmittelabfälle

Halogenfreie Lösungsmittelabfälle

Schwermetallhaltige Abfälle

Säureabfälle

Basenabfälle
101

101

$1000 \mathrm{~g}$

201

201 


\section{Kristallographischer Teil}

Verbindung

Archivnummer

Summenformel

Molmasse

Temperatur

Wellenlänge

Kristallsystem

Raumgruppe

Zelldimensionen

Zellvolumen

Formeleinheiten pro Zelle $Z$

Berechnete Dichte

Absorptionskoeffizient

$F(000)$

Kristallgröße

Gemessener $\theta$-Bereich

Indexgrenzen

Anzahl gemessener Reflexe

Unabhängige Reflexe

Daten/Restraints/Parameter

Goodness-of-Fit an $F^{2}$

Endgültige $R$-Werte [I $>2 \sigma(\mathrm{I})]$

$R$-Werte (sämtliche Daten)

Extinktionskoeffizient

Größtes Maximum und Minimum
1

CCDC 91721

$\mathrm{C}_{7} \mathrm{H}_{19} \mathrm{Cl}_{4} \mathrm{Si}_{2} \mathrm{Ta}$

482.15

203.(2) K

$71.073 \mathrm{pm}$

Triklin

$P \overline{1}$

$a=904.6(2) \mathrm{pm} ; \quad \alpha=66.54(3)^{\circ}$

$b=1347.7(3) \mathrm{pm} ; \quad \beta=82.71(3)^{\circ}$

$c=1458.1(3) \mathrm{pm} ; \quad \gamma=79.36(3)^{\circ}$

$1.5998(5) \mathrm{nm}^{3}$

4

$2.002 \mathrm{Mg} / \mathrm{m}^{3}$

$7.657 \mathrm{~mm}^{-1}$

920

$0.80 \times 0.80 \times 0.60$

3.55 bis $22.47^{\circ}$

$-9 \leq h \leq 9,-11 \leq k \leq 14,-15 \leq l \leq 15$

5287

$4120\left(R_{\text {int }}=0.0725\right)$

4115 / 0 / 266

1.125

$R 1=0.0435, w R 2=0.1116$

$R 1=0.0445, w R 2=0.1168$

$0.0019(3)$

3471 und -2535 e $\times \mathrm{nm}^{-3}$ 
Verbindung

Archivnummer

Summenformel

Molmasse

Temperatur

Wellenlänge

Kristallsystem

Raumgruppe

Zelldimensionen

Zellvolumen

Formeleinheiten pro Zelle $Z$

Berechnete Dichte

Absorptionskoeffizient

$F(000)$

Kristallgröße

Gemessener $\theta$-Bereich

Indexgrenzen

Anzahl gemessener Reflexe

Unabhängige Reflexe

Daten/Restraints/Parameter

Goodness-of-Fit an $F^{2}$

Endgültige $R$-Werte [I $>2 \sigma(\mathrm{I})]$

$R$-Werte (sämtliche Daten)

Extinktionskoeffizient

Größtes Maximum und Minimum

\section{3}

CCDC 91724

$\mathrm{C}_{7} \mathrm{H}_{19} \mathrm{Cl}_{4} \mathrm{Si}_{2} \mathrm{Ta}+0.5 \mathrm{C}_{14} \mathrm{H}_{38} \mathrm{Cl}_{6} \mathrm{OSi}_{4} \mathrm{Ta}_{2}$

$482.15+0.5 \times 909.41$

133.(2) K

$71.073 \mathrm{pm}$

Triklin

$P \overline{1}$

$a=899.0(3) \mathrm{pm} ; \quad \alpha=89.77(3)^{\circ}$

$b=1337.0(3) \mathrm{pm} ; \quad \beta=76.56(3)^{\circ}$

$c=1366.8(3) \mathrm{pm} ; \quad \gamma=79.18(3)^{\circ}$

$1.5681(7) \mathrm{nm}^{3}$

2

$1.984 \mathrm{Mg} / \mathrm{m}^{3}$

$7.727 \mathrm{~mm}^{-1}$

894

$0.5 \times 0.4 \times 0.4 \mathrm{~mm}^{3}$

2.14 bis $25.00^{\circ}$

$-10 \leq h \leq 10,-15 \leq k \leq 15,0 \leq l \leq 16$

5513

$4814\left(R_{\text {int }}=0.00204\right)$

$5513 / 0 / 263$

1.101

$R 1=0.0275, w R 2=0.0654$

$R 1=0.0333, w R 2=0.0667$

0.00204(14)

1675 und $-1451 \mathrm{e} \times \mathrm{nm}^{-3}$ 
Verbindung

Archivnummer

Summenformel

Molmasse

Temperatur

Wellenlänge

Kristallsystem

Raumgruppe

Zelldimensionen

Zellvolumen

Formeleinheiten pro Zelle $Z$

Berechnete Dichte

Absorptionskoeffizient

$F(000)$

Kristallgröße

Gemessener $\theta$-Bereich

Indexgrenzen

Anzahl gemessener Reflexe

Unabhängige Reflexe

Daten/Restraints/Parameter

Goodness-of-Fit an $F^{2}$

Endgültige $R$-Werte [I $>2 \sigma(\mathrm{I})$ ]

$R$-Werte (sämtliche Daten)

Größtes Maximum und Minimum

\section{4}

CCDC 147049

$\mathrm{C}_{7} \mathrm{H}_{19} \mathrm{Cl}_{4} \mathrm{NbSi}_{2}$

394.11

200.(2) K

$71.073 \mathrm{pm}$

Monoklin

$P 2{ }_{1} / c$

$a=1536.2(3) \mathrm{pm} ; \quad \alpha=90.00^{\circ}$

$b=915.9(2) \mathrm{pm} ; \quad \beta=106.42(3)^{\circ}$

$c=1211.6(2) \mathrm{pm} ; \quad \gamma=90.00^{\circ}$

$1.6351(6) \mathrm{nm}^{3}$

4

$1.601 \mathrm{Mg} / \mathrm{m}^{3}$

$1.506 \mathrm{~mm}^{-1}$

792

$0.9 \times 0.4 \times 0.4 \mathrm{~mm}^{3}$

3.51 bis $24.94^{\circ}$

$-15 \leq h \leq 18,-10 \leq k \leq 6,-14 \leq l \leq 14$

3028

$2842\left(R_{\text {int }}=0.0520\right)$

$2837 / 0 / 133$

1.052

$R 1=0.0531, w R 2=0.1327$

$R 1=0.0562, w R 2=0.1427$

4719 und $-716 \mathrm{e} \times \mathrm{nm}^{-3}$ 
Verbindung

Archivnummer

Summenformel

Molmasse

Temperatur

Wellenlänge

Kristallsystem

Raumgruppe

Zelldimensionen

Zellvolumen

Formeleinheiten pro Zelle $Z$

Berechnete Dichte

Absorptionskoeffizient

$F(000)$

Kristallgröße

Gemessener $\theta$-Bereich

Indexgrenzen

Anzahl gemessener Reflexe

Unabhängige Reflexe

Daten/Restraints/Parameter

Goodness-of-Fit an $F^{2}$

Endgültige R-Werte [I > $2 \sigma(\mathrm{I})]$

$R$-Werte (sämtliche Daten)

Größtes Maximum und Minimum

\section{8}

roe733

$\mathrm{C}_{12} \mathrm{H}_{33} \mathrm{ClSi}_{3} \mathrm{Sn}$

415.06

200.(2) K

$71.073 \mathrm{pm}$

Hexagonal

$P 6_{3} / m$

$a=1057.89(7) \mathrm{pm} ; \quad \alpha=90.00^{\circ}$

$b=1057.89(7) \mathrm{pm} ; \quad \beta=90.00^{\circ}$

$c=1101.2(2) \mathrm{pm} ; \quad \gamma=120.00^{\circ}$

$1.0673(2) \mathrm{nm}^{3}$

3

$2.294 \mathrm{Mg} / \mathrm{m}^{3}$

$1.476 \mathrm{~mm}^{-1}$

428

$0.6 \times 0.2 \times 0.2 \mathrm{~mm}^{3}$

3.70 bis $24.95^{\circ}$

$-10 \leq h \leq 10,-12 \leq k \leq 12,-12 \leq l \leq 12$

1982

$661\left(R_{\text {int }}=0.0661\right)$

$661 / 0 / 34$

1.270

$R 1=0.0415, w R 2=0.1038$

$R 1=0.0438, w R 2=0.1048$

1308 und $-2192 \mathrm{e} \times \mathrm{nm}^{-3}$ 
Verbindung

Archivnummer

Summenformel

Molmasse

Temperatur

Wellenlänge

Kristallsystem

Raumgruppe

Zelldimensionen

Zellvolumen

Formeleinheiten pro Zelle $Z$

Berechnete Dichte

Absorptionskoeffizient

$F(000)$

Kristallgröße

Gemessener $\theta$-Bereich

Indexgrenzen

Anzahl gemessener Reflexe

Unabhängige Reflexe

Daten/Restraints/Parameter

Goodness-of-Fit an $F^{2}$

Endgültige $R$-Werte [I $>2 \sigma(\mathrm{I})$ ]

$R$-Werte (sämtliche Daten)

Extinktionskoeffizient

Größtes Maximum und Minimum
9

CCDC 91725

$\mathrm{C}_{14} \mathrm{H}_{38} \mathrm{~F}_{8} \mathrm{Si}_{4} \mathrm{Ta}_{2}$

832.70

203.(2) K

$71.073 \mathrm{pm}$

Monoklin

$P 2{ }_{1} / c$

$a=638.58(13) \mathrm{pm} ; \alpha=90.00^{\circ}$

$b=2493.5(5) \mathrm{pm} ; \quad \beta=93.63(3)^{\circ}$

$c=1740.6(4) \mathrm{pm} ; \quad \gamma=90.00^{\circ}$

$2.7660(10) \mathrm{nm}^{3}$

4

$2.000 \mathrm{Mg} / \mathrm{m}^{3}$

$8.133 \mathrm{~mm}^{-1}$

1584

$0.80 \times 0.30 \times 0.30$

3.57 bis $22.46^{\circ}$

$-6 \leq h \leq 6,0 \leq k \leq 26,-18 \leq l \leq 18$

3587

$3544\left(R_{\text {int }}=0.1115\right)$

3534 / 0 / 266

1.233

$R 1=0.0526, w R 2=0.1485$

$R 1=0.0561, w R 2=0.1534$

$0.00095(13)$

2969 und $-1342 \mathrm{e} \times \mathrm{nm}^{-3}$ 
Verbindung

Archivnummer

Summenformel

Molmasse

Temperatur

Wellenlänge

Kristallsystem

Raumgruppe

Zelldimensionen

Zellvolumen

Formeleinheiten pro Zelle $Z$

Berechnete Dichte

Absorptionskoeffizient

$F(000)$

Kristallgröße

Gemessener $\theta$-Bereich

Indexgrenzen

Anzahl gemessener Reflexe

Unabhängige Reflexe

Daten/Restraints/Parameter

Goodness-of-Fit an $F^{2}$

Endgültige $R$-Werte [I $>2 \sigma(\mathrm{I})]$

$R$-Werte (sämtliche Daten)

Größtes Maximum und Minimum
12

roe 831

$\mathrm{C}_{51} \mathrm{H}_{115} \mathrm{Na}_{4} \mathrm{O}_{4} \mathrm{Si}_{4}$ incl. $n$-Hexan

996.75

203.(2) K

$71.073 \mathrm{pm}$

Monoklin

$P 2{ }_{1} / n$

$a=1300.25(12) \mathrm{pm} ; \alpha=90.00^{\circ}$

$b=2225.3(4) \mathrm{pm} ; \quad \beta=95.086(10)^{\circ}$

$c=2187.5(4) \mathrm{pm} ; \quad \gamma=90.00^{\circ}$

$6.305(2) \mathrm{nm}^{3}$

4

$1.050 \mathrm{Mg} / \mathrm{m}^{3}$

$0.158 \mathrm{~mm}^{-1}$

2212

$1.0 \times 0.8 \times 0.6 \mathrm{~mm}^{3}$

3.51 bis $22.54^{\circ}$

$-14 \leq h \leq 14,-7 \leq k \leq 23,-23 \leq l \leq 23$

11761

$8232\left(R_{\text {int }}=0.0264\right)$

$8228 / 0$ / 605

1.021

$R 1=0.0418, w R 2=0.1074$

$R 1=0.0510, w R 2=0.1175$

303 und $-240 \mathrm{e} \times \mathrm{nm}^{-3}$ 
Verbindung

Archivnummer

Summenformel

Molmasse

Temperatur

Wellenlänge

Kristallsystem

Raumgruppe

Zelldimensionen

Zellvolumen

Formeleinheiten pro Zelle $Z$

Berechnete Dichte

Absorptionskoeffizient

$F(000)$

Kristallgröße

Gemessener $\theta$-Bereich

Indexgrenzen

Anzahl gemessener Reflexe

Unabhängige Reflexe

Daten/Restraints/Parameter

Goodness-of-Fit an $F^{2}$

Endgültige $R$-Werte [I $>2 \sigma(\mathrm{I})$ ]

$R$-Werte (sämtliche Daten)

Extinktionskoeffizient

Größtes Maximum und Minimum
14

roe 775

$\mathrm{C}_{18} \mathrm{H}_{15} \mathrm{BiF}_{2}$

478.28

293.(2) K

$71.073 \mathrm{pm}$

Monoklin

$C 2 / c$

$a=2594.91(5) \mathrm{pm} ; \quad \alpha=90.00^{\circ}$

$b=905.8(2) \mathrm{pm} ; \quad \beta=129.86(3)^{\circ}$

$c=1796.4(4) \mathrm{pm} ; \quad \gamma=90.00^{\circ}$

$3.183(11) \mathrm{nm}^{3}$

8

$1.996 \mathrm{Mg} / \mathrm{m}^{3}$

$11.086 \mathrm{~mm}^{-1}$

1792

$0.8 \times 0.4 \times 0.3 \mathrm{~mm}^{3}$

3.85 bis $22.48^{\circ}$

$-24 \leq h \leq 27,-9 \leq k \leq 9,-19 \leq l \leq 19$

3537

$2068\left(R_{\text {int }}=0.0889\right)$

2068 / 0 / 191

1.028

$R 1=0.1047, w R 2=0.2505$

$R 1=0.1398, w R 2=0.2991$

$0.0000(2)$

450 und $-531 \mathrm{e} \times \mathrm{nm}^{-3}$ 
Verbindung

Archivnummer

Summenformel

Molmasse

Temperatur

Wellenlänge

Kristallsystem

Raumgruppe

Zelldimensionen

Zellvolumen

Formeleinheiten pro Zelle $Z$

Berechnete Dichte

Absorptionskoeffizient

$F(000)$

Kristallgröße

Gemessener $\theta$-Bereich

Indexgrenzen

Anzahl gemessener Reflexe

Unabhängige Reflexe

Daten/Restraints/Parameter

Goodness-of-Fit an $F^{2}$

Endgültige $R$-Werte [I $>2 \sigma(\mathrm{I})$ ]

$R$-Werte (sämtliche Daten)

Größtes Maximum und Minimum
15

CCDC 128543

$\mathrm{C}_{18} \mathrm{H}_{28} \mathrm{Cl}_{2} \mathrm{SiZr}$

434.61

153.(2) K

$71.073 \mathrm{pm}$

Orthorhombisch

$P 2{ }_{1}{ }_{1} 2_{1}$

$a=1194.41(13) \mathrm{pm} ; \alpha=90.00^{\circ}$

$b=1280.0(2) \mathrm{pm} ; \quad \beta=90.00^{\circ}$

$c=1294.0(2) \mathrm{pm} ; \quad \gamma=90.00^{\circ}$

$1.9784(5) \mathrm{nm}^{3}$

4

$1.459 \mathrm{Mg} / \mathrm{m}^{3}$

$0.882 \mathrm{~mm}^{-1}$

896

$0.9 \times 0.5 \times 0.3 \mathrm{~mm}^{3}$

3.53 bis $25.02^{\circ}$

$-5 \leq h \leq 14,-15 \leq k \leq 15,-15 \leq l \leq 15$

3091

$2779\left(R_{\text {int }}=0.0736\right)$

2777 / 0 / 207

1.033

$R 1=0.0501, w R 2=0.1280$

$R 1=0.0515, w R 2=0.1308$

971 und $-1394 \mathrm{e} \times \mathrm{nm}^{-3}$ 
Verbindung

Archivnummer

Summenformel

Molmasse

Temperatur

Wellenlänge

Kristallsystem

Raumgruppe

Zelldimensionen

Zellvolumen

Formeleinheiten pro Zelle $Z$

Berechnete Dichte

Absorptionskoeffizient

$F(000)$

Kristallgröße

Gemessener $\theta$-Bereich

Indexgrenzen

Anzahl gemessener Reflexe

Unabhängige Reflexe

Daten/Restraints/Parameter

Goodness-of-Fit an $F^{2}$

Endgültige $R$-Werte [I $>2 \sigma(\mathrm{I})$ ]

$R$-Werte (sämtliche Daten)

Größtes Maximum und Minimum
17

CCDC 128544

$\mathrm{C}_{18} \mathrm{H}_{28} \mathrm{~F}_{2} \mathrm{SiZr}$

401.71

153.(2) K

$71.073 \mathrm{pm}$

Monoklin

$C 2 / c$

$a=3658 .(3) \mathrm{pm} ; \quad \alpha=90.00^{\circ}$

$b=852.3(2) \mathrm{pm} ; \quad \beta=102.66(3)^{\circ}$

$c=1238.7(3) \mathrm{pm} ; \quad \gamma=90.00^{\circ}$

$3.768(3) \mathrm{nm}^{3}$

8

$1.416 \mathrm{Mg} / \mathrm{m}^{3}$

$0.660 \mathrm{~mm}^{-1}$

1664

$1.0 \times 0.6 \times 0.3 \mathrm{~mm}^{3}$

3.57 bis $22.52^{\circ}$

$-39 \leq h \leq 38,-9 \leq k \leq 9,-1 \leq l \leq 13$

2654

$2464\left(R_{\text {int }}=0.0412\right)$

$2461 / 0 / 207$

1.027

$R 1=0.0322, w R 2=0.0826$

$R 1=0.0351, w R 2=0.0872$

473 und $-477 \mathrm{e} \times \mathrm{nm}^{-3}$ 
Verbindung

Archivnummer

Summenformel

Molmasse

Temperatur

Wellenlänge

Kristallsystem

Raumgruppe

Zelldimensionen

Zellvolumen

Formeleinheiten pro Zelle $Z$

Berechnete Dichte

Absorptionskoeffizient

$F(000)$

Kristallgröße

Gemessener $\theta$-Bereich

Indexgrenzen

Anzahl gemessener Reflexe

Unabhängige Reflexe

Daten/Restraints/Parameter

Goodness-of-Fit an $F^{2}$

Endgültige $R$-Werte $[\mathrm{I}>2 \sigma(\mathrm{I})]$

$R$-Werte (sämtliche Daten)

Größtes Maximum und Minimum
18

CCDC 128545

$\mathrm{C}_{18} \mathrm{H}_{28} \mathrm{Cl}_{2} \mathrm{HfSi}$

488.98

153.(2) K

$71.073 \mathrm{pm}$

Monoklin

$P 2{ }_{1} / c$

$a=1193.6(8) \mathrm{pm} ; \quad \alpha=90.00^{\circ}$

$b=1833 .(4) \mathrm{pm} ; \quad \beta=99.77(10)^{\circ}$

$c=896.7(14) \mathrm{pm} ; \quad \gamma=90.00^{\circ}$

$1.934(5) \mathrm{nm}^{3}$

4

$1.679 \mathrm{Mg} / \mathrm{m}^{3}$

$5.468 \mathrm{~mm}^{-1}$

960

$0.8 \times 0.8 \times 0.7 \mathrm{~mm}^{3}$

3.64 bis $22.50^{\circ}$

$-11 \leq h \leq 12,-19 \leq k \leq 0,-9 \leq l \leq 9$

2528

$2509\left(R_{\text {int }}=0.0870\right)$

2506 / 333 / 207

1.109

$R 1=0.0515, w R 2=0.1357$

$R 1=0.0537, w R 2=0.1433$

3069 und $-2957 \mathrm{e} \times \mathrm{nm}^{-3}$ 
Verbindung

Archivnummer

Summenformel

Molmasse

Temperatur

Wellenlänge

Kristallsystem

Raumgruppe

Zelldimensionen

Zellvolumen

Formeleinheiten pro Zelle $Z$

Berechnete Dichte

Absorptionskoeffizient

$F(000)$

Kristallgröße

Gemessener $\theta$-Bereich

Indexgrenzen

Anzahl gemessener Reflexe

Unabhängige Reflexe

Daten/Restraints/Parameter

Goodness-of-Fit an $F^{2}$

Endgültige $R$-Werte [I $>2 \sigma(\mathrm{I})]$

$R$-Werte (sämtliche Daten)

Extinktionskoeffizient

Größtes Maximum und Minimum
20

CCDC 147050

$\mathrm{C}_{27} \mathrm{H}_{63} \mathrm{FO}_{10} \mathrm{Ti}_{3}$

710.47

200.(2) K

$71.073 \mathrm{pm}$

Monoklin

$P 2{ }_{1} / n$

$a=1277.5(2) \mathrm{pm} ; \quad \alpha=90.00^{\circ}$

$b=1759.2(3) \mathrm{pm} ; \quad \beta=92.58(2)^{\circ}$

$c=1710.3(2) \mathrm{pm} ; \quad \gamma=90.00^{\circ}$

$3.8399(10) \mathrm{nm}^{3}$

4

$1.229 \mathrm{Mg} / \mathrm{m}^{3}$

$0.657 \mathrm{~mm}^{-1}$

1520

$1.0 \times 0.6 \times 0.6 \mathrm{~mm}^{3}$

3.55 bis $25.05^{\circ}$

$-15 \leq h \leq 15,-7 \leq k \leq 20,-20 \leq l \leq 20$

10063

$6766\left(R_{\text {int }}=0.0309\right)$

6760 / 0 / 389

1.048

$R 1=0.0656, w R 2=0.1532$

$R 1=0.0979, w R 2=0.1831$

$0.0001(3)$

745 und $-639 \mathrm{e} \times \mathrm{nm}^{-3}$ 
Verbindung

Archivnummer

Summenformel

Molmasse

Temperatur

Wellenlänge

Kristallsystem

Raumgruppe

Zelldimensionen

Zellvolumen

Formeleinheiten pro Zelle $Z$

Berechnete Dichte

Absorptionskoeffizient

$F(000)$

Kristallgröße

Gemessener $\theta$-Bereich

Indexgrenzen

Anzahl gemessener Reflexe

Unabhängige Reflexe

Daten/Restraints/Parameter

Goodness-of-Fit an $F^{2}$

Endgültige $R$-Werte $[\mathrm{I}>2 \sigma(\mathrm{I})]$

$R$-Werte (sämtliche Daten)

Größtes Maximum und Minimum
23

CCDC 147051

$\mathrm{C}_{40} \mathrm{H}_{100} \mathrm{O}_{30} \mathrm{Ta}_{8}$

2508.80

200.(2) K

$71.073 \mathrm{pm}$

Monoklin

$P 2{ }_{1} / n$

$a=1466.8(3) \mathrm{pm} ; \quad \alpha=90.00^{\circ}$

$b=1397.4(2) \mathrm{pm} ; \quad \beta=91.48(3)^{\circ}$

$c=1691.7(3) \mathrm{pm} ; \quad \gamma=90.00^{\circ}$

$3.4662(10) \mathrm{nm}^{3}$

2

$2.404 \mathrm{Mg} / \mathrm{m}^{3}$

$12.642 \mathrm{~mm}^{-1}$

2328

$0.8 \times 0.6 \times 0.4 \mathrm{~mm}^{3}$

3.63 bis $25.01^{\circ}$

$-17 \leq h \leq 17,-15 \leq k \leq 16,-19 \leq l \leq 20$

7740

$6096\left(R_{\text {int }}=0.0402\right)$

6088 / 0 / 362

1.059

$R 1=0.0620, w R 2=0.1695$

$R 1=0.0688, w R 2=0.1824$

3411 und $-2864 \mathrm{e} \times \mathrm{nm}^{-3}$ 
Verbindung

Archivnummer

Summenformel

Molmasse

Temperatur

Wellenlänge

Kristallsystem

Raumgruppe

Zelldimensionen

Zellvolumen

Formeleinheiten pro Zelle $Z$

Berechnete Dichte

Absorptionskoeffizient

$F(000)$

Kristallgröße

Gemessener $\theta$-Bereich

Indexgrenzen

Anzahl gemessener Reflexe

Unabhängige Reflexe

Daten/Restraints/Parameter

Goodness-of-Fit an $F^{2}$

Endgültige $R$-Werte $[\mathrm{I}>2 \sigma(\mathrm{I})]$

$R$-Werte (sämtliche Daten)

Größtes Maximum und Minimum
26

roe 846

$\mathrm{C}_{34} \mathrm{H}_{51} \mathrm{~F}_{2} \mathrm{NSi}$

539.85

200.(2) K

$71.073 \mathrm{pm}$

Orthorhombisch

Pna2 1

$a=1839.9(3) \mathrm{pm} ; \quad \alpha=90.00^{\circ}$

$b=159.22(14) \mathrm{pm} ; \beta=90.00^{\circ}$

$c=1525.4(2) \mathrm{pm} ; \quad \gamma=90.00^{\circ}$

$3.2535(7) \mathrm{nm}^{3}$

4

$1.102 \mathrm{Mg} / \mathrm{m}^{3}$

$0.105 \mathrm{~mm}^{-1}$

1176

$1.00 \times 0.40 \times 0.10$

3.52 bis $25.04^{\circ}$

$-21 \leq h \leq 21,-2 \leq k \leq 13,-18 \leq l \leq 18$

3578

$3528\left(R_{\text {int }}=0.0099\right)$

$3522 / 1 / 347$

1.091

$R 1=0.0551, w R 2=0.1281$

$R 1=0.0816, w R 2=0.1610$

462 und $-299 \mathrm{e} \times \mathrm{nm}^{-3}$ 


\section{Literaturverzeichnis}

[1] F. Zabel, Chem. Unserer Zeit 1987, 21, 141.

[2] J. L. Spirlet, J. R. Peterson, L. B. Asprey, Adv. Inorg. Chem. 1987, 31 , 1.

[3] H. Millauer, W. Schwertfeger, G. Siegemund, Angew. Chem. 1985, 97, 164; Angew. Chem. Int. Ed. Engl. 1985, 24, 161.

[4] J. G. Riess, M. LeBlanc, Angew. Chem. 1978, 90, 654; Angew. Chem. Int. Ed. Engl. 1978, 17, 642.

[5] E. F. Murphy, R. Murugavel, H. W. Roesky, Chem. Rev. 1997, 97, 3425.

[6] H. Dorn, E. F. Murphy, S. A. A. Shah, H. W. Roesky, J. Fluorine. Chem. 1997, 86, 121.

[7] B. R. Jagirdar, E. F. Murphy, H. W. Roesky, Prog. Inorg. Chem. 1999, 48, 351.

[8] W. Kaminsky, S. Lenk, V. Scholz, H. W. Roesky, A. Herzog, Macromolecules, $1997,30,7647$.

[9] X. Verdaguer, U. E. W. Lange, M. T. Reding, S. L. Buchwald, J. Am. Chem. Soc. 1996, 118,6784 .

[10] J. L. Kiplinger, T. G. Richmond, J. Am. Chem. Soc. 1996, 118, 1805.

[11] F.-Q. Liu, A. Herzog, H. W. Roesky, I. Usón, Inorg. Chem. 1996, 35, 741.

[12] N. M. Doherty, N. W. Hoffman, Chem. Rev. 1991, 91, 553.

[13] N. W. Hoffman, N. Prokopuk, J. M. Robbins, J. C. Jones, N. M. Doherty, Inorg. Chem. 1991, 30, 4177.

[14] J. L. Kiplinger, T. G. Richmond, E. C. Osterberg, Chem. Rev. 1994, 94, 373.

[15] B. L. Lucht, M. J. Poss, M. A. King, T. G. Richmond, J. Chem. Soc., Chem. Commun. 1991, 400.

[16] A. C. Cooper, K. Folting, J. C. Huffman, K. G. Caulton, Organometallics 1997, 16, 505 .

[17] D. Viets, E. Lork, P. G. Watson, R. Mews, Angew. Chem. 1997, 109, 655; Angew. Chem. Int. Ed. Engl. 1997, 36, 623.

[18] W. Beck, K. Sünkel, Chem. Rev. 1988, 88, 1405.

[19] S. K. Agbossou, C. Roger, A. Igau, J. A. Gladysz, Inorg. Chem. 1992, 31, 419.

[20] D. Naumann, Fluor und Fluorverbindungen, 1. Aufl., Steinkopff, Darmstadt, 1980.

[21] A. Herzog, F.-Q. Liu, H. W. Roesky, A. Demsar, K. Keller, M. Noltemeyer, F. 
Pauer, Organometallics 1994, 13, 1251.

[22] H. W. Roesky, M. Sotoodeh, Y. Xu, F. Schrumpf, M. Noltemeyer, Z. Anorg. Allg. Chem. 1990, 580, 131.

[23] H. W. Roesky, F. Schrumpf, M. Noltemeyer, J. Chem. Soc., Dalton Trans. 1990, 713.

[24] G. Wilkinson, F. G. A. Stone, E. W. Abel, Comprehensive Organometallic Chemistry, 1. Auflage 1982, Vol. 3, Pergamon Press.

[25] M. Witt, H. W. Roesky, Prog. Inorg. Chem. 1992, 40, 353.

[26] P. G. Gassmann, W. H. Campbell, D. W. Macomber, Organometallics 1984, 3, 385.

[27] A. Antiñolo, M. F. Lappert, A. Singh, D. J. W. Winterborn, L. M. Engelhardt, C.

L. Raston, A. H. White, A. J. Carty, N. J. Taylor, J. Chem. Soc., Dalton Trans. 1987, 1463.

[28] P. M. Druce, B. M. Kingston, M. F. Lappert, T. R. Spalding, R. C. Srivastava, J. Chem. Soc. A 1969, 2106.

[29] H. W. Roesky, M. Sotoodeh, M. Noltemeyer, Angew. Chem. 1992, 104, 869; Angew. Chem. Int. Ed. Engl. 1992, 31, 864.

[30] A. Künzel, M. Sokolow, F.-Q. Liu, H. W. Roesky, M. Noltemeyer, H.-G. Schmidt, I Usón, J. Chem. Soc., Dalton Trans. 1996, 913.

[31] F.-Q. Liu, I. Usón, H. W. Roesky, J. Chem. Soc., Dalton Trans. 1995, 2453.

[32] P. Yu, E. F. Murphy, H. W. Roesky, P. Lubini, H.-G. Schmidt, M. Noltemeyer, Organometallics 1997, 16, 313.

[33] A. Herzog, H. W. Roesky, F. Jäger, A. Steiner, J. Chem. Soc., Chem. Commun. 1996, 29.

[34] C. Schnitter, K. S. Klimek, H. W. Roesky, T. Albers, H.-G. Schmidt, C. Röpken,

E. Parisini, Organometallics 1998, 17, 2249.

[35] F. A. Cotton, K. J. Wieser, Inorg. Chem. 1991, 31, 920.

[36] J. H. Shin, G. Parkin, Organometallics 1998, 17, 5689.

[37] L. Resconi, S. Bossi, L. Abis, Macromolecules 1990, 23, 4489.

[38] D. Cam, E. Albizzati, P. Cinquina, Macromol. Chem. 1990, 191, 1641.

[39] S. Pasynkiewicz, Polyhedron 1990, 9, 429.

[40] C. J. Harlan, M. R. Mason, A. R. Barron, Organometallics 1994, 13, 2957.

[41] A. K. Zefirova, A. E. Shilov, Dokl. Akad. Nauk SSSR 1961, 136, 599; Dokl. Chem. 
(Engl. Transl.) 1961, 136, 77.

[42] F. S. Dyachkovskii, A. K. Shilova, A. E. Shilov, J. Polym. Sci., C 1967, 16, 2333.

[43] A. Herzog, H. W. Roesky, Z. Zak, M. Noltemeyer, Angew. Chem. 1994, 106, 1035; Angew. Chem. Int. Ed. Engl. 1994, 33, 967.

[44] A. Herzog, H. W. Roesky, F. Jäger, A. Steiner, M. Noltemeyer, Organometallics 1996, 15, 909 .

[45] W. Uhl, Z. Naturforsch., Teil B 1988, 43, 1113.

[46] A. Sekiguchi, C. Kabuto, H. Sakurai, Angew. Chem. 1989, 101, 97; Angew. Chem., Int. Ed. Engl. 1989, 28, 55.

[47] H. J. Breunig, R. Rosler, E. Lork, Organometallics 1998, 17, 5594.

[48] H. J. Breunig, R. Rosler, E. Lork, Angew. Chem. 1998, 110, 3361; Angew. Chem., Int. Ed. 1998, 37, 3175.

[49] A. H. Cowley, J. E. Kilduff, J. G. Lasch, S. K. Mehrotra, N. C. Norman, M. Pakulski, B. R. Whittlesey, J. L. Atwood, W. E. Hunter, Inorg. Chem. 1984, 23, 2582 .

[50] A. H. Cowley, J. G. Lasch, N. C. Norman, M. Pakulski, J. Am. Chem. Soc., 1983, $105,5506$.

[51] M. Westerhausen, M. Hartmann, W. Schwarz, Inorg. Chim. Acta 1998, 269, 91.

[52] M. F. Lappert, P. I. Riley, P. I. W. Yarrow, J. L. Atwood, W. E. Hunter, M. J. Zaworotko, J. Chem. Soc., Dalton Trans. 1981, 814.

[53] G. K. Barker, M. F. Lappert, J. A. K. Howard, J. Chem. Soc., Dalton Trans. 1978, 734.

[54] M. Westerhausen, B. Rademacher, J. Organomet. Chem. 1991, 421, 175.

[55] C. K. Sperry, W. D. Cotter, R. A. Lee, R. J. Lachikotte, G. C. Bazan, J. Am. Chem. Soc. 1998, 120, 7791.

[56] Gmelins Handbuch der Anorganischen Chemie, Tantal Teil B, 8. Aufl., Verlag Chemie, Weinheim, 1970.

[57] H. Hao, H. W. Roesky, C. Cui, H.-G. Schmidt, M. Noltemeyer, P. Yu, G. Bai, Z. Anorg. Allg. Chem. 2000, 626, 368.

[58] M. Schormann, H. W. Roesky, M. Noltemeyer, H.-G. Schmidt, J. Fluorine Chem. 2000, 101,75 .

[59] R. R. Schrock, J. D. Fellman, J. Am. Chem. Soc. 1978, 100, 3359.

[60] O. I. Guzyr, M. Schormann, J. Schimkowiak, H. W. Roesky, C. Lehmann, M. G. 
Walawalkar, R. Murugavel, H.-G. Schmidt, M. Noltemeyer, Organometallics 1999, $18,832$.

[61] H. Schmidbaur, J. Weidlein, H.-F. Klein, K. Eiglmeier, Chem. Ber. 1968, 101, 2268.

[62] G. S. Kalinina, O. A. Kruglaya, B. I. Kubax, N. S. Vyazankin, Izv. Akad. Nauk SSSR, Ser. Khim. 1971, 9, 2102.

[63] M. J. S. Gynane, M. F. Lappert, S. J. Miles, A. J. Carty, N. J. Taylor, J. Chem. Soc., Dalton Trans. 1977, 2009.

[64] S. Weng, Acta Crystallogr. Sect. C 1995, 51, 2292.

[65] J. L. Lefferts, K. C. Molloy, M. B. Hossain, D. Van Der Helm, J. J. Zuckerman, J. Organomet. Chem. 1982, 240, 349.

[66] H. W. Roesky, Inorg. Chem. 1999, 38, 5934.

[67] A. Pevec, A. Demsar, V. Gramlich, S. Petricek, H. W. Roesky, J. Chem. Soc. 1997, 2215 .

[68] J. Sala-Pala, J. Amaudrut, J. E. Guerchais, R. Mercier, J. Douglade, J. G. Theobald, J. Organomet. Chem. 1981, 204, 347.

[69] R. J. Puddephatt, M. A. Stalteri, Organometallics 1983, 2, 1400.

[70] D. R. Neithamer, R. E. LaPointe, R. A. Wheeler, D. S. Richeson, G. D. Van Duyne, P. T. Wolczanski, J. Am. Chem. Soc. 1989, 111, 9056.

[71] R. L. Miller, R. Toreki, R. E. LaPointe, P. T. Wolczanski, G. D. Van Duyne, D. C. Roe, J. Am. Chem. Soc. 1993, 115, 5570.

[72] D. Labahn, Diplomarbeit, Göttingen 1989.

[73] O. Graalmann, U. Klingebiel, W. Clegg, M. Haase, G. M. Sheldrick, Angew. Chem. 1984, 96, 904; Angew. Chem. Int. Ed. Engl. 1984, 23, 891.

[74] K. Dippel, N. K. Keweloh, P. G. Jones, U. Klingebiel, D. Schmidt, Z. Naturforsch. Teil B 1987, 42, 1253.

[75] D. Schmidt-Bäse, U. Klingebiel, Chem. Ber. 1989, 122, 815.

[76] S. Schütte, C. Freire-Erdbrügger, U. Klingebiel, G. M. Sheldrick, Phosphorus, Sulfur, Silicon 1993, 78, 75.

[77] S. Schütte, U. Pieper, U. Klingebiel, D. Stalke, J. Organomet. Chem. 1993, 446, 45.

[78] P. B. Hitchcock, N. H. Buttrus, A. C. Sullivan, J. Organomet. Chem. 1986, 303, 321. 
[79] M. J. McGeary, K. Folting, W. E. Streib, J. C. Huffman, K. G. Caulton, Polyhedron, 1991, 10, 2699.

[80] G. Boche, A. Opel, M. Marsch, K. Harms, F. Haller, J. C. W. Lohrenz, C. Tümmler, W. Koch, Chem. Ber. 1992, 125, 2265.

[81] G. R. Fuentes, P. S. Coan, W. E. Streib, K. G. Caulton, Polyhedron 1991, 10, 2371 .

[82] F. Pauer, G. M. Sheldrick, Acta Crystallogr. Sect. C 1993, 49, 1283.

[83] O. V. Kononov, V. D. Lobkov, V. A. Igonin, S. V. Lindeman, V. E. Shklover, Y. T. Struchkov, Metalloorg. Khim. 1991, 4, 784.

[84] A. Mommertz, K. Dehnicke, J. Magull, Z. Naturforsch., Teil B 1996, 51, 1583.

[85] H. Friebolin, Ein- und zweidimensionale NMR-Spektroskopie, 2. Aufl., VCH Weinheim, 1992.

[86] R. G. Pearson, J. Am. Chem. Soc. 1963, 85, 3533.

[87] R. G. Pearson, Inorg. Chem. 1988, 27, 734.

[88] G. Wittig, K. Clauß, Liebigs Ann. Chem. 1952, 578, 136.

[89] A. Schmuck, K. Seppelt, Chem. Ber. 1989, 122, 803.

[90] T. Imori, T. D. Tilley, Polyhedron 1994, 13, 2231.

[91] E. F. Murphy, T. Lübben, A. Herzog, H. W. Roesky, A. Demsar, M. Noltemeyer, H.-G. Schmidt, Inorg. Chem. 1996, 35, 23.

[92] B. R. Jagirdar, E. F. Murphy, H. W. Roesky, Prog. Inorg. Chem. 1999, 48, 351.

[93] S. Dielkus, C. Drost, R. Herbst-Irmer, U. Klingebiel, Organometallics 1994, 13, 3985.

[94] D. Grosskopf, L. Marcus, U. Klingebiel, M. Noltemeyer, Phosphorus, Sulfur, Silicon 1994, 97, 113.

[95] E. F. Murphy, R. Murugavel, H. W. Roesky, M. Noltemeyer, H.-G. Schmidt, Z. Anorg. Allg. Chem. 1996, 622, 579.

[96] K.-H. Thiele, U. Böhme, K. Peters, E.-M. Peters, L. Walz, H. G. von Schnering, Z. Anorg. Allg. Chem. 1993, 619, 771.

[97] J. L. Atwood, G. K. Barker, J. Holton, W. E. Hunter, M. F. Lappert, R. Pearce, J. Am. Chem. Soc. 1977, 99, 6645.

[98] J. Sala-Pala, J. Y. Calves, J. E. Guerchais, S. Brownstein, J. C. Dewan, A. J. Edwards, Can. J. Chem. 1978, 56, 1545.

[99] R. Bock, Aufschlußmethoden der anorganischen und organischen Chemie, Verlag 
Chemie Weinheim, 1972.

[100] W. A. Szarek, G. W. Hay, B. Doboszewski, Carbohydr. Res. 1986, 155, 107.

[101] M. Bhattacharjee, M. K. Chaudhuri, J. Chem. Soc., Dalton Trans. 1987, 477.

[102] D. Landini, H. Molinari, M. Penso, A. Rampoldi, Synthesis 1988, 12, 953.

[103] P. Bosch, F. Camps, E. Chamorro, V. Gasol, A. Guerro, Tetrahedron Lett. 1987, $28,4733$.

[104] F. Camps, E. Chamorro, V. Gasol, A. Guerro, J. Org. Chem. 1989, 54, 4294.

[105] D. Albanese, D. Landini, M. Penso, Tetrahedron Lett. 1995, 36, 8865.

[106] J. J. Harland, J. S. Payne, R. O. Day, R. R. Holmes, Inorg. Chem. 1987, 26, 760.

[107] K. Tamao, T. Hayashi, Y. Ito, Organometallics 1992, 11, 2099.

[108] M. Gingras, Tetrahedron Lett. 1991, 32, 7381.

[109] A. S. Pilcher, L. A. Herman, P. DeShong, J. Am. Chem. Soc. 1995, 117, 5166.

[110] H. W. Roesky, A. Stasch, H. Hatop, C. Rennekamp, D. H. Hamilton, M. Noltemeyer, H.-G. Schmidt, Angew. Chem. 2000, 112, 177; Angew. Chem., Int Ed. 2000, 39, 171.

[111] K. Ziegler, E. Holzkamp, R. Köster, H. Lehmkuhl, Angew. Chem. 1955, 67, 213.

[112] K. Ziegler, R. Köster, Liebigs Ann. Chem. 1957, 608, 1.

[113] B. Neumüller, Coord. Chem. Rev. 1997, 158, 69.

[114] B. Neumüller, F. Gahlmann, Chem. Ber. 1993, 126, 1579.

[115] M. R. Kopp, T. Kräuter, B. Werner, B. Neumüller, Z. Anorg. Allg. Chem. 1998, $624,881$.

[116] M. R. Knopp, B. Neumüller, Z. Anorg. Allg. Chem. 1998, 624, 1393.

[117] D. Chakraborty, S. Horchler, R. Krätzner, S. P. Varkey, H. W. Roesky, I. Usón, M. Noltemeyer, H.-G. Schmidt, Inorg. Chem. 2000 (im Druck).

[118] G. Wedler, Lehrbuch der Physikalischen Chemie, 3. Aufl., VCH Weinheim, 1987.

[119] D. C. Bradley, R. C. Mehrotra, J. D. Swanwick, W. Wardlaw, J. Chem. Soc. 1953, 2025 .

[120] T. J. Boyle, T. M. Alam, E. R. Mechenbier, Inorg. Chem. 1997, 36, 3293.

[121] A. I. Yanovsky, F. M. Dolgushin, M. I. Yanovskaya, N. M. Kotova, Y. T. Struchkov, N. Y. Turova, Zh. Neorg. Khim. 1997, 42, 450.

[122] R. Schmid, A. Mosset, J. Galy, J. Chem. Soc., Dalton Trans. 1991, 1999.

[123] I. W. Forrest, A. P. Lane, Inorg. Chem. 1976, 15, 265.

[124] V. G. Kessler, N. Y. Turova, A. I. Yanovskii, A. I. Bilokon, Y. T. Struchkov, Zh. 
Neorg. Khim. 1991, 36, 1662.

[125] D. W. Hart, J. Schwartz, J. Am. Chem. Soc. 1974, 96, 8115.

[126] A. Herzog, Dissertation, Göttingen 1994.

[127] E. Horn, F. L. Wimmer, M. R. Snow, Inorg. Synth. 1989, 26, 81.

[128] E. Horn, M. R. Snow, Aust. J. Chem. 1984, 37, 35.

[129] A. Stasch, persönliche Mitteilung.

[130] Organikum, 16. Auflage, V. E. B. deutscher Verlag der Wissenschaften, Berlin, 1986.

[131] G. M. Sheldrick, SHELX-97, Programme zur Kristallstrukturverfeinerung, Universität Göttingen, 1997.

[132] M. Weidenbruch, H. Pesel, W. Peter, R. Steichen, J. Organomet. Chem. 1977, 141, 9.

[133] R. E. LaPointe, P. T. Wolczanski, G. D. Van Duyne, Organometallics, 1985, 4, 1810 .

[134] S. Moorhouse, G. Wilkinson, J. Chem. Soc., Dalton Trans. 1974, 2187.

[135] N. Wiberg, G. Wagner, G. Müller, J. Riede, J. Organomet. Chem., 1984, 271, 381.

[136] E. Krause, Ber. Dtsch. Chem. Ges. 1918, 51, 1447.

[137] H. Gilman, J. O. Robinson, J. Am. Chem. Soc. 1929, 51, 3112.

[138] F. Challenger, J. F. Wilkinson, J. Chem. Soc. 1922, 121, 91.

[139] G. Wittig, D. Hellwinkel, Chem. Ber. 1964, 97, 789.

[140] G. H. Llinás, M. Mena, F. Palacios, P. Royo, R. Serrano, J. Organomet. Chem. 1988, 340, 37.

[141] P. C. Wailes, H. Weigold, A. P. Bell, J. Organomet. Chem. 1972, 34, 155.

[142] R. D. Sanner, S. T. Carter, W. J. Bruton, J. Organomet. Chem. 1982, 240, 157.

[143] D. B. Morse, T. B. Rauchfuss, S. R. Wilson, J. Am. Chem. Soc. 1988, 110, 8234.

[144] R. C. Murray, L. Blum, A. H. Liu, R. R. Schrock, Organometallics 1985, 4, 953.

[145] N. Oshima, H. Suzuki, Y. Moro-oka, Chem. Lett. 1984, 7, 1161.

[146] W. A. Hermann, G. Brauer, N. Auner, U. Klingebiel, Synthetic Methods of Organometallic and Inorganic Chemistry, Georg Thieme, Stuttgart, New York, 1996.

[147] M. J. McCormick, R. A. Williams, L. J. Levine, T. P. Hanusa, Polyhedron, 1988, 7, 725 .

[148] H. Schmidbaur, F. Schindler, Chem. Ber. 1966, 99, 2178. 
Die vorliegende Arbeit wurde in der Zeit von Juli 1997 bis September 2000 unter Anleitung von Prof. Dr. Dr. h. c. mult. H. W. Roesky am Institut für Anorganische Chemie der Georg-August-Universität Göttingen angefertigt.

\section{Herrn Prof. Dr. Dr. h. c. mult. H. W. Roesky}

danke ich sehr herzlich für seine ständige Gesprächsbereitschaft, sein stetes Interesse am Fortgang der Arbeit sowie seine großzügige Unterstützung und seine freundliche Betreuung.

Bei den Damen und Herren der Spektroskopie- und Serviceabteilungen sowie allen weiteren Institutsangehörigen bedanke ich mich für die zuteil gewordene Unterstützung bei dieser Arbeit.

Allen Kolleginnen und Kollegen des Arbeitskreises danke ich für das gute Arbeitsklima und die gezeigte Hilfsbereitschaft. Besonderer Dank gebührt P. Böttcher, Dr. T. Borrmann, C. Cui, Dr. A. Gouzyr, H. Hao, S. Kiel, K. Klimek, Dr. N. Mösch-Zanetti, Dr. R. Siefken, Dr. U. Ritter und Dr. S. P. Varkey.

Mein Dank gilt auch Dr. M. Witt, K. Pahl, A. Stasch und M. Gorol für das Korrekturlesen dieser Arbeit.

Für die Durchführung der Einkristall-Röntgenstrukturanalysen danke ich den Herren Dr. M. Noltemeyer, H.-G. Schmidt und C. Lehmann. 


\section{Lebenslauf}

Mark Schormann

geboren am 06. Dezember 1969 in Bremen

Eltern Elvira Schormann, geb. Friedrichs

Harald Schormann

Staatsangehörigkeit

deutsch

Familienstand

verheiratet

\section{Schulausbildung}

$1976-1980$

Friedrich Ebert Schule Nienburg/Weser

$1980-1982$

Orientierungsstufe Nienburg/Weser

$1982-1989$

Hindenburgschule Nienburg/Weser

Mai 1989

Abitur

\section{Wehrdienst}

Juni 1989 - September 1990

Grundwehrdienst in Eckernförde, Flensburg und Kiel

\section{Hochschulbildung}

Oktober 1990

Studium der Chemie an der Georg-August-Universität Göttingen

Juli 1994

Diplomvorprüfung

September 1996 - Mai 1997

Diplomarbeit im Arbeitskreis von Prof. Dr. Dr h.c. mult. Roesky am Institut für Anorganische Chemie der Universität Göttingen

Juni 1997 - September 2000 Anfertigung der vorliegenden Dissertation unter Anleitung von Prof. Dr. Dr. h.c. mult. Roesky am Institut für Anorganische Chemie der Universität Göttingen 